JOURNAL OF THE

AMERICAN MATHEMATICAL SOCIETY

Volume 24, Number 1, January 2011, Pages 133-181

S 0894-0347(2010)00679-0

Article electronically published on September 23, 2010

\title{
SHEAVES ON AFFINE SCHUBERT VARIETIES, MODULAR REPRESENTATIONS, AND LUSZTIG'S CONJECTURE
}

\author{
PETER FIEBIG
}

\section{INTRODUCTION}

One of the fundamental problems in representation theory is the calculation of the simple characters of a given group. This problem often turns out to be difficult and there is an abundance of situations in which a solution is out of reach. In the case of algebraic groups over fields of positive characteristic we have a partial, but not yet a full answer.

In 1979, George Lusztig conjectured a formula for the simple characters of a reductive algebraic group defined over a field of characteristic greater than the associated Coxeter number; cf. [Lus80b]. Lusztig outlined in 1990 a program that led, in a combined effort of several authors, to a proof of the conjecture for almost all characteristics. This means that for a given root system $R$ there exists a number $N=N(R)$ such that the conjecture holds for all algebraic groups associated to the root system $R$ if the underlying field is of characteristic greater than $N$. This number, however, is unknown in all but low rank cases.

One of the essential steps in Lusztig's program was the construction of a functor between the category of intersection cohomology sheaves with complex coefficients on an affine flag manifold and the category of representations of a quantum group (this combines results of Kashiwara-Tanisaki, KT95, and Kazhdan-Lusztig, KL93]). This led to a proof of the quantum (i.e. characteristic 0) analog of the conjecture. Andersen, Jantzen and Soergel then showed that the characteristic zero case implies the characteristic $p$ case for almost all $p$ (cf. AJS94]).

One of the principal functors utilized in Lusztig's program was the affine version of the Beilinson-Bernstein localization functor. It amounts to realizing an affine Kac-Moody algebra inside the space of global differential operators on an affine flag manifold. A characteristic $p$ version of this functor is a fundamental ingredient in Bezrukavnikov's program for modular representation theory (cf. [BMR08]), and recently Frenkel and Gaitsgory used the Beilinson-Bernstein localization idea in order to study the critical level representations of an affine Kac-Moody algebra (cf. [FG06]).

There is, however, an alternative approach that links the geometry of an algebraic variety to representation theory. It was originally developed in the case of finite-dimensional complex simple Lie algebras by Soergel (cf. [Soe90]). The idea was to give a "combinatorial description" of both the topological and the

Received by the editors June 24, 2008 and, in revised form, November 26, 2009, and July 16, 2010.

2010 Mathematics Subject Classification. Primary 20C20; Secondary 55N30.

(C)2010 American Mathematical Society Reverts to public domain 28 years from publication 
representation-theoretic categories in terms of the underlying root system using Jantzen's translation functors. This approach gives a new proof of the KazhdanLusztig conjecture, but it is also important in its own right: when taken together with the Beilinson-Bernstein localization it establishes the celebrated Koszul duality for simple finite-dimensional complex Lie algebras (cf. [Soe90, BGS96]).

In this paper we develop the combinatorial approach for quantum and modular representations. We relate a certain category of sheaves of $k$-vector spaces on an affine flag manifold to representations of the $k$-Lie algebra or the quantum group associated to Langlands' dual root datum (the occurrence of Langlands' duality is typical for this type of approach). As a corollary we obtain Lusztig's conjecture for quantum groups and for modular representations for large enough characteristics.

The main tool that we use is the theory of sheaves on moment graphs, which originally appeared in the work on the localization theorem for equivariant sheaves on topological spaces by Goresky, Kottwitz and MacPherson (cf. [GKM98]) and Braden and MacPherson (cf. [BM01]). In particular, we state a conjecture in terms of moment graphs that implies Lusztig's quantum and modular conjectures for all relevant characteristics.

Although there is no general proof of this moment graph conjecture yet, some important instances are known: The smooth locus of a moment graph is determined in Fie06, which yields the multiplicity-one case of Lusztig's conjecture in full generality. Moreover, by developing a Lefschetz theory on a moment graph we obtain in Fie08c an upper bound on the exceptional primes, i.e. an upper bound for the number $N$ referred to above. Although this bound is huge (in particular, much greater than the Coxeter number), it can be calculated by an explicit formula in terms of the underlying root system.

In the remainder of this introduction we explain our approach and the results in more detail.

1.1. The basic data. Let $G=G_{\mathbb{C}}$ be a connected, simply connected complex algebraic group. We fix a Borel subgroup $B \subset G$ and a maximal torus $T \subset B$. Let $X=\operatorname{Hom}\left(T, \mathbb{C}^{\times}\right)$be the character lattice of $T$ and $R^{+} \subset R \subset X$ the sets of roots of $B$ and of $G$. We denote by $X^{\vee}=\operatorname{Hom}\left(\mathbb{C}^{\times}, T\right)$ the cocharacter lattice, and by $R^{\vee} \subset X^{\vee}$ the dual root system. We let $\mathcal{W} \subset \mathrm{GL}\left(X^{\vee}\right)$ be the Weyl group, $\widehat{\mathcal{W}}=\mathcal{W} \ltimes \mathbb{Z} R^{\vee} \subset \operatorname{Aff}\left(X^{\vee}\right)$ the affine Weyl group and $h$ the Coxeter number of our data, i.e. the height of the highest root +1 . By $\widehat{\mathcal{S}} \subset \widehat{\mathcal{W}}$ we denote the set of simple affine reflections.

We fix an algebraically closed field $k$ of characteristic $p>h$ and let $G^{\vee}=G_{k}^{\vee}$ be the connnected simple algebraic group over $k$ that is the Langlands dual of $G$. We let $T^{\vee} \subset G^{\vee}$ be a maximal torus and identify $\operatorname{Hom}\left(T^{\vee}, \mathbb{C}^{\times}\right)$with the cocharacter lattice $X^{\vee}$. We let $\mathfrak{g}^{\vee}$ and $\mathfrak{h}^{\vee}$ be the Lie algebras of $G^{\vee}$ and $T^{\vee}$.

1.2. Multiplicities of modular representations. It is known how to calculate the characters of the simple rational representations of the reductive algebraic groups with root system $R^{\vee}$ from the characters of simple objects in a certain category $\mathcal{C}$ of restricted representations of $\mathfrak{g}^{\vee}$ that carry an additional action of $T^{\vee}$ (i.e., an $X^{\vee}$-grading), such that $\mathfrak{h}^{\vee} \subset \mathfrak{g}^{\vee}$ acts via the differential of the $T^{\vee}$-action. The simple objects in $\mathcal{C}$ are parametrized by their highest weights, so for $\lambda \in X^{\vee}$ denote by $L(\lambda)$ the corresponding simple object. It can be constructed as the unique 
simple quotient of the standard (or baby Verma) module $Z(\lambda)$ with highest weight $\lambda$.

The characters of the standard modules are easy to compute, and in order to get the characters of the simple modules it is enough to know the number $[Z(\lambda): L(\mu)]$ of occurrences of $L(\mu)$ as a subquotient in a Jordan-Hölder filtration of $Z(\lambda)$ for all $\lambda, \mu \in X^{\vee}$. From the linkage and translation principles it follows that it is sufficient to consider the simple and standard modules in the principal block of $\mathcal{C}$, i.e. those objects which correspond to weights of the form $\lambda=x \cdot{ }_{p} 0$ and $\mu=y \cdot{ }_{p} 0$ for $x, y \in \widehat{\mathcal{W}}$ (here ".p" denotes a shifted and elongated action of $\widehat{\mathcal{W}}$ on $X^{\vee}$ ).

We identify the set $\widehat{\mathcal{W}}$ with the set $\mathscr{A}$ of alcoves for the affine action of $\widehat{\mathcal{W}}$ on $X^{\vee} \otimes_{\mathbb{Z}} \mathbb{R}$ by fixing a base alcove $A_{e}$ (so that $w$ corresponds to the alcove $w . A_{e}$ ). In Soe97] a polynomial $p_{A, B}$ is associated to each pair of alcoves (following [Lus80a]).

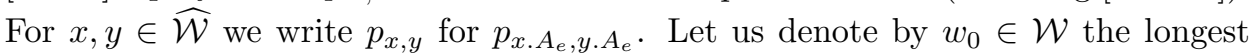
element in the finite Weyl group.

Conjecture. Suppose that char $k>h$. For $x, y \in \widehat{\mathcal{W}}$ we have

$$
\left[Z\left(x \cdot{ }_{p} 0\right): L\left(y \cdot{ }_{p} 0\right)\right]=p_{w_{0} x, w_{0} y}(1) .
$$

The above conjecture is known as the generic Lusztig conjecture. In [Fie07] we prove the (well-known) fact that it is equivalent to Lusztig's original conjecture on the characters of simple rational representations (cf. [Lus80b]).

Using an inherent symmetry one can show that it is enough to verify this conjecture in the case that $y$ is an element in the "anti-fundamental box", i.e. in the case that $y$ satisfies $-p<\left\langle\alpha, y \cdot{ }_{p} 0\right\rangle<0$ for each simple root $\alpha$. Denote by $\widehat{\mathcal{W}}^{r e s},-\subset \widehat{\mathcal{W}}$ the set of such elements.

1.3. The quantum analog. Let us just quickly state, without giving details, that there is a characteristic 0 analog of the representation theory described above (cf. the overview articles And95 and Soe95). Lusztig associated to the root system $R$, its set of positive roots $R^{+} \subset R$, an integer $l$ prime to all entries of the Cartan matrix, and a primitive $l$-th root of unity $\zeta$ the restricted quantum group $\mathbf{u}$, which is a finite-dimensional $\mathbb{Z} R$-graded algebra over $k=\mathbb{Q}(\zeta)$. For each $\lambda \in X$ one constructs a standard object $Z_{q}(\lambda)$ and a simple object $L_{q}(\lambda)$ in the category of $\mathbb{Z} R$-graded $\mathbf{u}$-modules and one can state a conjecture in complete analogy to the conjecture above. Let us refer to these two conjectures as Lusztig's modular conjecture and Lusztig's quantum conjecture.

1.4. Results. The main application of the relations that we construct between sheaves on affine Schubert varieties, sheaves on the underlying moment graph and representation theory is a proof of the following theorem.

Theorem 1.1. (1) Lusztig's quantum conjecture holds.

(2) The multiplicity-one case of Lusztig's modular conjecture holds, i.e. for all fields $k$ with char $k=p>h$ we have $\left[Z\left(x \cdot{ }_{p} 0\right): L\left(y \cdot{ }_{p} 0\right)\right]=1$ if and only if $p_{w_{0} x, w_{0} y}(1)=1$.

(3) There is an explicit number $U\left(\widehat{w}_{0}\right)$, defined in terms of the root system, such that Lusztig's modular conjecture holds for all fields $k$ with char $k>U\left(\widehat{w}_{0}\right)$.

Part (1) of the above theorem has already been proven by combining results of Kashiwara-Tanisaki and Kazhdan-Lusztig which form major steps in Lusztig's program. Our proof is independent and uses only the combinatorial description of the 
quantum category given by Andersen, Jantzen and Soergel. Part (2) is an application of the result on the "smooth locus" of a moment graph given in [Fie06]. Part (3) finally uses the main result in [Fie08c], where we develop a Lefschetz theory on a moment graph in order to calculate the number $U\left(\widehat{w}_{0}\right)$.

In the remainder of the introduction we want to describe the main ideas in the proofs of the above results.

1.5. Deformed representation theory. Each simple object $L(\mu)$ admits a projective cover $P(\mu)$ in $\mathcal{C}$. Moreover, each $P(\mu)$ has a finite filtration with subquotients that are isomorphic to various $Z(\lambda)$. The corresponding multiplicity, denoted by $(P(\mu): Z(\lambda))$, is independent of the particular filtration, and the following Brauertype reciprocity formula was shown by Humphreys (cf. [Hum71]):

$$
[Z(\lambda): L(\mu)]=(P(\mu): Z(\lambda)) .
$$

Let $S=S\left(\mathfrak{h}^{\vee}\right)$ be the symmetric algebra of $\mathfrak{h}^{\vee}$, and denote by $\tilde{S}$ the completion of $S$ at the maximal ideal generated by $\mathfrak{h}^{\vee}$. In order to compute the above multiplicities, Andersen, Jantzen and Soergel construct in AJS94 deformed versions $\widetilde{P}(\mu)$ and $\widetilde{Z}(\lambda)$ of $P(\mu)$ and $Z(\lambda)$, which appear in a deformed version $\widetilde{\mathcal{C}}$ of $\mathcal{C}$. Again, each $\widetilde{P}(\mu)$ has a finite filtration with subquotients isomorphic to various $\widetilde{Z}(\lambda)$, and for the multiplicities we have

$$
(\widetilde{P}(\mu): \widetilde{Z}(\lambda))=(P(\mu): Z(\lambda)) .
$$

The objects $\widetilde{P}(y \cdot p 0)$ can be constructed using translation functors: to each simple affine reflection $s \in \widehat{\mathcal{S}}$ one associates a translation functor $\theta^{s}$ on the principal block of $\widetilde{\mathcal{C}}$. We show that for each $y \in \widehat{\mathcal{W}}^{r e s,-}$ the object $\widetilde{P}\left(y \cdot{ }_{p} 0\right)$ appears as a direct summand in $\theta^{t} \circ \cdots \circ \theta^{s} \widetilde{Z}(0)$ for a suitable sequence $s, \ldots, t \in \widehat{\mathcal{S}}$. This serves as a motivation to define $\mathcal{R}$ as the smallest full subcategory of $\widetilde{\mathcal{C}}$ that is stable under taking direct sums and direct summands, contains $\widetilde{Z}(0)$ and with each object $M$ and each $s \in \widehat{\mathcal{S}}$ the object $\theta^{s} M$.

Let us just mention that in the quantum case we find analogous structures and results and, in particular, we have a category $\mathcal{R}$ over the field $k=\mathbb{Q}(\zeta)$ that encodes the structure of the quantum category.

In the following we relate the category $\mathcal{R}$ to equivariant sheaves of $k$-vector spaces on a complex affine flag variety.

1.6. Equivariant sheaves. Denote by $G((t))$ the loop group associated to $G$, and by $I \subset G((t))$ the Iwahori subgroup corresponding to $B$. The quotient $\widehat{\mathcal{F} 1}=$ $G((t)) / I$ carries a natural complex ind-variety structure and is called the affine flag variety. Denote by $\widehat{T}=T \times \mathbb{C}^{\times}$the extended torus and let it act on $G((t))$ such that the first factor acts by left multiplication and the second by rotating the loops. Then $\widehat{T}$ also acts on $\widehat{\mathcal{F l}}$.

Now let $k$ be a field of characteristic $\neq 2$ and let $\mathrm{D}_{\widehat{T}}(\widehat{\mathcal{F}} 1, k)$ be the bounded equivariant derived category of sheaves of $k$-vector spaces on $\widehat{\mathcal{F}}$. We define the category $\mathcal{I}$ of special equivariant sheaves on $\widehat{\mathcal{F}}$ following Soe00]. It is the smallest full subcategory of $\mathrm{D}_{\widehat{T}}(\widehat{\mathcal{F l}}, k)$ that is stable under taking direct sums and direct summands and under shifting, contains the constant equivariant sheaf $F_{e}$ of rank one on the base point of $\widehat{\mathcal{F}}$, and with each $F$ and each $s \in \widehat{\mathcal{S}}$ the sheaf $\pi_{s}^{*} \pi_{s *} F$ in $\mathrm{D}_{\widehat{T}}(\widehat{\mathcal{F} 1}, k)$ (here $\pi_{s}: \widehat{\mathcal{F} 1} \rightarrow \widehat{\mathcal{F l}}_{s}$ is the canonical map onto the partial affine flag 
variety corresponding to $s$ ). In [FW] we give another, more intrinsic definition of the category $\mathcal{I}$ as the category of certain equivariant parity sheaves on $\widehat{\mathcal{F}}$.

The set of $\widehat{T}$-fixed points in $\widehat{\mathcal{F}} 1$ is discrete and can canonically be identified with the set $\widehat{\mathcal{W}}$. So for $x \in \widehat{\mathcal{W}}$ we denote by $i_{x}:\{p t\} \rightarrow \widehat{\mathcal{F} 1}$ the corresponding inclusion. The following result is the most important step in the proof of Theorem 1.1.

Theorem 1.2. Suppose $k=\mathbb{Q}(\zeta)$ or that $k$ is of characteristic $p>h$.

(1) There exists an additive functor

$$
\Phi: \mathcal{I} \rightarrow \mathcal{R}
$$

such that $\Phi\left(F_{e}\right) \cong \widetilde{Z}(0)$ and such that $\Phi\left(\pi_{s}^{*} \pi_{s *} F\right) \cong \theta^{s} \Phi(F)$ for all $s \in \widehat{\mathcal{S}}$ and $F \in \mathcal{I}$.

(2) Each $\Phi(F)$ has a filtration by deformed standard modules, and the ranks of the local equivariant hypercohomologies of $F$ on $\widehat{T}$-fixed points yield multiplicities for $\Phi(F)$; i.e., we have

$$
\operatorname{rk} \mathbb{H}_{\widehat{T}}^{*}\left(i_{x}^{*} F\right)=\left(\Phi(F): \widetilde{Z}\left(x \cdot{ }_{p} 0\right)\right)
$$

for all $x \in \widehat{\mathcal{W}}$.

(Here, "rk" refers to the rank of a free $H_{\widehat{T}}^{*}(p t, k)$-module.) Now we explain how to derive part (1) of Theorem 1.1 from the statement above.

1.7. Intersection cohomology sheaves. We identify the set of $I$-orbits in $\widehat{\mathcal{F}} 1$ with the affine Weyl group, so we denote by $\mathcal{O}_{y} \subset \widehat{\mathcal{F}}$ the orbit corresponding to $y \in \widehat{\mathcal{W}}$ and let $\mathrm{IC}_{\widehat{T}, y} \in \mathrm{D}_{\widehat{T}}(\widehat{\mathcal{F} l}, k)$ be the $\widehat{T}$-equivariant intersection cohomology complex on the Schubert variety $\overline{\mathcal{O}_{y}}$ with coefficients in $k$.

If $k$ is a field of characteristic zero, then the decomposition theorem and some orbit combinatorics show that $\mathcal{I}$ is the category of direct sums of shifted $\widehat{T}$-equivariant intersection cohomology sheaves on Schubert varieties in $\widehat{\mathcal{F}}$. The equivariant analogue of a result of Kazhdan and Lusztig in [KL79] is that we have rk $\mathbb{H}_{\widehat{T}}^{*}\left(i_{x}^{*} \mathrm{IC}_{\widehat{T}, y}\right)=$ $h_{x, y}(1)$ for all $x, y \in \widehat{\mathcal{W}}$, where $h_{x, y}$ is an affine Kazhdan-Lusztig polynomial (cf. Theorem 4.4).

Now fix an arbitrary sequence $s, \ldots, t \in \widehat{\mathcal{S}}$. The object $\pi_{t}^{*} \pi_{t *} \cdots \pi_{s}^{*} \pi_{s *} F_{e}$ can be defined over $\mathbb{Z}$ and it decomposes in almost all characteristics as it does in characteristic zero. We deduce that $\pi_{t}^{*} \pi_{t *} \cdots \pi_{s}^{*} \pi_{s *} F_{e}$ is a direct sum of shifted intersection cohomology sheaves on Schubert varieties for all fields $k$ of big enough characteristic (the notion of big enough now depends on the chosen sequence).

Denote by $\widehat{w}_{0} \in \widehat{\mathcal{W}}$ the largest element in $\widehat{\mathcal{W}}^{\text {res, }}$ - (with respect to the Bruhat order), and set $\widehat{\mathcal{W}}^{\circ}=\left\{w \in \widehat{\mathcal{W}} \mid w \leq \widehat{w}_{0}\right\}$. Define $\mathcal{I}^{\circ} \subset \mathcal{I}$ as the full subcategory that consists of direct sums of shifted direct summands of the objects $\pi_{t}^{*} \pi_{t *} \cdots \pi_{s}^{*} \pi_{s *} F_{e}$, where $s \cdots t$ is a reduced expression of an element in $\widehat{\mathcal{W}}^{\circ}$. Since for the construction of $\mathcal{I}^{\circ}$ we have to consider only finitely many sequences, we can deduce the following:

Theorem 1.3. Suppose that $k$ is a field of characteristic 0 or $p \gg 0$.

(1) $\mathcal{I}^{\circ}$ is the full subcategory of $D_{\widehat{T}}(\widehat{\mathcal{F} 1}, k)$ that consists of objects isomorphic to a direct sum of shifted intersection cohomology sheaves $\mathrm{IC}_{\widehat{T}, y}$ with $y \in \widehat{\mathcal{W}}^{\circ}$. 
(2) If $y \in \widehat{\mathcal{W}}^{\circ}$ and $y=s \cdots t$ is a reduced expression, then $\mathrm{IC}_{\widehat{T}, y}$ occurs as the unique indecomposable direct summand in $\pi_{t}^{*} \pi_{t *} \cdots \pi_{s}^{*} \pi_{s *} F_{e}$ that is supported on $\overline{\mathcal{O}_{y}}$.

(3) For $y \in \widehat{\mathcal{W}}^{\circ}$ and $x \in \widehat{\mathcal{W}}$ we have $\operatorname{rk} \mathbb{H}_{\widehat{T}}^{*}\left(i_{x}^{*} \mathrm{IC}_{\widehat{T}, y}\right)=h_{x, y}(1)$.

Again one hopes that each prime above the Coxeter number is big enough for the statements of the theorem to hold.

In Section 8 we show that we have $\Phi\left(\mathrm{IC}_{\widehat{T}, y}\right) \cong \widetilde{P}\left(y \cdot_{p} 0\right)$ under the assumption of Theorem 1.3. Moreover, the following mysterious relation between the KazhdanLusztig polynomials $h$ and the periodic polynomial $p$ holds. Although $h_{x, y} \neq$ $p_{w_{0} x, w_{0} y}$ in general, we have $h_{x, y}(1)=p_{w_{0} x, w_{0} y}(1)$ for all $y \in \widehat{\mathcal{W}}^{r e s,-}$ and all $x \in \widehat{\mathcal{W}}$ (this phenomenon is explained by the fact that the functor $\Psi$ defined below does not preserve gradings). So part (1) of Theorem 1.1 is a consequence of Theorem 1.2 and Theorem 1.3. In addition, we can already deduce Lusztig's modular conjecture for almost all primes. Let us now discuss the main steps of the definition $\Phi$.

1.8. Categorifications of combinatorial data. Let $\mathbf{H}$ be the affine Hecke algebra associated to $R$ and let $\mathbf{M}$ be its periodic module (cf. [Soe97]). Denote by $A_{e}$ the basis element in $\mathbf{M}$ corresponding to the fundamental alcove, and consider the map $\psi: \mathbf{H} \rightarrow \mathbf{M}$ that is obtained by letting $\mathbf{H}$ act on $A_{e}$.

For each field $k$ with characteristic different from 2 and different from 3 if $R$ is of type $G_{2}$ we construct categories $\mathcal{H}$ and $\mathcal{M}$ and a functor $\Psi: \mathcal{H} \rightarrow \mathcal{M}$ together with character maps $h_{\mathcal{H}}: \mathcal{H} \rightarrow \mathbf{H}$ and $h_{\mathcal{M}}: \mathcal{M} \rightarrow \mathbf{M}$ (i.e. maps to the Grothendieck groups), such that the diagram

commutes.

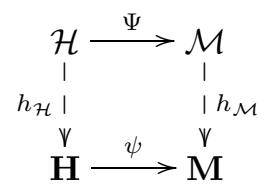

The category $\mathcal{H}$ is a full subcategory of the category of modules over the equivariant cohomology of $\widehat{\mathcal{F l}}$ (with coefficients in $k$ ), and hypercohomology $\mathbb{H}_{\widehat{T}}^{*}$ yields a functor from $\mathcal{I}$ to $\mathcal{H}$. The category $\mathcal{M}$ appears in the work of Andersen, Jantzen and Soergel. There an equivalence $\mathbb{V}$ between $\mathcal{R}$ and an $\tilde{S}$-linear version $\widetilde{\mathcal{M}}$ of $\mathcal{M}$ is constructed in the case that $k$ is an algebraically closed field of characteristic $p>h$. Our functor is then the composition

$$
\Phi: \mathcal{I} \stackrel{\mathbb{H}_{\widehat{T}}^{*}}{\longrightarrow} \mathcal{H} \stackrel{\Psi}{\longrightarrow} \mathcal{M} \stackrel{\cdot \otimes_{S} \tilde{S}}{\longrightarrow} \widetilde{\mathcal{M}} \stackrel{\mathbb{V}^{-1}}{\longrightarrow} \mathcal{R} .
$$

We now relate $\mathcal{H}$ to yet another category.

1.9. Sheaves on moment graphs. In the papers [Fie06] and Fie08c we study the theory of sheaves on moment graphs, which gives the following alternative description of the indecomposable objects in $\mathcal{H}$ (cf. [Fie08b]).

To the action of $\widehat{T}$ on $\widehat{\mathcal{F l}}$ one can associate a moment graph over the lattice $\widehat{X}=\operatorname{Hom}\left(\widehat{T}, \mathbb{C}^{\times}\right)$that plays a prominent role in equivariant algebraic topology (cf. GKM98, BM01]). Recall that an (ordered) moment graph over a lattice $Y$ is a graph together with a partial order on its set of vertices and a map that associates to each edge a nonzero element in $Y$. 
In our situation such a moment graph $\widehat{\mathcal{G}}$ is obtained as follows. Its vertices are the $\widehat{T}$-fixed point in $\widehat{\mathcal{F}}$ and its edges are the one-dimensional $\widehat{T}$-orbits (the closure of a one-dimensional orbit contains exactly two fixed points). Each edge is labelled by the positive affine root associated to the rotation action of $\widehat{T}$ on the corresponding orbit. The partial order is induced by the closure relation on the Iwahori-orbits in $\widehat{\mathcal{F}} \mathrm{l}$ (each such orbit contains a unique $\widehat{T}$-fixed point). For the applications in this article, only the full subgraph $\widehat{\mathcal{G}}^{\circ} \subset \widehat{\mathcal{G}}$ that consists of the vertices corresponding to elements in $\widehat{\mathcal{W}}^{\circ}$ plays a role. It has the advantage of being finite.

Suppose that $k$ is a field of characteristic $\neq 2$ and set $\widehat{V}_{k}:=\widehat{X} \otimes_{\mathbb{Z}} k$. To $\widehat{\mathcal{G}}^{\circ}$ one can associate a category of $k$-sheaves on $\widehat{\mathcal{G}}^{\circ}$, and to each vertex $y$ one assigns the intersection (or canonical) sheaf $\mathscr{B}_{y}$. Denote by $\mathscr{B}_{y}^{x}$ its stalk at the vertex $x$. It is a graded free module over the symmetric algebra $\widehat{S}:=S\left(\widehat{V}_{k}\right)$.

Now the restriction on the characteristic of $k$ is as follows. A large part of the theory of $k$-sheaves on a moment graph $\mathcal{G}$ behaves well only in the case that the pair $(\mathcal{G}, k)$ satisfies the GKM-property, which means that the labels on two different edges meeting at a common vertex are linearly independent over $k$ (cf. [Fie08a]). We will show that for $\widehat{\mathcal{G}}^{\circ}$ this is the case if char $k$ is at least the Coxeter number.

We define a certain subcategory $\mathcal{H}^{\circ}$ of $\mathcal{H}$ that contains all relevant objects. From the results in [Fie08b] we deduce that for char $k \geq h$ the category $\mathcal{H}^{\circ}$ can be interpreted as the category of direct sums of the spaces of global sections of the intersection sheaves $\mathscr{B}_{y}$ on $\widehat{\mathcal{G}}^{\circ}$. This, together with the functor $\Psi$ and the Andersen-Jantzen-Soergel equivalence, gives us a way to link the moment graph theory to modular representation theory, and in Section 9.3 we state a conjecture that implies Lusztig's conjecture. The main results in [Fie06] and Fie08c prove certain instances of this conjecture and yield part (2) and part (3) of Theorem 1.1

1.10. Contents. In Section 2 we discuss the affinization of a root system and define an algebra $\widehat{\mathcal{Z}}$ over the subring $\mathbb{Z}^{\prime}=\mathbb{Z}[1 / 2]$ of $\mathbb{Q}$. For each simple affine reflection we construct a translation functor on the category of $\widehat{\mathcal{Z}}$-modules, and we define the category $\mathcal{H}$ of special $\widehat{\mathcal{Z}}$-modules as the full subcategory generated from a unit object by repeatedly applying translation functors.

In Section 3 we discuss various localizations of special modules. This is used in Section 4 to define a functorial filtration on the category $\mathcal{H}$ for each partial order on the set $\widehat{\mathcal{W}}$ that is compatible with the involutions given by the simple affine reflections. To such a filtration corresponds a character map from $\mathcal{H}$ to the free $\mathbb{Z}\left[v, v^{-1}\right]$-module with basis $\widehat{\mathcal{W}}$. It turns out that the Bruhat order naturally yields characters in the affine Hecke algebra, whereas the generic Bruhat order yields characters in its periodic module.

In Section 5 we recall the definition of the category $\mathcal{M}$ introduced by Andersen, Jantzen and Soergel. In analogy to the definition of $\mathcal{H}$, also $\mathcal{M}$ is generated inside a certain category $\mathcal{K}$ by repeatedly applying translation functors to a unit object. This allows us to define the functor $\Psi: \mathcal{H} \rightarrow \mathcal{M}$. Although the functor itself is easy to define, it is quite tedious to check that it intertwines the translation functors.

The definition of translation functors on $\mathcal{K}$ that we use is different from the definition of Andersen, Jantzen and Soergel (it is a version "without constants"). In Section 6 we show that both definitions lead to equivalent categories. 
In Section 7 we define the category $\mathcal{I}$ of special sheaves on the affine flag variety and show that the hypercohomology functor can be considered as a functor from $\mathcal{I}$ to $\mathcal{H}$. We use the decomposition theorem to deduce multiplicity formulas for the objects in $\mathcal{H}$ for almost all characteristics.

In Section 8 we recall the main result of AJS94, which relates the category $\mathcal{M}$ to the category $\mathcal{R}$ of representations of a Lie algebra or a quantum group. The multiplicity formulas that we gained in Section 7 for $\mathcal{H}$, together with the functor $\Psi: \mathcal{H} \rightarrow \mathcal{M}$, give multiplicity formulas for objects in $\mathcal{R}$.

We interpret our main category $\mathcal{H}$ as a category of sheaves on moment graphs in Section 9, and state a conjecture on the multiplicities of stalks of the intersection sheaves on the graph. In [Fie06] and [Fie08c] two instances of this conjecture are proven. In a last step we apply this to Lusztig's conjecture.

\section{A CATEgory associated to A RoOT System}

Let $V$ be a finite-dimensional $\mathbb{Q}$-vector space, let $V^{*}=\operatorname{Hom}_{\mathbb{Q}}(V, \mathbb{Q})$ be its dual space and denote by $\langle\cdot, \cdot\rangle: V \times V^{*} \rightarrow \mathbb{Q}$ the canonical pairing. Let $R \subset V$ be a reduced and irreducible root system. For a root $\alpha \in R$ denote by $\alpha^{\vee} \in V^{*}$ its coroot. Let $R^{\vee}=\left\{\alpha^{\vee} \mid \alpha \in R\right\}$ be the coroot system.

Denote by $s_{\alpha} \in \operatorname{GL}\left(V^{*}\right)$ the reflection associated to $\alpha \in R$, i.e. the linear map given by $s_{\alpha}(v)=v-\langle\alpha, v\rangle \alpha^{\vee}$. Let $\mathcal{W} \subset \mathrm{GL}\left(V^{*}\right)$ be the Weyl group, i.e. the subgroup generated by the $s_{\alpha}$ with $\alpha \in R$.

2.1. The affine Weyl group. For $\alpha \in R$ and $n \in \mathbb{Z}$ define the affine hyperplane

$$
H_{\alpha, n}:=\left\{v \in V^{*} \mid\langle\alpha, v\rangle=n\right\} .
$$

We denote by $s_{\alpha, n}$ the reflection at $H_{\alpha, n}$, i.e. the affine transformation on $V^{*}$ that maps $v \in V^{*}$ to

$$
s_{\alpha, n}(v):=v-(\langle\alpha, v\rangle-n) \alpha^{\vee} .
$$

The affine Weyl group $\widehat{\mathcal{W}} \subset \operatorname{Aff}\left(V^{*}\right)$ is the group of affine transformations on $V^{*}$ that is generated by the set $\widehat{\mathcal{T}}$ of all reflections $s_{\alpha, n}$ with $\alpha \in R$ and $n \in \mathbb{Z}$.

Since $s_{\alpha}=s_{\alpha, 0}$, the finite Weyl group $\mathcal{W}$ appears as a subgroup of $\widehat{\mathcal{W}}$. Let $\mathbb{Z} R^{\vee} \subset$ $V^{*}$ be the coroot lattice. To $x \in \mathbb{Z} R^{\vee}$ we associate the translation $t_{x}: V^{*} \rightarrow V^{*}$, $v \mapsto v+x$. For $\alpha^{\vee} \in R^{\vee}$ we have $t_{\alpha^{\vee}}=s_{\alpha, 1} \circ s_{\alpha, 0}$; hence $\widehat{\mathcal{W}}$ contains the abelian group $\mathbb{Z} R^{\vee}$. We even have $\widehat{\mathcal{W}}=\mathcal{W} \ltimes \mathbb{Z} R^{\vee}$ and we denote by $:$ : $\widehat{\mathcal{W}} \rightarrow \mathcal{W}, w \mapsto \bar{w}$, the corresponding quotient map. Then $\overline{s_{\alpha, n}}=s_{\alpha}$.

2.2. A linearization. Set $\widehat{V}:=V \oplus \mathbb{Q}$, so $\widehat{V}^{*}=V^{*} \oplus \mathbb{Q}$, and define for $\alpha \in R$ and $n \in \mathbb{Z}$ a linear action of $s_{\alpha, n}$ on $\widehat{V}^{*}$ by

$$
s_{\alpha, n}(v, \mu):=\left(v-(\langle\alpha, v\rangle-\mu n) \alpha^{\vee}, \mu\right) .
$$

This extends to a linear action of $\widehat{\mathcal{W}}$ on $\widehat{V}^{*}$ which leaves the level spaces $V_{\kappa}^{*}=$ $\left\{(v, \kappa) \mid v \in V^{*}\right\} \cong V^{*}$ for $\kappa \in \mathbb{Q}$ stable. On $V_{1}^{*}$ we recover the affine action of $\widehat{\mathcal{W}}$ and on $V_{0}^{*}$ the affine Weyl group $\widehat{\mathcal{W}}$ acts via the ordinary action of its finite quotient $\mathcal{W}$.

The hyperplane in $\widehat{V}^{*}$ fixed by $s_{\alpha, n}$ is

$$
\widehat{H}_{\alpha, n}:=\left\{(v, \mu) \in \widehat{V}^{*} \mid\langle\alpha, v\rangle=\mu n\right\} .
$$


Set $\delta:=(0,1) \in \widehat{V}=V \oplus \mathbb{Q}$. Then

$$
\alpha_{n}:=(\alpha, 0)-n \delta \in \widehat{V}
$$

is an equation of $\widehat{H}_{\alpha, n}$. We call $\alpha_{n}$ a (real) affine root, and we set

$$
\widehat{R}:=\left\{\alpha_{n} \mid \alpha \in R, n \in \mathbb{Z}\right\}=R \times \mathbb{Z} \delta \subset \widehat{V} .
$$

Let us choose a system $R^{+} \subset R$ of positive (finite) roots. The corresponding set of positive affine roots is

$$
\widehat{R}^{+}:=\{\alpha+n \delta \mid \alpha \in R, n>0\} \cup\left\{\alpha \mid \alpha \in R^{+}\right\} .
$$

Then $\widehat{R}=\widehat{R}^{+} \dot{\cup}-\widehat{R}^{+}$and for any reflection $t \in \widehat{\mathcal{T}}$ there is a unique positive affine root $\alpha_{t}=\alpha-n \delta$ such that $t=s_{\alpha, n}$.

Now consider the dual action of $\widehat{\mathcal{W}}$ on $\widehat{V}$ that is given by $w \cdot \phi=\phi \circ w^{-1}$ for $w \in \widehat{\mathcal{W}}$ and $\phi \in \widehat{V}$. More explicitly, it is given by

$$
\begin{aligned}
w(0, \nu) & =(0, \nu), \\
s_{\alpha, n}(\lambda, 0) & =\left(s_{\alpha}(\lambda), n\left\langle\lambda, \alpha^{\vee}\right\rangle\right) .
\end{aligned}
$$

Denote by $\bar{\imath} \widehat{V} \rightarrow V$ the map $(\lambda, \nu) \mapsto \lambda$. Then the following is immediate.

Lemma 2.1. For each $w \in \widehat{\mathcal{W}}$ and $x \in \widehat{V}$ we have $\overline{w(x)}=\bar{w}(\bar{x})$.

Recall that the set of positive roots $R^{+}$determines a set of simple roots $\Delta \subset R^{+}$ and a set of simple reflections $\mathcal{S} \subset \mathcal{W}$. The corresponding set of simple affine reflections is

$$
\widehat{\mathcal{S}}:=\mathcal{S} \cup\left\{s_{\gamma, 1}\right\} \subset \widehat{\mathcal{W}}
$$

where $\gamma \in R^{+}$is the highest root. Then $(\widehat{\mathcal{W}}, \widehat{\mathcal{S}})$ is a Coxeter system and we denote by $l: \widehat{\mathcal{W}} \rightarrow \mathbb{N}$ the associated length function.

2.3. The associated moment graph. Let $Y \cong \mathbb{Z}^{r}$ be a lattice. An (unordered) moment graph $\mathcal{G}$ over $Y$ is given by a graph $(\mathcal{V}, \mathcal{E})$ with vertices $\mathcal{V}$ and edges $\mathcal{E}$ and a map $\alpha: \mathcal{E} \rightarrow Y \backslash\{0\}$ which is called the labeling.

Let $X:=\left\{\lambda \in V \mid\left\langle\lambda, \alpha^{\vee}\right\rangle \in \mathbb{Z} \quad \forall \alpha \in R\right\}$ be the weight lattice and $\widehat{X}:=$ $X \oplus \mathbb{Z} \delta \subset \widehat{V}$ the affine weight lattice. The latter contains the affine root lattice $\mathbb{Z} \widehat{R}$. The affine Bruhat graph $\widehat{\mathcal{G}}=\widehat{\mathcal{G}}_{R}$ associated to $R$ is given as follows. Its underlying lattice is $\widehat{X}$. The set of vertices is the affine Weyl group $\widehat{\mathcal{W}}$ and $x, y \in \widehat{\mathcal{W}}$ are connected by an edge if there is a reflection $t \in \widehat{\mathcal{T}}$ with $t x=y$. This edge is labelled by the positive affine root $\alpha_{t} \in \widehat{X}$ corresponding to $t$.

For a parabolic subgroup $\widehat{\mathcal{W}}_{I} \subset \widehat{\mathcal{W}}$ that is given by a subset $I$ of $\widehat{\mathcal{S}}$ we obtain another graph $\widehat{\mathcal{G}}^{I}$ as follows. Its set of vertices is $\widehat{\mathcal{W}}^{I}:=\widehat{\mathcal{W}} / \widehat{\mathcal{W}}_{I}$ and $x, y \in \widehat{\mathcal{W}}^{I}$ are connected by an edge if there exists $t \in \widehat{\mathcal{T}}$ such that $t x=y$. This edge is then labelled by $\alpha_{t}$.

2.4. The structure algebra. We denote by $\mathbb{Z}^{\prime}$ the subring $\mathbb{Z}\left[2^{-1}\right]$ of $\mathbb{Q}$. Let $S=S_{\mathbb{Z}^{\prime}}=S\left(X \otimes_{\mathbb{Z}} \mathbb{Z}^{\prime}\right)$ and $\widehat{S}=\widehat{S}_{\mathbb{Z}^{\prime}}=S\left(\widehat{X} \otimes_{\mathbb{Z}} \mathbb{Z}^{\prime}\right)$ be the symmetric algebras over the free $\mathbb{Z}^{\prime}$-modules associated to the lattices $X$ and $\widehat{X}$. Both $S$ and $\widehat{S}$ are $\mathbb{Z}$-graded algebras. The grading we choose is determined by setting $X \otimes_{\mathbb{Z}} \mathbb{Z}^{\prime}$ and $\widehat{X} \otimes_{\mathbb{Z}} \mathbb{Z}^{\prime}$ in degree 2 (this will become important only when we consider graded multiplicities). In this article many objects are considered to be $\mathbb{Z}$-graded. For 
$n \in \mathbb{Z}$ we denote by $(\cdot)\langle n\rangle$ the functor that shifts gradings by $n$; i.e., for a $\mathbb{Z}$-graded module $M=\bigoplus_{i \in \mathbb{Z}} M_{i}$ we have $M\langle n\rangle_{i}=M_{i+n}$.

The affine structure algebra associated to the root system $R$ is

$$
\widehat{\mathcal{Z}}=\widehat{\mathcal{Z}}_{\mathbb{Z}^{\prime}}:=\left\{\left(z_{w}\right) \in \prod_{w \in \widehat{\mathcal{W}}} \widehat{S} \mid \begin{array}{c}
z_{w} \equiv z_{s_{\alpha, n} w} \quad \bmod \alpha_{n} \\
\text { for all } \alpha \in R^{+}, n \in \mathbb{Z}, w \in \widehat{\mathcal{W}}
\end{array}\right\} .
$$

The infinite product $\prod_{w \in \widehat{\mathcal{W}}} \widehat{S}$ appearing in the definition above should be understood as the product in the category of graded $\widehat{S}$-modules; i.e., the product is taken degree-wise. As the defining relations are homogeneous, $\widehat{\mathcal{Z}}$ is a $\mathbb{Z}$-graded algebra. It is an $\widehat{S}$-algebra via the diagonal inclusion $\widehat{S} \subset \widehat{\mathcal{Z}}$.

Let $k$ be a ring in which 2 is invertible. For any $\mathbb{Z}^{\prime}$-module $M$ we set $M_{k}:=$ $M \otimes_{\mathbb{Z}^{\prime}} k$. In particular, we have $S_{k}=S\left(X \otimes_{\mathbb{Z}} k\right)$ and $\widehat{S}_{k}=S\left(\widehat{X} \otimes_{\mathbb{Z}} k\right)$, and the structure algebra over $k$ is

$$
\widehat{\mathcal{Z}}_{k}=\widehat{\mathcal{Z}} \otimes_{\mathbb{Z}^{\prime}} k .
$$

Note that this does not necessarily coincide with the subalgebra of $\prod_{w \in \widehat{\mathcal{W}}} \widehat{S}_{k}$ defined in analogy with $\widehat{\mathcal{Z}}$ by congruence relations.

Let us construct some elements in $\widehat{\mathcal{Z}}$. For $\lambda \in \widehat{X}$ and $x \in \widehat{\mathcal{W}}$ define $c(\lambda)_{x}:=$ $x(\lambda)$. As $s_{\alpha, n}(\mu) \equiv \mu \bmod \alpha_{n}$ for all $\alpha, n, \mu$, we have that $c(\lambda):=\left(c(\lambda)_{x}\right)_{x \in \widehat{\mathcal{W}}}$ is contained in $\widehat{\mathcal{Z}}$. This yields a homomorphism

$$
c: \widehat{X} \rightarrow \widehat{\mathcal{Z}}
$$

of abelian groups. Note that the image of $c$ is contained in degree 2 .

For $\beta \in R^{+}$let $\widehat{\mathcal{W}}^{\beta} \subset \widehat{\mathcal{W}}$ be the subgroup generated by all reflections $s_{\beta, n}$ with $n \in \mathbb{Z}$. The next lemma will be used in the proof of Theorem 5.4.

Lemma 2.2. For each $\beta \in R^{+}$and $w \in \widehat{\mathcal{W}}$ there is an element $z=\left(z_{x}\right)_{x \in \widehat{\mathcal{W}}} \in \widehat{\mathcal{Z}}$ of degree 2 with the following properties:

(1) $z_{w}=0$,

(2) for all $x \in \widehat{\mathcal{W}}^{\beta}$ we have $z_{x w} \in \mathbb{Z}^{\prime} \delta$ if $l(x)$ is even, and $z_{x w} \in \beta+\mathbb{Z}^{\prime} \delta$ if $l(x)$ is odd.

Proof. For all $x \in \widehat{\mathcal{W}}$ set $\tilde{z}_{x}:=\beta-x w^{-1}(\beta) \in \widehat{X} \subset \widehat{S}$. Then $\tilde{z}:=\left(\tilde{z}_{x}\right)_{x \in \widehat{\mathcal{W}}}=$ $\beta \cdot 1_{\widehat{\mathcal{Z}}}+c\left(w^{-1}(\beta)\right)$ is an element in $\widehat{\mathcal{Z}}$. Obviously, $\tilde{z}_{w}=0$. For all $x \in \widehat{\mathcal{W}}^{\beta}$ we calculate (using Lemma 2.1):

$$
\begin{aligned}
\tilde{z}_{x w} & =\beta-x(\beta) \\
& \equiv \beta-\bar{x}(\beta) \bmod \delta \\
& \equiv \beta-(-1)^{l(x)} \beta \bmod \delta,
\end{aligned}
$$

and hence $2^{-1} \tilde{z}$ serves our purpose.

2.5. Invariants. Choose $t \in \widehat{\mathcal{T}}$ and consider the involution $w \mapsto w t$ on the set $\widehat{\mathcal{W}}$. We denote by $\sigma_{t}$ the algebra involution on $\prod_{w \in \widehat{\mathcal{W}}} \widehat{S}$ that is given by switching coordinates: $\sigma_{t}\left(z_{w}\right)=\left(z_{w}^{\prime}\right)$, where $z_{w}^{\prime}=z_{w t}$. Then the subalgebra $\widehat{\mathcal{Z}} \subset \prod_{w \in \widehat{\mathcal{W}}} \widehat{S}$ is $\sigma_{t}$-stable. Denote by $\widehat{\mathcal{Z}}^{t} \subset \widehat{\mathcal{Z}}$ the subalgebra of $\sigma_{t}$-invariants. For the ring $k$ we set $\widehat{\mathcal{Z}}_{k}^{t}=\left(\widehat{\mathcal{Z}}^{t}\right)_{k}$. We denote by $\widehat{\mathcal{Z}}^{-t} \subset \widehat{\mathcal{Z}}$ the $\widehat{\mathcal{Z}}^{t}$-module of $\sigma_{t}$-anti-invariants. As we can divide by 2 in $\widehat{\mathcal{Z}}$ we have $\widehat{\mathcal{Z}}=\widehat{\mathcal{Z}}^{t} \oplus \widehat{\mathcal{Z}}^{-t}$. Consider the element $c\left(\alpha_{t}\right) \in \widehat{\mathcal{Z}}$. Then $\sigma_{t}\left(c\left(\alpha_{t}\right)\right)=-c\left(\alpha_{t}\right)$ as $t\left(\alpha_{t}\right)=-\alpha_{t}$. So $c\left(\alpha_{t}\right) \in \widehat{\mathcal{Z}}^{-t}$. 
Lemma 2.3. We have $\widehat{\mathcal{Z}}=\widehat{\mathcal{Z}}^{t} \oplus c\left(\alpha_{t}\right) \widehat{\mathcal{Z}}^{t}$ and $\widehat{\mathcal{Z}}_{k}=\widehat{\mathcal{Z}}_{k}^{t} \oplus c\left(\alpha_{t}\right) \widehat{\mathcal{Z}}_{k}^{t}$.

Proof. The second claim is an immediate consequence of the first. We clearly have $c\left(\alpha_{t}\right) \widehat{\mathcal{Z}}^{t} \subset \widehat{\mathcal{Z}}^{-t}$. We now show that this inclusion is a bijection. Let $z \in \widehat{\mathcal{Z}}^{-t}$. Then $z_{w}=-z_{w t}$ and $z_{w} \equiv z_{w t} \bmod \alpha_{w t w^{-1}}$; hence $z_{w}$ is divisible by $\alpha_{w t w^{-1}}=$ $\pm w\left(\alpha_{t}\right)= \pm c\left(\alpha_{t}\right)_{w}$ in $\widehat{S}$. Hence $z^{\prime}=c\left(\alpha_{t}\right)^{-1} z$ is a well-defined element in $\prod_{w \in \widehat{\mathcal{W}}} \widehat{S}$. Moreover, $z^{\prime}$ is $\sigma_{t}$-invariant as $c\left(\alpha_{t}\right)$ and $z$ are anti-invariant. We have to show that $z^{\prime} \in \widehat{\mathcal{Z}}$

Let $w \in \widehat{\mathcal{W}}$ and $u \in \widehat{\mathcal{T}}$. Then

$$
\begin{aligned}
c\left(\alpha_{t}\right)_{u w} c\left(\alpha_{t}\right)_{w}\left(z_{u w}^{\prime}-z_{w}^{\prime}\right) & =c\left(\alpha_{t}\right)_{w} z_{u w}-c\left(\alpha_{t}\right)_{u w} z_{w} \\
& =\left(c\left(\alpha_{t}\right)_{w}-c\left(\alpha_{t}\right)_{u w}\right) z_{u w}+c\left(\alpha_{t}\right)_{u w}\left(z_{u w}-z_{w}\right)
\end{aligned}
$$

is divisible by $\alpha_{u}$, since the expressions in brackets on the right-hand side are. If $u \neq w t w^{-1}$, then neither $c\left(\alpha_{t}\right)_{u w}$ nor $c\left(\alpha_{t}\right)_{w}$ is divisible by $\alpha_{u}$, so we deduce $z_{u w}^{\prime} \equiv z_{w}^{\prime} \bmod \alpha_{u}$. If $u=w t w^{-1}$, then $z_{u w}^{\prime}=z_{w}^{\prime}$ as $z^{\prime}$ is $\sigma_{t}$-invariant. So $z_{u w}^{\prime} \equiv z_{w}^{\prime}$ $\bmod \alpha_{u}$ in any case; hence $z^{\prime} \in \widehat{\mathcal{Z}}$.

2.6. Translation functors and special modules. Let $\widehat{\mathcal{Z}}_{k}$-mod ${ }^{f}$ be the category of $\mathbb{Z}$-graded $\widehat{\mathcal{Z}}_{k}$-modules that are torsion-free over $\widehat{S}_{k}$ and finitely generated over $\widehat{S}_{k}$. For a simple affine reflection $s \in \widehat{\mathcal{S}}$ define $\widehat{\mathcal{Z}}_{k}^{s}$-mod ${ }^{f}$ analogously. The restriction functor $\vartheta_{o n}^{s}:=\operatorname{Res} \widehat{\mathcal{Z}}_{\widehat{\mathcal{Z}}_{k}}^{\widehat{x}_{k}}$ is a functor from $\widehat{\mathcal{Z}}_{k}$-mod ${ }^{f}$ to $\widehat{\mathcal{Z}}_{k}^{s}$-mod ${ }^{f}$. By the previous lemma, the induction functor $\vartheta_{\text {out }}^{s}:=\operatorname{Ind}_{\widehat{\mathcal{Z}}_{k}^{s}}^{\widehat{\mathcal{Z}}_{k}}=\widehat{\mathcal{Z}}_{k} \otimes_{\widehat{\mathcal{Z}}_{k}^{s}}$. is a functor from $\widehat{\mathcal{Z}}_{k}^{s}$-mod ${ }^{f}$ to $\widehat{\mathcal{Z}}_{k}$-mod ${ }^{f}$. We denote by $\theta^{s}:=\vartheta_{\text {out }}^{s} \circ \vartheta_{\text {on }}^{s}: \widehat{\mathcal{Z}}_{k}$-mod ${ }^{f} \rightarrow \widehat{\mathcal{Z}}_{k}$-mod ${ }^{f}$ the composition. Let $B_{e} \in \widehat{\mathcal{Z}}_{k}$-mod ${ }^{f}$ be the free $\widehat{S}_{k}$-module of rank one on which $z=\left(z_{w}\right)$ acts by multiplication with $z_{e}$.

Definition 2.4. (1) The category of special $\widehat{\mathcal{Z}}_{k}$-modules is the full subcategory $\mathcal{H}_{k}$ of $\widehat{\mathcal{Z}}_{k}$-mod ${ }^{f}$ that consists of all objects that are isomorphic to a direct summand of a direct sum of modules of the form $\theta^{s} \circ \cdots \circ \theta^{t}\left(B_{e}\right)\langle n\rangle$, where $s, \ldots, t$ is an arbitrary sequence in $\widehat{\mathcal{S}}$ and $n$ is in $\mathbb{Z}$.

(2) Let $s \in \widehat{\mathcal{S}}$. The category of special $\widehat{\mathcal{Z}}_{k}^{s}$-modules is the full subcategory $\mathcal{H}_{k}^{s}$ of $\widehat{\mathcal{Z}}_{k}^{s}$-mod ${ }^{f}$ that consists of all objects that are isomorphic to a direct summand of $\vartheta_{o n}^{s}(M)$ for some $M \in \mathcal{H}_{k}$.

By definition, we have induced functors $\vartheta_{\text {on }}^{s}: \mathcal{H}_{k} \rightarrow \mathcal{H}_{k}^{s}, \vartheta_{\text {out }}^{s}: \mathcal{H}_{k}^{s} \rightarrow \mathcal{H}_{k}$ and $\theta^{s}: \mathcal{H}_{k} \rightarrow \mathcal{H}_{k}$ for all $s \in \widehat{\mathcal{S}}$. For $M \in \mathcal{H}_{\mathbb{Z}^{\prime}}$ and $N \in \mathcal{H}_{\mathbb{Z}^{\prime}}^{s}$ we have $\left(\vartheta_{o n}^{s} M\right)_{k}=$ $\vartheta_{o n}^{s}\left(M_{k}\right)$ and $\left(\vartheta_{\text {out }}^{s} M\right)_{k}=\vartheta_{\text {out }}^{s}\left(M_{k}\right)$; hence base change yields functors from $\mathcal{H}_{\mathbb{Z}^{\prime}}$ to $\mathcal{H}_{k}$ and from $\mathcal{H}_{\mathbb{Z}^{\prime}}^{s}$ to $\mathcal{H}_{k}^{s}$.

For the applications we need the ring $k$ to be a field, preferably of positive characteristic. But for some of the arguments in the following we would need the GKM-restriction on $k$ (cf. Section 9.1). As we prefer to consider, for the moment, the whole graph $\widehat{\mathcal{G}}$, this restricts ourselves to the characteristic zero case. A good compromise is to work over the ring $\mathbb{Z}^{\prime}$. So in the following, when we leave out the index $k$, we mean objects over $\mathbb{Z}^{\prime}$. Later (cf. Section 4.4) we use the base change functor to establish the crucial results for fields $k$.

2.7. Finiteness of special modules. The next lemma shows that we can consider each special module as a module over a finite version of the structure algebra. This will be important for the localization constructions in the next section. 
For $\Omega \subset \widehat{\mathcal{W}}$ define

$$
\widehat{\mathcal{Z}}(\Omega):=\left\{\left(z_{w}\right) \in \prod_{w \in \Omega} \widehat{S} \mid \begin{array}{c}
z_{w} \equiv z_{t w} \bmod \alpha_{t} \\
\text { for all } t \in \widehat{\mathcal{T}}, w \in \Omega \text { with } t w \in \Omega
\end{array}\right\} .
$$

The projection $\prod_{w \in \widehat{\mathcal{W}}} \widehat{S} \rightarrow \prod_{w \in \Omega} \widehat{S}$ with kernel $\prod_{w \in \widehat{\mathcal{W}} \backslash \Omega} \widehat{S}$ induces a map $\widehat{\mathcal{Z}} \rightarrow$ $\widehat{\mathcal{Z}}(\Omega)$. Note that this map need not be surjective. (If it were, this article, as well as many others, would be rather pointless.)

Choose $s \in \widehat{\mathcal{S}}$. In the following we call a subset $\Omega$ of $\widehat{\mathcal{W}} s$-invariant if $x \in \Omega$ implies $x s \in \Omega$. For an $s$-invariant $\Omega$ we can define, as before, an algebra involution $\sigma_{s}$ on $\widehat{\mathcal{Z}}(\Omega)$. Then we let $\widehat{\mathcal{Z}}(\Omega)^{s} \subset \widehat{\mathcal{Z}}(\Omega)$ be the subalgebra of invariants. There is a canonical map $\widehat{\mathcal{Z}}^{s} \rightarrow \widehat{\mathcal{Z}}(\Omega)^{s}$. The proof of Lemma 2.3 carries over and yields a decomposition $\widehat{\mathcal{Z}}(\Omega)=\widehat{\mathcal{Z}}(\Omega)^{s} \oplus c\left(\alpha_{s}\right) \widehat{\mathcal{Z}}(\Omega)^{s}$.

Lemma 2.5. (1) Let $M$ be an object in $\mathcal{H}$. Then there exists a finite subset $\Omega$ of $\widehat{\mathcal{W}}$ and an action of $\widehat{\mathcal{Z}}(\Omega)$ on $M$ such that $\widehat{\mathcal{Z}}$ acts on $M$ via the canonical map $\widehat{\mathcal{Z}} \rightarrow \widehat{\mathcal{Z}}(\Omega)$.

(2) Let $s \in \widehat{\mathcal{S}}$ and let $N$ be an object in $\mathcal{H}^{s}$. Then there exists a finite, $s$ invariant subset $\Omega$ of $\widehat{\mathcal{W}}$ and an action of $\widehat{\mathcal{Z}}(\Omega)^{s}$ on $N$ such that $\widehat{\mathcal{Z}}^{s}$ acts on $N$ via the canonical map $\widehat{\mathcal{Z}}^{s} \rightarrow \widehat{\mathcal{Z}}(\Omega)^{s}$.

Proof. Suppose we have proven (1). Then (2) follows, since it is enough to prove (2) for modules of the form $\vartheta_{\text {on }}^{s}(M)$ with $M \in \mathcal{H}$, and if $M$ is acted upon by $\widehat{\mathcal{Z}}(\Omega)$, then $\vartheta_{\text {on }}^{s}(M)$ is acted upon by $\widehat{\mathcal{Z}}(\Omega \cup \Omega s)^{s}$.

Now (1) certainly holds for $M=B_{e}$ with $\Omega=\{e\}$. It is enough to show that if (1) holds for $M$, then it also holds for $\theta^{s}(M)$ for all $s \in \widehat{\mathcal{S}}$. So assume (1) holds for $M$ and choose $s \in \widehat{\mathcal{S}}$. Choose $\Omega \subset \widehat{\mathcal{W}}$ with the desired property for $M$. After enlarging $\Omega$ if necessary, we can assume that $\Omega$ is $s$-invariant. Then the action of $\widehat{\mathcal{Z}}^{s}$ on $\vartheta_{\text {on }}^{s} M$ factors over an action of $\widehat{\mathcal{Z}}(\Omega)^{s}$, and since $\theta^{s}(M)=\widehat{\mathcal{Z}} \otimes_{\widehat{\mathcal{Z}}^{s}} M=\widehat{\mathcal{Z}}(\Omega) \otimes_{\widehat{\mathcal{Z}}(\Omega)^{s}} M$ we get an action of $\widehat{\mathcal{Z}}(\Omega)$ on $\theta^{s}(M)$ with the claimed property.

2.8. Sheaves on moment graphs. We need another, more local, construction of the special $\widehat{\mathcal{Z}}$-modules as global sections of certain sheaves on the moment graph associated to our data. Let us recall the notion of a sheaf on such a graph. So let $\mathcal{G}$ be a moment graph over the lattice $Y$. Let $k$ be a ring and denote by $S_{k}(Y):=$ $S\left(Y \otimes_{\mathbb{Z}} k\right)$ the symmetric algebra over the free $k$-module associated to $Y$, again graded in such a way that $\operatorname{deg} Y \otimes_{\mathbb{Z}} k=2$. A $k$-sheaf $\mathscr{F}=\left(\left\{\mathscr{F}^{x}\right\},\left\{\mathscr{F}^{E}\right\},\left\{\rho_{x, E}\right\}\right)$ on $\mathcal{G}$ is given by the following data:

- an $S_{k}(Y)$-module $\mathscr{F}^{x}$ for any vertex $x$,

- an $S_{k}(Y)$-module $\mathscr{F}^{E}$ for any edge $E$ with the property $\alpha(E) \cdot \mathscr{F}^{E}=0$,

- an $S_{k}(Y)$-module homomorphism $\rho_{x, E}: \mathscr{F}^{x} \rightarrow \mathscr{F}^{E}$ for any vertex $x$ adjacent to the edge $E$.

A homomorphism $f: \mathscr{F} \rightarrow \mathscr{G}$ between $k$-sheaves on $\mathcal{G}$ is given by $S_{k}(Y)$-module homomorphisms $f^{x}: \mathscr{F}^{x} \rightarrow \mathscr{G}^{x}$ for any vertex $x$ and $f^{E}: \mathscr{F}^{E} \rightarrow \mathscr{G}^{E}$ for any edge $E$ that are compatible with all $\rho$-maps. We denote by $\mathcal{G}$ - $\bmod _{k}$ the resulting category of $k$-sheaves on $\mathcal{G}$. Recall that we assume that each $S_{k}(Y)$-module and each homomorphism is graded. This yields a graded structure on $\mathcal{G}$ - $\bmod _{k}$ with the obvious shift functor. 
Let $\mathscr{F}$ be a $k$-sheaf on $\mathcal{G}$ and let $\Omega$ be a subset of the set $\mathcal{V}$ of vertices of $\mathcal{G}$. The sections of $\mathscr{F}$ over $\Omega$ are defined as

$$
\Gamma(\Omega, \mathscr{F}):=\left\{\begin{array}{l|l}
\left(m_{x}\right) \in \prod_{x \in \Omega} \mathscr{F}^{x} & \begin{array}{c}
\rho_{x, E}\left(m_{x}\right)=\rho_{y, E}\left(m_{y}\right) \\
\text { for any } x, y \in \Omega \text { that are } \\
\text { connected by the edge } E
\end{array}
\end{array}\right\} .
$$

We denote by $\Gamma(\mathscr{F}):=\Gamma(\mathcal{V}, \mathscr{F})$ the space of global sections of $\mathscr{F}$.

2.9. Translation functors on sheaves. In the following we construct an analog of the functors $\vartheta_{\text {on }}^{s}$ and $\vartheta_{\text {out }}^{s}$ for the categories of sheaves on the moment graphs $\widehat{\mathcal{G}}$ and $\widehat{\mathcal{G}}^{s}$ associated to the root system $R$ and a simple affine reflection $s$. Again we restrict ourselves to the case $k=\mathbb{Z}^{\prime}$ and leave out the index $k$. The word sheaf means $\mathbb{Z}^{\prime}$-sheaf.

So let $\mathscr{F}$ be a sheaf on $\widehat{\mathcal{G}}$. We define the sheaf $\mathscr{G}=\widetilde{\vartheta}_{o n}^{s} \mathscr{F}$ on $\widehat{\mathcal{G}}^{s}$ as follows. For $\bar{x}=\{x, x s\} \in \widehat{\mathcal{W}}^{s}=\widehat{\mathcal{W}} /\{1, s\}$ set $\mathscr{G}^{\bar{x}}:=\Gamma(\{x, x s\}, \mathscr{F})$. Let $\bar{E}$ be an edge connecting $\bar{x}=\{x, x s\}$ and $\bar{y}=\{y, y s\}$. Then there is $t \in \widehat{\mathcal{T}}$ with $t \bar{x}=\bar{y}$. We can assume that $y=t x$, so there is an edge $E$ connecting $y$ and $x$ and another edge $E s$ connecting ys and $x s$, both labelled by $\alpha_{t}$. We set $\mathscr{G}^{E}:=\mathscr{F}^{E} \oplus \mathscr{F}^{E s}$. For a vertex $\bar{x}$ adjacent to $\bar{E}$ we let $\rho_{\bar{x}, \bar{E}}$ be the composition of the inclusion $\Gamma(\{x, x s\}, \mathscr{F}) \subset$ $\mathscr{F}^{x} \oplus \mathscr{F}^{x s}$ with the direct sum $\rho_{x, E} \oplus \rho_{x s, E s}: \mathscr{F}^{x} \oplus \mathscr{F}^{x s} \rightarrow \mathscr{F}^{E} \oplus \mathscr{F}^{E s}$. These data define a sheaf $\mathscr{G}$ on $\widehat{\mathcal{G}}^{s}$. One immediately checks that this yields a functor $\widetilde{\vartheta}_{\text {on }}^{s}: \widehat{\mathcal{G}}$-mod $\rightarrow \widehat{\mathcal{G}}^{s}$-mod.

Now let $\mathscr{G}$ be a sheaf on $\widehat{\mathcal{G}}^{s}$. We define a sheaf $\mathscr{F}=\widetilde{\vartheta}_{\text {out }}^{s} \mathscr{G}$ on $\widehat{\mathcal{G}}$. For a vertex $x$ on $\widehat{\mathcal{G}}$ let $\bar{x}=\{x, x s\}$ be the corresponding vertex on $\widehat{\mathcal{G}}^{s}$. Then we set $\mathscr{F}^{x}:=\mathscr{G}^{\bar{x}}$. Let $E$ be an edge of $\widehat{\mathcal{G}}$ connecting $x$ and $y$. In the case $y=x s$, so $\bar{x}=\bar{y}$, we set $\mathscr{F}^{E}:=\mathscr{G}^{\bar{x}} / \alpha(E) \mathscr{G}^{\bar{x}}$ and we let $\rho_{x, E}$ and $\rho_{y, E}$ be the canonical maps $\mathscr{G}^{\bar{x}} \rightarrow \mathscr{G}^{\bar{x}} / \alpha(E) \mathscr{G}^{\bar{x}}$. If $y \neq x s$, then $\bar{x}$ and $\bar{y}$ are connected by an edge $\bar{E}$ and we set $\mathscr{F}^{E}:=\mathscr{G}^{\bar{E}}$ and $\rho_{x, E}:=\rho_{\bar{x}, \bar{E}}, \rho_{y, E}:=\rho_{\bar{y}, \bar{E}}$. This defines a sheaf $\mathscr{F}$ on $\widehat{\mathcal{G}}$ and it is immediate that we obtain a functor $\widetilde{\vartheta}_{\text {out }}^{s}: \widehat{\mathcal{G}}^{s}$-mod $\rightarrow \widehat{\mathcal{G}}$-mod. We denote by $\tilde{\theta}^{s}=\widetilde{\vartheta}_{\text {out }}^{s} \circ \widetilde{\vartheta}_{\text {on }}^{s}: \widehat{\mathcal{G}}$-mod $\rightarrow \widehat{\mathcal{G}}$-mod the composition.

Let $\Omega$ be a subset of $\widehat{\mathcal{W}}$. Note that coordinatewise multiplication yields a $\widehat{\mathcal{Z}}(\Omega)$ module structure on $\Gamma(\Omega, \mathscr{F})$ for any sheaf $\mathscr{F}$ on $\widehat{\mathcal{G}}$. Likewise, if $\Omega$ is $s$-invariant and if $\bar{\Omega} \in \widehat{\mathcal{W}}^{s}$ is its image, then $\Gamma(\bar{\Omega}, \mathscr{G})$ is a $\widehat{\mathcal{Z}}(\Omega)^{s}$-module for each sheaf $\mathscr{G}$ on $\widehat{\mathcal{G}}^{s}$.

Lemma 2.6. Suppose that $\Omega \subset \widehat{\mathcal{W}}$ is s-invariant and denote by $\bar{\Omega} \in \widehat{\mathcal{W}}^{s}$ its canonical image. For any sheaf $\mathscr{F}$ on $\widehat{\mathcal{G}}$ we have $\Gamma\left(\bar{\Omega}, \widetilde{\vartheta}_{o n}^{s} \mathscr{F}\right)=\vartheta_{o n}^{s} \Gamma(\Omega, \mathscr{F})$. For any sheaf $\mathscr{G}$ on $\widehat{\mathcal{G}}^{s}$ such that $\mathscr{G}^{x}$ is a free $\widehat{S}$-module for any vertex and for any edge $\bar{E}$ the stalk $\mathscr{G}^{\bar{E}}$ is a free $\widehat{S} / \alpha(E) \widehat{S}$-module, we have $\Gamma\left(\Omega, \widetilde{\vartheta}_{\text {out }}^{s} \mathscr{G}\right)=\vartheta_{\text {out }}^{\text {s }} \Gamma(\bar{\Omega}, \mathscr{G})$.

Proof. Note that from the definition of $\widetilde{\vartheta}_{o n}^{s}$ it immediately follows that we have an identification $\Gamma(\Omega, \mathscr{F})=\Gamma\left(\bar{\Omega}, \widetilde{\vartheta}_{\text {on }}^{s} \mathscr{F}\right)$ as $\widehat{\mathcal{Z}}(\Omega)^{s}$-modules, which yields the first claim.

In order to prove the second claim, let us set $\mathscr{F}=\widetilde{\vartheta}_{\text {out }}^{s} \mathscr{G}$. We then have $\mathscr{F}^{x}=$ $\mathscr{F}^{x s}$ for any $x \in \Omega$. Hence we can define an involution $\sigma_{s}$ on $\prod_{x \in \Omega} \mathscr{F}^{x}$ by $\sigma_{s}\left(m_{x}\right)=$ $\left(m_{x}^{\prime}\right)$, where $m_{x}^{\prime}=m_{x s}$ for any $x$. It follows from the definitions that $\Gamma(\Omega, \mathscr{F}) \subset$ $\prod_{x \in \Omega} \mathscr{F}^{x}$ is $\sigma_{s^{-}}$-stable. Hence, we have a decomposition $\Gamma(\Omega, \mathscr{F})=\Gamma(\Omega, \mathscr{F})^{s} \oplus$ $\Gamma(\Omega, \mathscr{F})^{-s}$ into $\sigma_{s}$-invariants and $\sigma_{s^{-}}$anti-invariants. 
We now prove that $\Gamma(\Omega, \mathscr{F})^{-s}=c\left(\alpha_{s}\right) \Gamma(\Omega, \mathscr{F})^{s}$. The arguments are similar to the ones used for Lemma 2.3. Let $x \in \Omega$ and let $E$ be the edge connecting $x$ and $x s$. We have $\mathscr{F}^{x}=\mathscr{F}^{x s}=\mathscr{G}^{\bar{x}}$ and $\mathscr{F}^{E}=\mathscr{G}^{\bar{x}} / \alpha(E) \mathscr{G}^{\bar{x}}$. If $m=\left(m_{x}\right) \in \Gamma(\Omega, \mathscr{F})^{-s}$, then $m_{x}=-m_{x s}$ and $m_{x} \equiv m_{x s} \bmod \alpha(E)$. Hence $m_{x}$ and $m_{x s}$ are divisible by $\alpha(E)$ in $\mathscr{G}^{\bar{x}}$. Now $\alpha(E)$ is the positive root corresponding to the reflection $x s x^{-1}$; i.e., $\alpha(E)= \pm x\left(\alpha_{s}\right)= \pm c\left(\alpha_{s}\right)_{x}$. So $m^{\prime}=c\left(\alpha_{s}\right)^{-1} m$ is a well-defined element in $\prod_{x \in \Omega} \mathscr{F}^{x}$, which is, moreover, $\sigma_{s}$-invariant. Hence it remains to show that $m^{\prime}$ is a section in $\Gamma(\Omega, \mathscr{F})$.

So let $E$ now be an edge connecting $x$ and $y$. We want to show that $\rho_{x, E}\left(m_{x}^{\prime}\right)=$ $\rho_{y, E}\left(m_{y}^{\prime}\right)$. In the case $y=x s$ this is clear. So suppose that $y \neq x s$. As $m$ is a section, we have $\rho_{x, E}\left(m_{x}\right)=\rho_{y, E}\left(m_{y}\right)$. By our assumption, $\mathscr{F}^{E}=\mathscr{G}^{\bar{E}}$ is a free $\widehat{S} / \alpha(E) \widehat{S}$-module. Now $c\left(\alpha_{s}\right)_{x}$ and $c\left(\alpha_{s}\right)_{y}$ are equivalent modulo $\alpha(E)$; hence they yield the same element in $\widehat{S} / \alpha(E) \widehat{S}$, which is not a zero divisor (here the GKMrestriction is needed in case we worked over a field of positive characteristic). We deduce that $\rho_{x, E}\left(m_{x}^{\prime}\right)=\rho_{y, E}\left(m_{y}^{\prime}\right)$. Hence $m^{\prime}$ is a section of $\mathscr{F}$. So we showed that $\Gamma(\Omega, \mathscr{F})^{-s}=c\left(\alpha_{s}\right) \Gamma(\Omega, \mathscr{F})^{s}$; hence we have $\Gamma(\Omega, \mathscr{F})=\Gamma(\Omega, \mathscr{F})^{s} \oplus c\left(\alpha_{s}\right) \Gamma(\Omega, \mathscr{F})^{s}$.

From the definition of $\widetilde{\vartheta}_{\text {out }}^{s}$ it is immediate that $\Gamma(\Omega, \mathscr{F})^{s}$ can be identified with $\Gamma(\bar{\Omega}, \mathscr{G})$ as a $\widehat{\mathcal{Z}}^{s}$-module. Using Lemma 2.3 we get an identification $\Gamma(\Omega, \mathscr{F})=$ $\Gamma(\bar{\Omega}, \mathscr{G}) \oplus c\left(\alpha_{s}\right) \Gamma(\bar{\Omega}, \mathscr{G})=\vartheta_{\text {out }}^{s} \Gamma(\bar{\Omega}, \mathscr{G})$ of $\widehat{\mathcal{Z}}$-modules.

2.10. Special sheaves on moment graphs. We let $\mathscr{B}_{e}$ be the following sheaf on $\widehat{\mathcal{G}}$. We set $\mathscr{B}_{e}^{e}=\widehat{S}$ and $\mathscr{B}_{e}^{x}=0$ for $x \neq e$. For any edge $E$ we set $\mathscr{B}_{e}^{E}=0$ and we let all $\rho_{x, E}$ 's be the zero homomorphism.

Definition 2.7. (1) The category of special sheaves on $\widehat{\mathcal{G}}$ is the full subcategory $\mathcal{A}$ of $\widehat{\mathcal{G}}-\bmod _{\mathbb{Z}^{\prime}}$ that consists of all objects that are isomorphic to a direct summand of a direct sum of sheaves of the form $\tilde{\theta}^{s} \circ \cdots \circ \tilde{\theta}^{t}\left(\mathscr{B}_{e}\right)\langle n\rangle$, where $s, \ldots, t$ is an arbitrary sequence in $\widehat{\mathcal{S}}$ and $n$ is in $\mathbb{Z}$.

(2) Let $s \in \widehat{\mathcal{S}}$. The category of special sheaves on $\widehat{\mathcal{G}}^{s}$ is the full subcategory $\mathcal{A}^{s}$ of $\widehat{\mathcal{G}}^{s}-\bmod _{\mathbb{Z}^{\prime}}$ that consists of all objects that are isomorphic to a direct summand of $\widetilde{\vartheta}_{\text {on }}^{s}(\mathscr{F})$ for some $\mathscr{F} \in \mathcal{A}$.

By definition, we have induced functors $\widetilde{\vartheta}_{\text {on }}^{s}: \mathcal{A} \rightarrow \mathcal{A}^{s}, \widetilde{\vartheta}_{\text {out }}^{s}: \mathcal{A}^{s} \rightarrow \mathcal{A}$ and $\tilde{\theta}^{s}: \mathcal{A} \rightarrow \mathcal{A}$ for all $s \in \widehat{\mathcal{S}}$. From the definitions of the translation functors it follows that for each $\mathscr{F} \in \mathcal{A}$ and each $\mathscr{G} \in \mathcal{A}^{s}$ the stalks $\mathscr{F}^{x}$ and $\mathscr{G}^{\bar{x}}$ are free $\widehat{S}$-modules of finite rank, and $\mathscr{F}^{E}$ and $\mathscr{G}^{E}$ are free $\widehat{S} / \alpha(E) \widehat{S}$-modules of finite rank.

2.11. The global sections of special sheaves. Note that we use a simplified definition of the translation functors. In general, the special sheaves that we obtain are not generated by global sections in the sense of [Fie08a, so are not BradenMacPherson sheaves. However, this definition nevertheless serves our purpose which is to interpret the special $\widehat{\mathcal{Z}}$-modules as global sections of moment graph sheaves. This is used in the proof of Lemma 4.2 in an essential way.

Proposition 2.8. (1) For $\mathscr{F} \in \mathcal{A}$ we have $\Gamma(\mathscr{F}) \in \mathcal{H}$. For any $s \in \widehat{\mathcal{S}}$ and $\mathscr{G} \in \mathcal{A}^{s}$ we have $\Gamma(\mathscr{G}) \in \mathcal{H}^{s}$.

(2) Let $s \in \widehat{\mathcal{S}}$. Then the following diagrams of functors commute: 

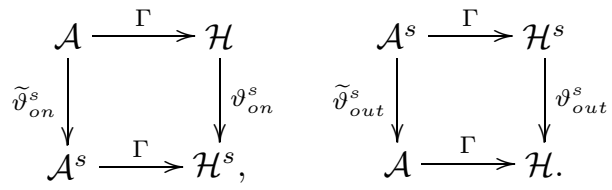

Proof. It follows immediately from the definitions that $\Gamma\left(\mathscr{B}_{e}\right) \cong B_{e}$ as $\widehat{\mathcal{Z}}$-modules. In order to prove our claims it is enough to show that for $\mathscr{F} \in \mathcal{A}$ with $\Gamma(\mathscr{F}) \in \mathcal{H}$ we have $\Gamma\left(\widetilde{\vartheta}_{o n}^{s} \mathscr{F}\right) \cong \vartheta_{\text {on }}^{s} \Gamma(\mathscr{F})$ and $\Gamma\left(\widetilde{\vartheta}_{\text {out }}^{s} \widetilde{\vartheta}_{\text {on }}^{s} \mathscr{F}\right) \cong \vartheta_{\text {out }}^{s} \Gamma\left(\widetilde{\vartheta}_{\text {on }}^{s} \mathscr{F}\right)$. For the first claim we can directly apply Lemma 2.6. As each stalk of a special sheaf is free (by construction), we can apply the same lemma to prove the second claim.

\section{Localization of SPECIAL MOdUles}

Each special $\widehat{\mathcal{Z}}$-module is naturally a module over the symmetric algebra $\widehat{S}$. In this section we collect some results on what happens if we invert some of the affine roots. In previous papers we used to work with certain localizations at prime ideals of the underlying symmetric algebra. But since the main aim of this paper is to relate the category of special modules to the category defined by Andersen, Jantzen and Soergel, it is convenient to use the following subalgebras of the quotient field of $\widehat{S}$. Let us fix $\beta \in R^{+}$and let us set

$$
\widehat{S}^{\emptyset}:=\widehat{S}\left[\alpha_{n}^{-1} \mid \alpha \in R^{+}, n \in \mathbb{Z}\right] \text { and } \widehat{S}^{\beta}:=\widehat{S}\left[\alpha_{n}^{-1} \mid \alpha \in R^{+}, \alpha \neq \beta, n \in \mathbb{Z}\right] .
$$

For a subset $\Omega$ of $\widehat{\mathcal{W}}$ set $\widehat{\mathcal{Z}}^{\emptyset}(\Omega):=\widehat{\mathcal{Z}}(\Omega) \otimes_{\widehat{S}} \widehat{S}^{\emptyset}$ and $\widehat{\mathcal{Z}}^{\beta}(\Omega):=\widehat{\mathcal{Z}}(\Omega) \otimes_{\widehat{S}} \widehat{S}^{\beta}$.

Recall that we defined the subgroup $\widehat{\mathcal{W}}^{\beta}$ of $\widehat{\mathcal{W}}$ that is generated by all $s_{\beta, n}$, $n \in \mathbb{Z}$. We denote by $\widehat{\mathcal{W}}^{\beta} \backslash \widehat{\mathcal{W}}$ the set of orbits in $\widehat{\mathcal{W}}$ of the left $\widehat{\mathcal{W}}^{\beta}$-action.

Lemma 3.1. For a finite subset $\Omega$ we have canonical isomorphisms

$$
\begin{aligned}
\widehat{\mathcal{Z}}^{\emptyset}(\Omega) & =\bigoplus_{w \in \Omega} \widehat{S}^{\emptyset}, \\
\widehat{\mathcal{Z}}^{\beta}(\Omega) & =\left\{\left(z_{w}\right) \in \bigoplus_{w \in \Omega} \widehat{S}^{\beta} \mid \begin{array}{c}
z_{w} \equiv z_{s_{\beta, n} w} \bmod \beta_{n} \\
\text { for all } w \in \Omega, n \in \mathbb{Z} \text { with } s_{\beta, n} w \in \Omega
\end{array}\right\} \\
& =\bigoplus_{\Theta \in \widehat{\mathcal{W}}^{\beta} \backslash \widehat{\mathcal{W}}} \widehat{\mathcal{Z}}^{\beta}(\Omega \cap \Theta) .
\end{aligned}
$$

Proof. The inclusion $\widehat{\mathcal{Z}}(\Omega) \subset \bigoplus_{w \in \Omega} \widehat{S}$ yields an inclusion $\widehat{\mathcal{Z}}^{\emptyset}(\Omega) \subset \bigoplus_{w \in \Omega} \widehat{S}^{\emptyset}$. We want to show that this is a bijection. Let $d \in \widehat{S}$ be the product of all $\alpha_{t}$, where $t \in \widehat{\mathcal{T}}$ is a reflection connecting two elements in $\Omega$. From the definition of $\widehat{\mathcal{Z}}(\Omega)$ it is immediate that $\bigoplus_{w \in \widehat{\mathcal{W}}} d \widehat{S} \subset \widehat{\mathcal{Z}}(\Omega)$, and since $d$ is invertible in $\widehat{S}^{\emptyset}$ we can deduce our first claim.

For the second claim we argue analogously. Let us denote by $L$ the set on the right-hand side of the second claimed equation. It is clear that $\widehat{\mathcal{Z}}^{\beta}(\Omega)$ is contained in $L$. Hence it is enough to show that $L \cap \bigoplus_{w \in \Omega} \widehat{S}$ is contained in $\widehat{\mathcal{Z}}^{\beta}(\Omega)$. Now let $d \in \widehat{S}$ be the product of all $\alpha_{t}$ for reflections $t$ that are not of type $\beta$ (i.e. $t \neq s_{\beta, n}$ for all $n$ ), but connect two elements in $\Omega$. Then we have $L \cap \bigoplus_{w \in \Omega} d \widehat{S} \subset \widehat{\mathcal{Z}}(\Omega)$. As $d$ is invertible in $\widehat{S}^{\beta}$, we can deduce the second equation. The third is a direct consequence. 
Let $M \in \mathcal{H}$ and define $M^{\emptyset}:=M \otimes_{\widehat{S}} \widehat{S}^{\emptyset}$ and $M^{\beta}:=M \otimes_{\widehat{S}} \widehat{S}^{\beta}$. Recall that $M$ is a module for $\widehat{\mathcal{Z}}(\Omega)$ for some finite subset $\Omega$ of $\widehat{\mathcal{W}}$ by Lemma 2.5. So $M^{\emptyset}$ and $M^{\beta}$ are modules over $\widehat{\mathcal{Z}}^{\emptyset}(\Omega)$ and $\widehat{\mathcal{Z}}^{\beta}(\Omega)$, respectively.

Lemma 3.2. The decompositions in Lemma 3.1 induce decompositions

$$
M^{\emptyset}=\bigoplus_{w \in \widehat{\mathcal{W}}} M^{\emptyset, w}, \quad M^{\beta}=\bigoplus_{\Theta \in \widehat{\mathcal{W}}^{\beta} \backslash \widehat{\mathcal{W}}} M^{\beta, \Theta} .
$$

Now fix $s \in \widehat{\mathcal{S}}$. We want to prove a similar statement for objects $N \in \mathcal{H}^{s}$. Suppose that $\Omega \subset \widehat{\mathcal{W}}$ is $s$-invariant. Then the involution $\sigma_{s}$ induces an involution on $\widehat{\mathcal{Z}}^{\emptyset}(\Omega)$ and on $\widehat{\mathcal{Z}}^{\beta}(\Omega)$ for any $\beta \in R^{+}$that we denote, for simplicity, by $\sigma_{s}$ as well. We let $\widehat{\mathcal{Z}}^{\emptyset}(\Omega)^{s}$ and $\widehat{\mathcal{Z}}^{\beta}(\Omega)^{s}$ be the corresponding sets of invariants.

Recall that $\widehat{\mathcal{W}}_{s}=\{1, s\}$ is the parabolic subgroup corresponding to $s$ and that $\widehat{\mathcal{W}}^{s}=\widehat{\mathcal{W}} / \widehat{\mathcal{W}}_{s}$. For $w \in \widehat{\mathcal{W}}$ we denote by $\bar{w} \in \widehat{\mathcal{W}}^{s}$ its canonical image. The group $\widehat{\mathcal{W}}^{\beta}$ still acts on $\widehat{\mathcal{W}}^{s}$ from the left and the quotient map $\widehat{\mathcal{W}} \rightarrow \widehat{\mathcal{W}}^{s}$ is $\widehat{\mathcal{W}}^{\beta}$-equivariant. For $\Theta \in \widehat{\mathcal{W}}^{\beta} \backslash \widehat{\mathcal{W}}$ we denote by $\bar{\Theta} \in \widehat{\mathcal{W}}^{\beta} \backslash \widehat{\mathcal{W}}^{s}$ its canonical image. From Lemma 3.1 we get the decompositions

$$
\widehat{\mathcal{Z}}^{\emptyset}(\Omega)^{s}=\bigoplus_{\bar{w} \in \bar{\Omega}} \widehat{S}^{\emptyset}, \quad \widehat{\mathcal{Z}}^{\beta}(\Omega)^{s}=\bigoplus_{\bar{\Theta} \in \widehat{\mathcal{W}}^{\beta} \backslash \widehat{\mathcal{W}}^{s}} \widehat{\mathcal{Z}}^{\beta}(\Omega \cap \bar{\Theta})^{s} .
$$

Hence we obtain the following analog of Lemma 3.2

Lemma 3.3. Let $N \in \mathcal{H}^{s}$. Then there are canonical decompositions

$$
N^{\emptyset}=\bigoplus_{\bar{w} \in \widehat{\mathcal{W}}^{s}} N^{\emptyset, \bar{w}}, \quad N^{\beta}=\bigoplus_{\bar{\Theta} \in \widehat{\mathcal{W}}^{\beta} \backslash \widehat{\mathcal{W}}^{s}} N^{\beta, \bar{\Theta}} .
$$

3.1. Localization of translation functors. Now we study the behaviour of the translation functors under base change. Let us fix $s \in \widehat{\mathcal{S}}$.

Lemma 3.4. For $M \in \mathcal{H}, N \in \mathcal{H}^{s}$ and $w \in \widehat{\mathcal{W}}$ we have

$$
\left(\vartheta_{\text {on }}^{s} M\right)^{\emptyset, \bar{w}}=M^{\emptyset, w} \oplus M^{\emptyset, w s}, \quad\left(\vartheta_{\text {out }}^{s} N\right)^{\emptyset, w}=N^{\emptyset, \bar{w}} .
$$

Proof. Note that for a finite $s$-invariant subset $\Omega$ of $\widehat{\mathcal{W}}$ the inclusion $\widehat{\mathcal{Z}}^{\emptyset}(\Omega)^{s} \subset$ $\widehat{\mathcal{Z}}^{\emptyset}(\Omega)$ equals $\left(\bigoplus_{w \in \Omega} \widehat{S}^{\emptyset}\right)^{s} \subset \bigoplus_{w \in \Omega} \widehat{S}^{\emptyset}$ and the claims follow immediately.

Now fix $\beta \in R^{+}$. For $\Theta \in \widehat{\mathcal{W}}^{\beta} \backslash \widehat{\mathcal{W}}$ we have two cases to distinguish. Either we have $\Theta=\Theta s$ or $\Theta \neq \Theta s$. In the latter case the map that sends $w$ to $\bar{w}$ yields a bijection $\Theta \stackrel{\sim}{\rightarrow} \bar{\Theta}$.

Lemma 3.5. Choose $\Theta \in \widehat{\mathcal{W}}^{\beta} \backslash \widehat{\mathcal{W}}$ and let $\bar{\Theta} \in \widehat{\mathcal{W}}^{\beta} \backslash \widehat{\mathcal{W}}^{s}$ be its image. Then

(1) $\left(\vartheta_{o n}^{s} M\right)^{\beta, \bar{\Theta}}=M^{\beta, \Theta} \oplus M^{\beta, \Theta s}$ if $\Theta \neq \Theta s$, and $\left(\vartheta_{o n}^{s} M\right)^{\beta, \bar{\Theta}}=M^{\beta, \Theta}$ if $\Theta=\Theta s$.

(2) $\left(\vartheta_{\text {out }}^{s} N\right)^{\beta, \Theta}=N^{\beta, \bar{\Theta}}$ if $\Theta \neq \Theta s$, and $\left(\vartheta_{\text {out }}^{s} N\right)^{\beta, \Theta}=\widehat{\mathcal{Z}}^{\beta}(\Theta) \otimes_{\widehat{\mathcal{Z}}^{\beta}(\Theta) s} N^{\beta, \bar{\Theta}}$ if $\Theta=\Theta s$.

Proof. In the case $\Theta=\Theta s$ the inclusion $\widehat{\mathcal{Z}}^{\beta}(\Omega)^{s} \subset \widehat{\mathcal{Z}}^{\beta}(\Omega)$ contains $\widehat{\mathcal{Z}}^{\beta}(\Theta)^{s} \subset$ $\widehat{\mathcal{Z}}^{\beta}(\Theta)$ as a direct summand. In the case $\Theta \neq \Theta s$ we have an analogous direct summand $\widehat{\mathcal{Z}}^{\beta}(\Theta \cup \Theta s)^{s} \subset \widehat{\mathcal{Z}}^{\beta}(\Theta) \oplus \widehat{\mathcal{Z}}^{\beta}(\Theta s)$, and this inclusion is an isomorphism on each direct summand. From these facts the claims follow easily. 
Let $\Omega \subset \widehat{\mathcal{W}}$ be a subset and $\Lambda:=\widehat{\mathcal{W}} \backslash \Omega$ its complement. Let $M$ be an object in $\mathcal{H}$. We consider the canonical inclusion $M \subset M^{\emptyset}=\bigoplus_{w \in \widehat{\mathcal{W}}} M^{\emptyset, w}$ and define

$$
\begin{aligned}
& M_{\Lambda}:=M \cap \bigoplus_{w \in \Lambda} M^{\emptyset, w}, \\
& M^{\Omega}:=M / M_{\Lambda}=\operatorname{im}\left(M \rightarrow M^{\emptyset} \rightarrow \bigoplus_{w \in \Omega} M^{\emptyset, w}\right) .
\end{aligned}
$$

If $\Omega^{\prime} \subset \Omega$ are subsets in $\widehat{\mathcal{W}}$ with complements $\Lambda \subset \Lambda^{\prime}$, then we have an inclusion $M_{\Lambda} \subset M_{\Lambda^{\prime}}$ and a corresponding surjection $M^{\Omega} \rightarrow M^{\Omega^{\prime}}$.

Let $N \in \mathcal{H}^{s}$. For any subset $\bar{\Omega}$ of $\widehat{\mathcal{W}}^{s}$ with complement $\bar{\Lambda}$ we can analogously define a submodule $N_{\bar{\Lambda}}$ and a quotient $N^{\bar{\Omega}}$ of $N$. The following is an immediate consequence of Lemma 3.4 .

Lemma 3.6. Let $s \in \widehat{\mathcal{S}}$ and let $\Omega$ be an s-invariant subset of $\widehat{\mathcal{W}}$ with complement $\Lambda$. Denote by $\bar{\Omega}, \bar{\Lambda} \in \widehat{\mathcal{W}}^{s}$ the images. For $M \in \mathcal{H}$ and $N \in \mathcal{H}^{s}$ we have

$$
\begin{array}{cc}
\left(\vartheta_{\text {on }}^{s} M\right)_{\bar{\Lambda}}=\vartheta_{\text {on }}^{s}\left(M_{\Lambda}\right), & \left(\vartheta_{\text {out }}^{s} N\right)_{\Lambda}=\vartheta_{\text {out }}^{s}\left(N_{\bar{\Lambda}}\right), \\
\left(\vartheta_{\text {on }}^{s} M\right)^{\bar{\Omega}}=\vartheta_{\text {on }}^{s}\left(M^{\Omega}\right), & \left(\vartheta_{\text {out }}^{s} N\right)^{\Omega}=\vartheta_{\text {out }}^{s}\left(N^{\bar{\Omega}}\right) .
\end{array}
$$

\section{Filtrations and characters}

In this section we describe how certain partial orders on the set $\widehat{\mathcal{W}}$ induce functorial filtrations on the objects of $\mathcal{H}$ indexed by $\widehat{\mathcal{W}}$. The subquotients of these filtrations turn out to be graded free $\widehat{S}$-modules of finite rank and hence give rise to the definition of a character map from the Grothendieck group of $\mathcal{H}$ to the free $\mathbb{Z}\left[v, v^{-1}\right]$-module $\mathbf{W}$ with basis $\widehat{\mathcal{W}}$. We are particularly interested in the characters associated to the ordinary Bruhat order " $\leqslant$ " and the "generic" Bruhat order " $\succeq$ " on $\widehat{\mathcal{W}}$ (cf. Sections 4.6 and 4.7).

We identify $\mathbf{W}$ with the $\mathbb{Z}\left[v, v^{-1}\right]$-module underlying both the affine Hecke algebra $\mathbf{H}$ and its periodic module $\mathbf{M}$, and we show that the translation combinatorics on $\mathcal{H}$ forms a categorification of $\mathbf{H}$ via the character associated to " $\leqslant$ " (Lemma 4.5), and of $\mathbf{M}$ via the character associated to " $\succeq$ " (Lemma 4.7).

4.1. Partial orders. Let " $\unlhd$ " be a partial order on the set $\widehat{\mathcal{W}}$. For $x, y \in \widehat{\mathcal{W}}$ denote by $[x, y]=\{z \in \widehat{\mathcal{W}} \mid x \unlhd z \unlhd y\}$ the interval between $x$ and $y$. Suppose that " $\unlhd$ " has the following properties:

(1) The elements $w$ and $t w$ are comparable for all $w \in \widehat{\mathcal{W}}$ and $t \in \widehat{\mathcal{T}}$. The relations between all such pairs $w, t w$ generate the partial order.

(2) We have $[w, w s]=\{w, w s\}$ for all $w \in \widehat{\mathcal{W}}$ and $s \in \widehat{\mathcal{S}}$ such that $w \unlhd w s$.

(3) For $x, y \in \widehat{\mathcal{W}}$ such that $x \unlhd x s$ and $y \unlhd x s$ we have $y s \unlhd x s$. For $x, y \in \widehat{\mathcal{W}}$ such that $x s \unlhd x$ and $x s \unlhd y$ we have $x s \unlhd y s$.

We call a subset $\Omega$ of $\widehat{\mathcal{W}}$ open, if $x \in \Omega, y \in \widehat{\mathcal{W}}$ and $x \unlhd y$ imply $y \in \Omega$. Note that assumption (3) implies that if $\Omega$ is open and if $s \in \widehat{\mathcal{S}}$, then $\Omega \cup \Omega s$ is open as well and that for each $x \in \widehat{\mathcal{W}}$ with $x s \unlhd x$ the sets $\{y \in \widehat{\mathcal{W}} \mid y \unlhd x\}$ and $\{y \in \widehat{\mathcal{W}} \mid x s \unlhd y\}$ are $s$-invariant. For $s \in \widehat{\mathcal{S}}$ each coset in $\widehat{\mathcal{W}}^{s}=\widehat{\mathcal{W}} /\{1, s\}$ has a smallest representative by assumption. Hence comparing these representatives gives us an induced partial order on $\widehat{\mathcal{W}}^{s}$ that we denote by " $\unlhd$ " as well. 
4.2. Local sections of moment graph sheaves. Let $\mathscr{F}$ be an object in $\mathcal{A}$. By Proposition 2.8 we have $\Gamma(\mathscr{F}) \in \mathcal{H}$. For any subset $\Omega$ of $\widehat{\mathcal{W}}$ the restriction map $\Gamma(\mathscr{F}) \rightarrow \Gamma(\Omega, \mathscr{F})$ factors over a map $\Gamma(\mathscr{F})^{\Omega} \rightarrow \Gamma(\Omega, \mathscr{F})$. We now prove that these maps are bijections for open sets $\Omega$.

Lemma 4.1. (1) For a sheaf $\mathscr{F}$ in $\mathcal{A}$ and an open subset $\Omega$ of $\widehat{\mathcal{W}}$ we have $\Gamma(\mathscr{F})^{\Omega}=\Gamma(\Omega, \mathscr{F})$.

(2) For a simple affine reflection $s$, a sheaf $\mathscr{G}$ in $\mathcal{A}^{s}$ and an open subset $\bar{\Omega}$ of $\widehat{\mathcal{W}}^{s}$ we have $\Gamma(\mathscr{G})^{\bar{\Omega}}=\Gamma(\bar{\Omega}, \mathscr{G})$.

Proof. Statement (1) is obvious in the case $\mathscr{F}=\mathscr{B}_{e}$. So using induction it is enough to consider the following situation. Suppose that (1) holds for $\mathscr{F}^{\prime} \in \mathcal{A}$. We then have to show that (2) holds for $\mathscr{G}=\widetilde{\vartheta}_{\text {on }}^{s} \mathscr{F}^{\prime}$ and (1) holds for $\mathscr{F}=\widetilde{\vartheta}_{\text {out }}^{s} \mathscr{G}$. We repeatedly use Lemmas 2.6 and 3.6 in the following.

So suppose that $\bar{\Omega}$ is open in $\widehat{\mathcal{W}}^{s}$. Then its preimage $\Omega$ is $s$-invariant and open in $\widehat{\mathcal{W}}$. We have $\Gamma\left(\widetilde{\vartheta}_{o n}^{s} \mathscr{F}^{\prime}\right)^{\bar{\Omega}}=\left(\vartheta_{o n}^{s} \Gamma\left(\mathscr{F}^{\prime}\right)\right)^{\bar{\Omega}}=\vartheta_{o n}^{s}\left(\Gamma\left(\mathscr{F}^{\prime}\right)^{\Omega}\right)=\vartheta_{o n}^{s}\left(\Gamma\left(\Omega, \mathscr{F}^{\prime}\right)\right)=$ $\Gamma\left(\bar{\Omega}, \widetilde{\vartheta}_{o n}^{s} \mathscr{F}^{\prime}\right)$, and we have shown property (2) for $\mathscr{G}=\widetilde{\vartheta}_{o n}^{s} \mathscr{F}^{\prime}$.

Now consider the sheaf $\mathscr{F}=\widetilde{\vartheta}_{\text {out }}^{s} \mathscr{G}$. Let $\Omega$ be open in $\widehat{\mathcal{W}}$ and suppose first that $\Omega$ is $s$-invariant. Then we have $\Gamma\left(\widetilde{\vartheta}_{\text {out }}^{s} \mathscr{G}\right)^{\Omega}=\left(\vartheta_{\text {out }}^{s} \Gamma(\mathscr{G})\right)^{\Omega}=\vartheta_{\text {out }}^{s}\left(\Gamma(\mathscr{G})^{\bar{\Omega}}\right)=$ $\vartheta_{\text {out }}^{s} \Gamma(\bar{\Omega}, \mathscr{G})=\Gamma\left(\Omega, \widetilde{\vartheta}_{\text {out }}^{s} \mathscr{G}\right)$; hence we proved (1) for $s$-invariant $\Omega$.

Note that the claim in (1) is equivalent to the surjectivity of the restriction $\Gamma(\mathscr{F}) \rightarrow \Gamma(\Omega, \mathscr{F})$. So we have proven this under the $s$-invariance assumption. For an arbitrary open subset $\Omega$, the set $\Omega \cup \Omega s$ is open as well. Using induction it is hence enough to show that $\Gamma(\Omega \cup\{x\}, \mathscr{F}) \rightarrow \Gamma(\Omega, \mathscr{F})$ is surjective for any $x \in \Omega s$, $x \notin \Omega$ such that $\Omega \cup\{x\}$ is open as well. So let us fix such an $x$ and let us choose a section $m=\left(m_{y}\right)_{y \in \Omega}$ in $\Gamma(\Omega, \mathscr{F})$. We want to find some $m_{x} \in \mathscr{F}^{x}$ such that $\left(\left(m_{y}\right)_{y \in \Omega}, m_{x}\right)$ is a section of $\mathscr{F}$ over $\Omega \cup\{x\}$.

Now any two connected vertices in the graph are comparable; hence in order to find $m_{x}$ it suffices to consider only those $m_{y}$ with $x \triangleleft y$. We have $x \triangleleft x s$ and hence the set $\{y \mid x \triangleleft y\} \backslash\{x s\}$ is $s$-invariant. As we have proven the surjectivity statement already for $s$-invariant open sets, we can assume that $m_{y}=0$ for all $y \neq x s$ with $x \triangleleft y$. But in this case it immediately follows from the definition of $\widetilde{\vartheta}_{\text {out }}^{s}$ that setting $m_{x}:=m_{x s}$ actually yields a section $\left((0)_{x \triangleleft y, y \neq x s}, m_{x}, m_{x s}\right)$ of $\mathscr{F}$ over $\{y \mid x \unlhd y\}$, hence an extension of $m$ to the vertex $x$. Hence $\Gamma(\Omega \cup\{x\}, \mathscr{F}) \rightarrow \Gamma(\Omega, \mathscr{F})$ is a surjective map.

4.3. Subquotients. Let $\mathscr{F} \in \mathcal{A}$ and let $M=\Gamma(\mathscr{F}) \in \mathcal{H}$. Let $\Omega \subset \widehat{\mathcal{W}}$ be open and suppose that $x$ is a minimal element in $\Omega$, so $\Omega^{\prime}:=\Omega \backslash\{x\}$ is open as well. We let $K=K_{\Omega, x}$ be the kernel of the surjection $M^{\Omega} \rightarrow M^{\Omega^{\prime}}$. From Lemma4.1 we deduce that $K$ can be identified with the kernel of the restriction map $\Gamma(\Omega, \mathscr{F}) \rightarrow \Gamma\left(\Omega^{\prime}, \mathscr{F}\right)$, hence with the elements in $\Gamma(\Omega, \mathscr{F})$ supported on $\{x\}$. Hence this is the set of elements $m_{x}$ in $\mathscr{F}^{x}$ with the property that $\rho_{x, E}\left(m_{x}\right)=0$ for any edge $E$ connecting $x$ to some $y$ with $x \triangleleft y$. Now the latter description is independent of the choice of $\Omega$ (but certainly depends on " $\triangleleft$ "). Hence we obtain that $M_{[x]}=M_{[x, \unlhd]}:=K_{\Omega, x}$ is a well-defined subquotient of $M$.

Suppose that $\Lambda \subset \widehat{\mathcal{W}}$ is the complement of $\Omega$. Then $\Lambda^{\prime}=\Lambda \cup\{x\}$ is the complement of $\Omega^{\prime}$. Clearly, the cokernel of $M_{\Lambda} \rightarrow M_{\Lambda^{\prime}}$ identifies with the kernel of $M^{\Omega} \rightarrow M^{\Omega^{\prime}}$, hence with $M_{[x]}$. In particular, we can identify $M_{[x]}$ with the image of 
the map $M_{\{y \mid y \unlhd x\}} \subset \bigoplus_{y} M^{\emptyset, y} \rightarrow M^{\emptyset, x}$, where the last map is the projection along the decomposition.

Let $s$ be a simple affine reflection and let $x \in \widehat{\mathcal{W}}$. Then $\{x, x s\}$ is an interval in the partial order " $\unlhd$ ". Suppose that $x \triangleleft x$ s and set $\Omega:=\{y \mid x \unlhd y\}, \Omega^{\prime}:=\Omega \backslash\{x\}$ and $\Omega^{\prime \prime}=\Omega^{\prime} \backslash\{x s\}$. Then $\Omega, \Omega^{\prime}$ and $\Omega^{\prime \prime}$ are open. We let $M_{[x, x s]}$ be the kernel of the surjection $M^{\Omega} \rightarrow M^{\Omega^{\prime \prime}}$. As $M_{[x]}$ and $M_{[x s]}$ can be identified with the kernels of $M^{\Omega} \rightarrow M^{\Omega^{\prime}}$ and $M^{\Omega^{\prime}} \rightarrow M^{\Omega^{\prime \prime}}$ we obtain a short exact sequence

$$
0 \rightarrow M_{[x]} \rightarrow M_{[x, x s]} \rightarrow M_{[x s]} \rightarrow 0 .
$$

Lemma 4.2. Let $x \in \widehat{\mathcal{W}}, s \in \widehat{\mathcal{S}}$ and suppose that $x \unlhd x s$. Then we have isomorphisms

$$
\begin{aligned}
&\left(\theta^{s} M\right)_{[x]} \cong M_{[x]}\langle-2\rangle \oplus M_{[x s]}\langle-2\rangle, \\
&\left(\theta^{s} M\right)_{[x s]} \cong M_{[x]} \oplus M_{[x s]}
\end{aligned}
$$

of graded $\widehat{S}$-modules. In particular, for each $M \in \mathcal{H}$ and all $y \in \widehat{\mathcal{W}}$ the space $M_{[y]}$ is a graded free $\widehat{S}$-module of finite rank.

Proof. Since $\Omega$ and $\Omega^{\prime \prime}$ are $s$-invariant we have, by Lemma 3.6. $\theta^{s} M^{\Omega}=\left(\theta^{s} M\right)^{\Omega}$ and $\theta^{s} M^{\Omega^{\prime \prime}}=\left(\theta^{s} M\right)^{\Omega^{\prime \prime}}$. As $\theta^{s}$ is an exact functor on $\widehat{\mathcal{Z}}$-mod ${ }^{f}$, we can deduce $\theta^{s} M_{[x, x s]}=\left(\theta^{s} M\right)_{[x, x s]}$. Now $\theta^{s} M_{[x, x s]}=\widehat{\mathcal{Z}}(\{x, x s\}) \otimes_{\widehat{\mathcal{Z}}(\{x, x s\})^{s}} M_{[x, x s]}=$ $\widehat{\mathcal{Z}}(\{x, x s\}) \otimes_{\widehat{S}} M_{[x, x s]}$. Moreover, $\widehat{\mathcal{Z}}(\{x, x s\})_{[x]} \cong \widehat{S}\langle-2\rangle$ and $\widehat{\mathcal{Z}}(\{x, x s\})_{[x s]} \cong \widehat{S}$. From the short exact sequence above we deduce that the $\widehat{S}$-modules $M_{[x, x s]}$ and $M_{[x]} \oplus M_{[x s]}$ are isomorphic; hence we get the isomorphisms that are stated in the lemma.

4.4. The $k$-linear version. Let $k$ be a ring in which 2 is invertible. Recall that we defined $\widehat{\mathcal{Z}}_{k}=\widehat{\mathcal{Z}} \otimes_{\mathbb{Z}^{\prime}} k$ and $\widehat{\mathcal{Z}}_{k}^{s}=\widehat{\mathcal{Z}}^{s} \otimes_{\mathbb{Z}^{\prime}} k$; hence any module in $\mathcal{H}_{k}$ occurs as a direct summand of $M_{k}=M \otimes_{\mathbb{Z}^{\prime}} k$ for some object $M$ of $\mathcal{H}$. We now translate the previous results on the category $\mathcal{H}$ to $\mathcal{H}_{k}$ using base change.

For a finite subset $\Omega$ of $\widehat{\mathcal{W}}$ we set $\widehat{\mathcal{Z}}_{k}(\Omega)=\widehat{\mathcal{Z}}(\Omega)_{k}$. If $\Omega$ is $s$-invariant, we set $\widehat{\mathcal{Z}}_{k}(\Omega)^{s}=\left(\widehat{\mathcal{Z}}(\Omega)^{s}\right)_{k}$. We get canonical maps $\widehat{\mathcal{Z}}_{k} \rightarrow \widehat{\mathcal{Z}}_{k}(\Omega), \widehat{\mathcal{Z}}_{k}^{s} \rightarrow \widehat{\mathcal{Z}}_{k}(\Omega)^{s}$ and the statements of Lemma 2.5 carry over to the $k$-linear versions. Now the statement of Lemma 2.6 does not necessarily carry over, as we used an analog of the GKMproperty in its proof (but compare Proposition 9.4), which is why we defined the category $\mathcal{A}$ only over $\mathbb{Z}^{\prime}$.

We set $\widehat{\mathcal{Z}}_{k}^{\emptyset}(\Omega)=\widehat{\mathcal{Z}}^{\emptyset}(\Omega)_{k}$ and $\widehat{\mathcal{Z}}_{k}^{\beta}(\Omega)=\widehat{\mathcal{Z}}^{\beta}(\Omega)_{k}$ for $\beta \in R^{+}$. From Lemma 3.1 we deduce $\widehat{\mathcal{Z}}_{k}^{\emptyset}(\Omega)=\bigoplus_{w \in \Omega} \widehat{S}_{k}^{\emptyset}$ and $\widehat{\mathcal{Z}}_{k}^{\beta}(\Omega)=\bigoplus_{\Theta \in \widehat{\mathcal{W}}^{\beta} \backslash \widehat{\mathcal{W}}^{\prime}} \widehat{\mathcal{Z}}_{k}^{\beta}(\Omega \cap \Theta)$. So we get a decomposition as in Lemma 3.2 also for objects in $\mathcal{H}_{k}$. For a simple affine reflection $s$ and an $s$-invariant $\Omega$ we set $\widehat{\mathcal{Z}}_{k}^{\emptyset}(\Omega)^{s}=\left(\widehat{\mathcal{Z}}^{\emptyset}(\Omega)^{s}\right)_{k}$, etc. Then the statements of Lemma 3.3 carry over. For $M \in \mathcal{H}_{k}$ or $N \in \mathcal{H}_{k}^{s}$ we define $M^{\Omega}, M_{\Lambda}, N^{\bar{\Omega}}$ and $N_{\bar{\Lambda}}$ as before using the generic decompositions. These decompositions are compatible with base change, i.e. for $M \in \mathcal{H}$ and $w \in \widehat{\mathcal{W}}$ we have $M_{k}^{\emptyset, w}=\left(M^{\emptyset, w}\right)_{k}$, etc., so Lemmas 3.4, 3.5 and 3.6 carry over.

Now we define, for $M \in \mathcal{H}_{k}$ and $x \in \widehat{\mathcal{W}}$ the subquotients $M_{[x]}$ and $M_{[x, x s]}$ as the kernels of the maps $M^{\unrhd x} \rightarrow M^{\triangleright x}$ and $M^{\unrhd x} \rightarrow M^{\triangleright x, \neq x s}$ if $x s \triangleright x$. Then the freeness result of Lemma 4.2 implies that for $M \in \mathcal{H}$ we have $\left(M_{k}\right)_{[x]}=\left(M_{[x]}\right)_{k}$, etc. Hence also Lemma 4.2 carries over. 
From now on we consider for a given $M \in \mathcal{H}_{k}$ the quotients $M^{\Omega}$ only for open subsets $\Omega$, and the submodules $M_{\Lambda}$ only for their closed complements $\Lambda$.

4.5. Character maps associated to partial orders. Suppose that $L$ is a $\mathbb{Z}$ graded, graded free $\widehat{S}_{k}$-module of finite rank, i.e. $L \cong \bigoplus_{i=1, \ldots, n} \widehat{S}_{k}\left\langle k_{i}\right\rangle$ for some $k_{i} \in \mathbb{Z}$. The multiset of numbers $\left\{k_{i}\right\}$ is well defined, so we set

$$
\underline{\mathrm{rk}} L:=\sum_{i=1, \ldots, n} v^{-k_{i}} \in \mathbb{N}\left[v, v^{-1}\right] .
$$

Choose a length function $l: \widehat{\mathcal{W}} \rightarrow \mathbb{Z}$ with respect to " $\unlhd$ ", i.e. a function with the property that $l(x s)=l(x)+1$ for all $x \in \widehat{\mathcal{W}}$ and $s \in \widehat{\mathcal{S}}$ with $x \unlhd x s$. Assume that $l(e)=0$. Let $\mathbf{W}$ be the free $\mathbb{Z}\left[v, v^{-1}\right]$-module with basis $\left\{W_{x}\right\}_{x \in \widehat{\mathcal{W}}}$. By Lemma 4.2 we can define for each $M \in \mathcal{H}_{k}$ the element

$$
h_{\unlhd, l}(M):=\sum_{x \in \widehat{\mathcal{W}}} v^{l(x)} \underline{\mathrm{rk}} M_{[x, \unlhd]} W_{x} \in \mathbf{W} .
$$

This yields a map from the Grothendieck group of $\mathcal{H}_{k}$ to $\mathbf{W}$ that we denote as $h_{\unlhd, l}: \mathcal{H}_{k} \rightarrow \mathbf{W}$. For $s \in \widehat{\mathcal{S}}$ we define the $\mathbb{Z}\left[v, v^{-1}\right]$-linear map $\rho_{s, \unlhd}: \mathbf{W} \rightarrow \mathbf{W}$ by

$$
\rho_{s, \unlhd}\left(W_{x}\right):= \begin{cases}W_{x s}+v^{-1} W_{x} & \text { if } x s \unlhd x, \\ W_{x s}+v W_{x} & \text { if } x \unlhd x s .\end{cases}
$$

Proposition 4.3. For each $M \in \mathcal{H}_{k}$ and $s \in \widehat{\mathcal{S}}$ we have

$$
h_{\unlhd, l}\left(\theta^{s} M\langle 1\rangle\right)=\rho_{s, \unlhd}\left(h_{\unlhd, l}(M)\right) .
$$

Proof. For $x \in \widehat{\mathcal{W}}$ we have, by Lemma 4.2,

$$
\underline{\mathrm{rk}}\left(\theta^{s} M\right)_{[x]}= \begin{cases}\underline{\mathrm{rk}} M_{[x]}+\underline{\mathrm{rk}} M_{[x s]} & \text { if } x s \unlhd x, \\ v^{2} \cdot \underline{\mathrm{rk}} M_{[x]}+v^{2} \cdot \underline{\mathrm{rk}} M_{[x s]} & \text { if } x \unlhd x s .\end{cases}
$$

Hence

$$
\begin{aligned}
h_{\unlhd, l}\left(\theta^{s} M\langle 1\rangle\right)= & \sum_{x \in \widehat{\mathcal{W}}} v^{l(x)-1} \underline{\mathrm{rk}}\left(\theta^{s} M\right)_{[x]} W_{x} \\
= & \sum_{x \in \widehat{\mathcal{W}}, x s \unlhd x} v^{l(x)-1}\left(\underline{\mathrm{rk}} M_{[x]}+\underline{\mathrm{rk}} M_{[x s]}\right) W_{x} \\
& +\sum_{x \in \widehat{\mathcal{W}}, x \unlhd x s} v^{l(x)+1}\left(\underline{\mathrm{rk}} M_{[x s]}+\underline{\mathrm{rk}} M_{[x]}\right) W_{x} .
\end{aligned}
$$


Moreover,

$$
\begin{aligned}
\rho_{s, \unlhd}\left(h_{\unlhd}(M)\right)= & \sum_{x \in \widehat{\mathcal{W}}}\left(v^{l(x)} \underline{\mathrm{rk}} M_{[x]}\right) \rho_{s, \unlhd}\left(W_{x}\right) \\
= & \sum_{x \in \widehat{\mathcal{W}}, x s \unlhd x}\left(v^{l(x)} \underline{\mathrm{rk}} M_{[x]}\right)\left(W_{x s}+v^{-1} W_{x}\right) \\
& +\sum_{x \in \widehat{\mathcal{W}}, x \unlhd}\left(v^{l(x)} \underline{\mathrm{rk}} M_{[x]}\right)\left(W_{x s}+v W_{x}\right) \\
= & \sum_{x \in \widehat{\mathcal{W}}, x s \unlhd x}\left(v^{l(x)-1} \underline{\mathrm{rk}} M_{[x]}+v^{l(x)-1} \underline{\mathrm{rk}} M_{[x s]}\right) W_{x} \\
& +\sum_{x \in \widehat{\mathcal{W}}, x \unlhd x s}\left(v^{l(x)+1} \underline{\mathrm{rk}} M_{[x]}+v^{l(x)+1} \underline{\mathrm{rk}} M_{[x s]}\right) W_{x} \\
= & h_{\unlhd, l}\left(\theta^{s} M\langle 1\rangle\right) .
\end{aligned}
$$

Hence the proposition is proven.

In the following we consider more closely the character maps associated to the Bruhat order and the generic Bruhat order.

4.6. The Bruhat order and the affine Hecke algebra. In the following we will denote by " $\leqslant$ " the Bruhat order on $\widehat{\mathcal{W}}$ with respect to $\widehat{\mathcal{S}}$. It has all the properties listed in Section 4.1 (for property (3), see Hum90, Proposition 5.9]). Denote by $l: \widehat{\mathcal{W}} \rightarrow \mathbb{N}$ the associated length function.

Let $\mathbf{H}=\bigoplus_{x \in \widehat{\mathcal{W}}} \mathbb{Z}\left[v, v^{-1}\right] \cdot T_{x}$ be the affine Hecke algebra. Its multiplication is given by the formulas

$$
\begin{aligned}
T_{x} \cdot T_{y} & =T_{x y} \quad \text { if } l(x y)=l(x)+l(y), \\
T_{s}^{2} & =v^{-2} T_{e}+\left(v^{-2}-1\right) T_{s} \quad \text { for } s \in \widehat{\mathcal{S}} .
\end{aligned}
$$

Then $T_{e}$ is a unit in $\mathbf{H}$ and for any $x \in \widehat{\mathcal{W}}$ there exists an inverse of $T_{x}$ in $\mathbf{H}$. For $s \in \widehat{\mathcal{S}}$ we have $T_{s}^{-1}=v^{2} T_{s}+\left(v^{2}-1\right)$. There is a duality (i.e. a $\mathbb{Z}$-linear involution) $d: \mathbf{H} \rightarrow \mathbf{H}$, given by $d(v)=v^{-1}$ and $d\left(T_{x}\right)=T_{x^{-1}}^{-1}$ for $x \in \widehat{\mathcal{W}}$.

Set $\tilde{T}_{x}:=v^{l(x)} T_{x}$. Recall the following result:

Theorem 4.4 (KL79, Soe97). For any $x \in \widehat{\mathcal{W}}$ there exists a unique element $\underline{H}_{x}=\sum_{y \in \widehat{\mathcal{W}}} h_{y, x}(v) \cdot \tilde{T}_{y} \in \mathbf{H}$ with the following properties:

(1) $\underline{H}_{x}$ is self-dual, i.e. $d\left(\underline{H}_{x}\right)=\underline{H}_{x}$,

(2) $h_{y, x}(v)=0$ if $y \not \leq x$, and $h_{x, x}(v)=1$,

(3) $h_{y, x}(v) \in v \mathbb{Z}[v]$ for $y<x$.

For example, we have $\underline{H}_{e}=\tilde{T}_{e}$ and $\underline{H}_{s}=\tilde{T}_{s}+v \tilde{T}_{e}$ for each $s \in \widehat{\mathcal{S}}$.

Now we identify the free $\mathbb{Z}\left[v, v^{-1}\right]$-modules $\mathbf{H}$ and $\mathbf{W}$ by means of the map $\tilde{T}_{x} \mapsto W_{x}$ for all $x \in \widehat{\mathcal{W}}$. In the following we will consider $h_{\leqslant, l}$ as a character map from $\mathcal{H}_{k}$ to $\mathbf{H}$. A short and simple calculation yields the following result.

Lemma 4.5. Choose $s \in \widehat{\mathcal{S}}$. Under the above identification the right multiplication with $\underline{H}_{s}$ on $\mathbf{H}$ corresponds to the map $\rho_{s, \leqslant}: \mathbf{H} \rightarrow \mathbf{H}$. Hence

$$
h_{\leqslant, l}\left(\theta^{s} M\langle 1\rangle\right)=h_{\leqslant, l}(M) \cdot \underline{H}_{s}
$$

for all $M \in \mathcal{H}_{k}$ 
4.7. The generic Bruhat order and the periodic Hecke module. Denote by " $\succeq$ " the generic Bruhat order on $\widehat{\mathcal{W}}$. It can be defined as follows. For $x, y \in \widehat{\mathcal{W}}$ set $x \succeq y$ if $t_{\lambda} y \leqslant t_{\lambda} x$ for sufficiently large $\lambda \in \mathbb{N} R^{\vee,+}$. This order has all the properties we listed at the beginning of this section (for property (3), see Lus80a, Proposition $3.2])$. Let $\delta: \widehat{\mathcal{W}} \rightarrow \mathbb{Z}$ be a length function, normalized such that $\delta(e)=0$.

Let $\mathbf{M}$ be the free $\mathbb{Z}\left[v, v^{-1}\right]$-module with basis $\left\{A_{x}\right\}_{x \in \widehat{\mathcal{W}}}$, and identify $\mathbf{M}$ with W by means of the map $A_{x} \mapsto W_{x}$.

Theorem 4.6 (Soe97]). There is a unique right $\mathbf{H}$-module structure on $\mathbf{M}$, denoted $b y *: \mathbf{M} \times \mathbf{H} \rightarrow \mathbf{M}$, such that for all $s \in \widehat{\mathcal{S}}$ the right multiplication with $\underline{H_{s}}$ is given by the $\operatorname{map} \rho_{s, \succeq}: \mathbf{M} \rightarrow \mathbf{M}$, i.e.

$$
X * \underline{H_{s}}=\rho_{s, \succeq}(X)
$$

for all $X \in \mathbf{M}$ and $s \in \widehat{\mathcal{S}}$.

In the following we will consider $h_{\succeq, \delta}$ as a character map from $\mathcal{H}_{k}$ to $\mathbf{M}$. From Proposition 4.3 and Theorem 4.6 we deduce the following:

Lemma 4.7. For all $M \in \mathcal{H}_{k}$ we have

$$
h_{\succeq, \delta}\left(\theta^{s} M\langle 1\rangle\right)=h_{\succeq, \delta}(M) * \underline{H}_{s} .
$$

The following theorem is part of our main result.

Theorem 4.8. The following diagram commutes:

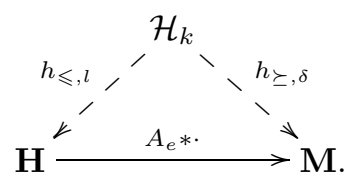

Proof. We have $h_{\succeq, \delta}\left(B_{e}\right)=A_{e}=A_{e} * \underline{H}_{e}=A_{e} * h_{\leqslant, l}\left(B_{e}\right)$. Suppose that $h_{\succeq, \delta}(M)=$ $A_{e} * h_{\leqslant, l}(M)$ for $M \in \mathcal{H}_{k}$ and choose $s \in \widehat{\mathcal{S}}$. Then Lemma 4.5 and Lemma 4.7yield

$$
\begin{aligned}
h_{\succeq, \delta}\left(\theta^{s} M\langle 1\rangle\right) & =h_{\succeq, \delta}(M) * \underline{H}_{s} \\
& =\left(A_{e} * h_{\leqslant, l}(M)\right) * \underline{H}_{s} \\
& \left.=A_{e} *\left(h_{\leqslant, l}(M)\right) \cdot \underline{H}_{s}\right) \\
& =A_{e} * h_{\leqslant, l}\left(\theta^{s} M\langle 1\rangle\right) .
\end{aligned}
$$

The theorem follows by induction.

\section{The Category of Andersen, Jantzen and Soergel}

In this section we define the combinatorial category $\mathcal{M}$ that appears in the work of Andersen, Jantzen and Soergel on Lusztig's conjecture (cf. [AJS94, Soe95]). Its definition is very similar to the definition of $\mathcal{H}$ : It is constructed by applying translation functors to a unit object $P_{0}$ inside a certain category $\mathcal{K}$. We construct a functor $\Psi: \mathcal{H} \rightarrow \mathcal{K}$ with $\Psi\left(B_{e}\right) \cong P_{0}$ and show that $\Psi$ intertwines the translation functors on both sides. From this we deduce that the image of $\Psi$ is contained in $\mathcal{M} \subset \mathcal{K}$.

The translation functors that we define on $\mathcal{K}$ differ from those used in AJS94. In Section 6 we construct an autoequivalence on $\mathcal{K}$ that intertwines both sets of translation functors (Proposition 6.3); hence both sets lead to equivalent categories of special objects. 
5.1. Alcoves and walls. Note that in the constructions so far, the affine Weyl group $\widehat{\mathcal{W}}$ appears in many respects merely as an index set (more precisely, as an index set acted upon by a set of involutions $\widehat{\mathcal{S}}$ ). For the following it is convenient to replace $\widehat{\mathcal{W}}$ by the set $\mathscr{A}$ of alcoves for the affine action of $\widehat{\mathcal{W}}$ on $V^{*}$. So before we state the definition of $\mathcal{K}$, we collect some basic facts and notions from alcove geometry.

For $\alpha \in R^{+}$and $n \in \mathbb{Z}$ define

$$
H_{\alpha, n}^{+}:=\left\{v \in V^{*} \mid\langle\alpha, v\rangle>n\right\}, \quad H_{\alpha, n}^{-}:=\left\{v \in V^{*} \mid\langle\alpha, v\rangle<n\right\} .
$$

Then $V^{*}=H_{\alpha, n}^{-} \dot{\cup} H_{\alpha, n} \dot{\cup} H_{\alpha, n}^{+}$. Consider the coarsest partition of $V^{*}$ that refines the set of all partitions thus obtained. The components of this partition are called facets. Let $\mathscr{F}$ be the set of all facets. Facets that are open in $V^{*}$ are called alcoves. Let $\mathscr{A} \subset \mathscr{F}$ be the set of alcoves. A facet $B$ that is a subset of exactly one hyperplane $H_{\alpha, n}$ is called a wall. We set $\alpha_{B}:=\alpha$ in this case and call $\alpha_{B}$ the type of $B$. Let $\mathscr{W} \subset \mathscr{F}$ be the set of walls. A wall that is a subset of the closure of an alcove $A$ is called a wall of $A$.

Let $C=\left\{v \in V^{*} \mid\langle\alpha, v\rangle>0\right.$ for all $\left.\alpha \in R^{+}\right\}$be the dominant chamber, and denote by $A_{e} \subset C$ the fundamental dominant alcove, i.e. the unique alcove in $C$ whose closure contains the origin 0 of $V^{*}$.

The affine Weyl group acts on $\mathscr{F}$ and leaves the subsets $\mathscr{A}$ and $\mathscr{W}$ stable. This induces a bijection

$$
\begin{aligned}
\widehat{\mathcal{W}} & \stackrel{\sim}{\longrightarrow} \mathscr{A}, \\
w & \mapsto A_{w}:=w\left(A_{e}\right)
\end{aligned}
$$

whose inverse we denote by $A \mapsto w_{A}$. By transport of structure we get a right action of $\widehat{\mathcal{W}}$ on $\mathscr{A}$. It is given explicitly by $\left(A_{w}\right) \cdot x=A_{w x}$.

The walls of $A_{e}$ form a fundamental domain for the $\widehat{\mathcal{W}}$-orbits in $\mathscr{W}$. Moreover, the walls of $A_{e}$ are naturally in bijection with the simple affine reflections $\widehat{\mathcal{S}}$ : associate to $s \in \widehat{\mathcal{S}}$ the unique wall $A_{e, s}$ of $A_{e}$ that lies in the closure of $s . A_{e}$ (this is the wall stabilized by $s$ ). Denote by $\mathscr{A}^{s} \subset \mathscr{W}$ its $\widehat{\mathcal{W}}$-orbit. We get another bijection

$$
\begin{aligned}
\widehat{\mathcal{W}}^{s}=\widehat{\mathcal{W}} /\{1, s\} & \stackrel{\sim}{\rightarrow} \mathscr{A}^{s}, \\
\bar{w} & \mapsto \bar{w}\left(A_{e, s}\right) .
\end{aligned}
$$

We denote by

$$
\begin{aligned}
\mathscr{A} & \rightarrow \mathscr{A}^{s}, \\
A & \mapsto \bar{A}
\end{aligned}
$$

the unique $\widehat{\mathcal{W}}$-equivariant map that sends the alcove $A_{e}$ to its wall $A_{e, s}$, and hence each alcove $A$ to its unique wall $\bar{A}$ in $\mathscr{A}^{s}$. So the diagram



commutes. We define two maps that are splittings of $\mathscr{A} \rightarrow \mathscr{A}^{s}$. For $B \in \mathscr{A}^{s}$ we denote by $B_{-}$and $B_{+}$the unique alcoves with wall $B$ such that $B_{-} \subset H_{\alpha, n}^{-}$and $B_{+} \subset H_{\alpha, n}^{+}$, where $H_{\alpha, n}$ is the hyperplane containing $B$. 
Now choose $\beta \in R^{+}$. For a facet $F \in \mathscr{F}$ define $\beta \uparrow F:=s_{\beta, n} . F$, where $n \in \mathbb{Z}$ is the smallest integer such that $F$ lies on or below $H_{\beta, n}$ (i.e. in $H_{\beta, n}^{-} \cup H_{\beta, n}$ ). Then $\beta \uparrow \cdot: \mathscr{F} \rightarrow \mathscr{F}$ is a bijection whose inverse we denote by $\beta \downarrow \cdot$ Under our identification $\widehat{\mathcal{W}} \cong \mathscr{A}$, an equivalence class of alcoves under the relation generated by $\beta \uparrow \cdot$ corresponds to a coset under the left action of the subgroup $\widehat{\mathcal{W}}^{\beta}$ that is generated by all $s_{\beta, n}$ with $n \in \mathbb{Z}$.

In the following we list some statements which are easy to prove.

Lemma 5.1. Let $s \in \widehat{\mathcal{S}}$ and $\beta \in R^{+}$. Choose $A \in \mathscr{A}$ and $B \in \mathscr{A}^{s}$.

(1) If $\beta \uparrow B=B$, then $\beta \uparrow B_{-}=B_{+}$.

(2) If $\beta \uparrow B \neq B$, then $\left\{\beta \uparrow B_{-}, \beta \uparrow B_{+}\right\}=\left\{(\beta \uparrow B)_{-},(\beta \uparrow B)_{+}\right\}$.

(3) If $\beta \uparrow \bar{A}=\bar{A}$ and $A=\bar{A}_{-}$, then $\overline{\beta \uparrow A}=\bar{A}$ and $\beta \uparrow A=\bar{A}_{+}$.

(4) If $\beta \uparrow \bar{A} \neq \bar{A}$, then $\overline{\beta \uparrow A}=\beta \uparrow \bar{A}$.

5.2. The category of Andersen, Jantzen and Soergel. Let $k$ be a field of characteristic $\neq 2$, and suppose that its characteristic also differs from 3 if $R$ is of type $G_{2}$. Recall that we defined $S_{k}$ as the symmetric algebra $S\left(X \otimes_{\mathbb{Z}} k\right)$ over the $k$-vector space associated to $X$. We define the following sub- $S_{k}$-algebras of the quotient field of $S_{k}$ : Set

$$
S_{k}^{\emptyset}:=S_{k}\left[\alpha^{-1} \mid \alpha \in R^{+}\right] \text {and } S_{k}^{\beta}:=S_{k}\left[\alpha^{-1} \mid \alpha \in R^{+}, \alpha \neq \beta\right]
$$

for $\beta \in R^{+}$. These algebras naturally inherit a $\mathbb{Z}$-grading. For an $S_{k}$-module $M$ set $M^{\beta}:=M \otimes_{S_{k}} S_{k}^{\beta}$ and $M^{\emptyset}:=M \otimes_{S_{k}} S_{k}^{\emptyset}$.

Definition 5.2 (AJS94, Soe95). For a $\widehat{\mathcal{W}}$-orbit $\mathscr{O} \subset \mathscr{F}$ of facets let $\mathcal{K}_{k}(\mathscr{O})$ be the category that consists of objects $M=\left(\{M(F)\}_{F \in \mathscr{O}},\{M(F, \beta)\}_{F \in \mathscr{O}, \beta \in R^{+}}\right)$, where

(1) $M(F)$ is an $S_{k}^{\emptyset}$-module for each $F \in \mathscr{O}$ and

(2) for $F \in \mathscr{O}$ and $\beta \in R^{+}, M(F, \beta)$ is an $S_{k}^{\beta}$-submodule of $M(F) \oplus M(\beta \uparrow F)$ if $\beta \uparrow F \neq F$, and of $M(F)$ if $\beta \uparrow F=F$.

A morphism $f: M \rightarrow N$ in $\mathcal{K}_{k}(\mathscr{O})$ is given by a collection $\left(f_{F}\right)_{F \in \mathscr{O}}$ of homomorphisms $f_{F}: M(F) \rightarrow N(F)$ of $S_{k}^{\emptyset}$-modules, such that for all $F \in \mathscr{O}$ and $\beta \in R^{+}$, $f_{F} \oplus f_{\beta \uparrow F}\left(f_{F}\right.$, resp. $)$ maps $M(F, \beta)$ into $N(F, \beta)$.

We define a degree shift functor $\langle n\rangle: \mathcal{K}_{k}(\mathscr{O}) \rightarrow \mathcal{K}_{k}(\mathscr{O})$ for each $n \in \mathbb{Z}$ by applying the degree shift to all constituents $M(F)$ and $M(F, \beta)$ for $M \in \mathcal{K}_{k}(\mathscr{O})$.

5.3. Translation functors on $\mathcal{K}$. We fix a simple reflection $s \in \widehat{\mathcal{S}}$ and write $\mathcal{K}_{k}$ for $\mathcal{K}_{k}(\mathscr{A})$ and $\mathcal{K}_{k}^{s}$ for $\mathcal{K}_{k}\left(\mathscr{A}^{s}\right)$. In this section we define translation functors $\mathcal{T}_{\text {on }}=\mathcal{T}_{\text {on }}^{s}: \mathcal{K}_{k} \rightarrow \mathcal{K}_{k}^{s}$ and $\mathcal{T}_{\text {out }}=\mathcal{T}_{\text {out }}^{s}: \mathcal{K}_{k}^{s} \rightarrow \mathcal{K}_{k}$. Recall that for an alcove $A$ we denote by $\bar{A}$ its unique wall that lies in the $\widehat{\mathcal{W}}$-orbit $\mathscr{A}^{s}$, and to each wall $B \in \mathscr{A}^{s}$ we associate the neighbouring alcoves $B_{-}$and $B_{+}$.

For $M \in \mathcal{K}_{k}, B \in \mathscr{A}^{s}$ and $\beta \in R^{+}$set

$$
\begin{aligned}
\mathcal{T}_{\text {on }} M(B) & :=M\left(B_{-}\right) \oplus M\left(B_{+}\right), \\
\mathcal{T}_{\text {on }} M(B, \beta) & := \begin{cases}M\left(B_{-}, \beta\right) & \text { if } \beta \uparrow B=B, \\
M\left(B_{-}, \beta\right) \oplus M\left(B_{+}, \beta\right) & \text { if } \beta \uparrow B \neq B .\end{cases}
\end{aligned}
$$

If $\beta \uparrow B=B$ we have $\beta \uparrow B_{-}=B_{+}$, and if $\beta \uparrow B \neq B$ we have $\left\{(\beta \uparrow B)_{-},(\beta \uparrow\right.$ $\left.B)_{+}\right\}=\left\{\beta \uparrow B_{-}, \beta \uparrow B_{+}\right\}$. Hence we can naturally view $\mathcal{T}_{\text {on }} M(B, \beta)$ as a subspace in $\mathcal{T}_{\text {on }} M(B)$ or $\mathcal{T}_{\text {on }} M(B) \oplus \mathcal{T}_{\text {on }} M(\beta \uparrow B)$, respectively. 
For $N \in \mathcal{K}_{k}^{s}, A \in \mathscr{A}$ and $\beta \in R^{+}$set

$$
\begin{aligned}
\mathcal{T}_{\text {out }} N(A):= & N(\bar{A}), \\
\mathcal{T}_{\text {out }} N(A, \beta):= & \begin{cases}\{(\beta x+y, y) \mid x, y \in N(\bar{A}, \beta)\} & \text { if } \beta \uparrow \bar{A}=\bar{A} \\
\beta \cdot N(\bar{A}, \beta) \oplus N(\overline{\beta \uparrow A}, \beta) & \text { and } A=\bar{A}_{-}, \\
N(\bar{A}, \beta) & \text { if } \beta \uparrow \bar{A}=\bar{A} \\
\text { and } A=\bar{A}_{+}, & \text {if } \beta \uparrow \bar{A} \neq \bar{A} .\end{cases}
\end{aligned}
$$

If $\beta \uparrow \bar{A}=\bar{A}$ and $A=\bar{A}_{-}$, then $\overline{\beta \uparrow A}=\bar{A}$. If $\beta \uparrow \bar{A} \neq \bar{A}$, then $\overline{\beta \uparrow A}=\beta \uparrow \bar{A}$. Hence $\mathcal{T}_{\text {out }} N(A, \beta)$ is naturally a subspace in $\mathcal{T}_{\text {out }} N(A) \oplus \mathcal{T}_{\text {out }} N(\beta \uparrow A)$ in each case. We denote by $\mathcal{T}^{s}:=\mathcal{T}_{\text {out }}^{s} \circ \mathcal{T}_{\text {on }}^{s}: \mathcal{K}_{k} \rightarrow \mathcal{K}_{k}$ the composition.

5.4. Special objects in $\mathcal{K}_{k}$. Let $P_{0} \in \mathcal{K}_{k}$ be the object defined by

$$
\begin{aligned}
P_{0}(A) & := \begin{cases}S_{k}^{\emptyset} & \text { if } A=A_{e}, \\
0 & \text { else, }\end{cases} \\
P_{0}(A, \beta) & := \begin{cases}S_{k}^{\beta} & \text { if } A=A_{e} \text { or } A=\beta \downarrow A_{e}, \\
0 & \text { else, }\end{cases}
\end{aligned}
$$

with the obvious inclusions $P_{0}(A, \beta) \subset P_{0}(A) \oplus P_{0}(\beta \uparrow A)$ for all $A \in \mathscr{A}$ and $\beta \in R^{+}$.

Definition 5.3. (1) The category of special objects in $\mathcal{K}_{k}$ is the full subcategory $\mathcal{M}_{k}$ of $\mathcal{K}_{k}$ that consists of all objects that are isomorphic to a direct summand of a direct sum of objects of the form $\mathcal{T}^{s} \circ \cdots \circ \mathcal{T}^{t}\left(P_{0}\right)\langle n\rangle$ for arbitrary sequences $s, \ldots, t \in \widehat{\mathcal{S}}$ and $n \in \mathbb{Z}$.

(2) Let $s \in \widehat{\mathcal{S}}$. The category of special objects in $\mathcal{K}_{k}^{s}$ is the full subcategory $\mathcal{M}_{k}^{s}$ of $\mathcal{K}_{k}^{s}$ that consists of all objects that are isomorphic to a direct summand of $\mathcal{T}_{\text {on }}^{s}(M)$ for some $M \in \mathcal{M}_{k}$.

By definition, the translation functors induce functors $\mathcal{T}_{\text {on }}^{s}: \mathcal{M}_{k} \rightarrow \mathcal{M}_{k}^{s}, \mathcal{T}_{\text {out }}^{s}: \mathcal{M}_{k}^{s}$ $\rightarrow \mathcal{M}_{k}$ and $\mathcal{T}^{s}: \mathcal{M}_{k} \rightarrow \mathcal{M}_{k}$.

5.5. The functor $\Psi$. Recall that $\mathcal{H}_{k}$ is an $\widehat{S}_{k}$-linear category, while $\mathcal{M}_{k}$ is an $S_{k}$-linear category. In order to construct a functor $\Psi: \mathcal{H}_{k} \rightarrow \mathcal{M}_{k}$, we need the homomorphism $\widehat{S}_{k} \rightarrow S_{k}$ of algebras that is induced by the projection ${ }^{-}: \widehat{X}=$ $X \oplus \mathbb{Z} \delta \rightarrow X$ onto the first direct summand.

In Section $\left[3\right.$ we defined the extension $\widehat{S}_{k}^{\emptyset}$ of $\widehat{S}_{k}$ by inverting all affine roots $\alpha_{n}$. Since $\bar{\alpha}_{n}=\alpha$ we get an induced map $\widehat{S}_{k}^{\emptyset} \rightarrow S_{k}^{\emptyset}$. Analogously, we get a map $\widehat{S}_{k}^{\beta} \rightarrow S_{k}^{\beta}$ for each $\beta \in R^{+}$. For an $\widehat{S}_{k}$-module $M$ set $\bar{M}:=M \otimes_{\widehat{S}_{k}} S_{k}$. If $M$ was an $\widehat{S}_{k}^{\beta}$-module (an $\widehat{S}_{k}^{\emptyset}$-module), then $\bar{M}$ is an $S_{k}^{\beta}$-module (an $S_{k}^{\emptyset}$-module, resp.).

In Section 4 we constructed for each $M \in \mathcal{H}_{k}$ a generic decomposition, i.e. a canonical decomposition $M^{\emptyset}=\bigoplus_{w \in \widehat{\mathcal{W}}} M^{\emptyset, w}$. Now we replace $\widehat{\mathcal{W}}$, as an index set, by the set $\mathscr{A}$ using the identification $\widehat{\mathcal{W}} \stackrel{\sim}{\longrightarrow} \mathscr{A}, w \mapsto A_{w}=w\left(A_{e}\right)$. Recall that by " $\succeq$ " we denote the generic Bruhat order on $\widehat{\mathcal{W}}$ that we now consider as a partial order on $\mathscr{A}$. The constructions in Section 4 hence give us a functorial filtration on $\mathcal{H}_{k}$ by the partially ordered set $(\mathscr{A}, \succeq)$; i.e., for each $M \in \mathcal{H}_{k}$ and $A \in \mathscr{A}$ we have the submodule $M_{\succeq A}=M_{\{D \in \mathscr{A} \mid D \succeq A\}} \subset M$. Likewise, we replace $\widehat{\mathcal{W}}^{s}$ by $\mathscr{A}^{s}$ for 
each $s \in \widehat{\mathcal{S}}$, so we get a functorial filtration on $\mathcal{H}_{k}^{s}$ indexed by the partially ordered set $\left(\mathscr{A}^{s}, \succeq\right)$.

Now we define the functor $\Psi: \mathcal{H}_{k} \rightarrow \mathcal{K}_{k}$. For $M \in \mathcal{H}_{k}, A \in \mathscr{A}$ and $\beta \in R^{+}$ define

$$
\begin{aligned}
\Psi M(A) & =\overline{M^{\emptyset, A}} \\
\Psi M(A, \beta) & =\operatorname{im}\left(\overline{M_{\succeq A}^{\beta}} \rightarrow \overline{M^{\emptyset, A}} \oplus \overline{M^{\emptyset, \beta \uparrow A}}\right) .
\end{aligned}
$$

Here the map $\overline{M_{\succeq A}^{\beta}} \rightarrow \overline{M^{\emptyset, A}} \oplus \overline{M^{\emptyset, \beta \uparrow A}}$ is induced by the map

$$
M_{\succeq A}^{\beta} \subset \bigoplus_{B \succeq A} M^{\emptyset, B} \rightarrow M^{\emptyset, A} \oplus M^{\emptyset, \beta \uparrow A},
$$

where the last map is the projection along the decomposition.

For $s \in \widehat{\mathcal{S}}$ we define $\Psi^{s}: \mathcal{H}_{k}^{s} \rightarrow \mathcal{K}_{k}^{s}$ as follows. For $N \in \mathcal{H}_{k}^{s}, B \in \mathscr{A}^{s}$ and $\beta \in R^{+}$ set

$$
\begin{aligned}
\Psi^{s} N(B) & =\overline{N^{\emptyset, B}} \\
\Psi^{s} N(B, \beta) & = \begin{cases}\operatorname{im}\left(\overline{N_{\succeq B}^{\beta}} \rightarrow \overline{N^{\emptyset, B}} \oplus \overline{N^{\emptyset, \beta \uparrow B}}\right) & \text { if } \beta \uparrow B \neq B, \\
\operatorname{im}\left(\overline{N_{\succeq B}^{\beta}} \rightarrow \overline{N^{\emptyset, B}}\right) & \text { if } \beta \uparrow B=B .\end{cases}
\end{aligned}
$$

Again the maps that occur in the definition above are to be understood as the projections along the canonical decomposition. The following theorem provides the most important step in the proof of Theorem 1.1

Theorem 5.4. (1) For $M \in \mathcal{H}_{k}$ we have $\Psi M \in \mathcal{M}_{k}$.

(2) For $M \in \mathcal{H}_{k}$ we have $\operatorname{rk} \Psi M(A)=\operatorname{rk} M^{\emptyset, A}$ for each alcove $A$.

(3) The character map $h_{\succeq, \delta}: \mathcal{H}_{k}-\rightarrow \mathbf{M}$ factors over the functor $\Psi$ and we get a commutative diagram



(4) Let $s \in \widehat{\mathcal{S}}$. For $N \in \mathcal{H}_{k}^{s}$ we have $\Psi^{s} N \in \mathcal{M}_{k}^{s}$ and the following diagrams commute:
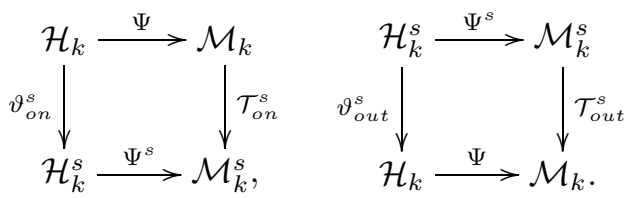

Remark 5.5. In (2), rk denotes the rank of an $S_{k}^{\emptyset}$ or $\widehat{S}_{k}^{\emptyset}$-module, respectively.

Proof. One immediately checks that $\Psi\left(B_{e}\right) \cong P_{0}$. So in order to prove claim (1) and claim (4) we only have to prove that $\Psi$ and $\Psi^{s}$ intertwine the translation functors. This we postpone for a moment.

The claim (2) follows immediately from the definition. Assume that (1) and (4) are proven. Recall that in Section 4.3 we defined for $M \in \mathcal{H}_{k}$ the subquotient $M_{[A, \succeq]}=\operatorname{im}\left(M_{\succeq A} \rightarrow M^{\emptyset, A}\right)$. Now

$$
\overline{M_{[A, \succeq]}^{\beta}}=\operatorname{im}(\Psi(M)(A, \beta) \rightarrow \Psi(M)(A)) .
$$


Hence we can recover $\overline{M_{[A, \succeq]}^{\beta}}$ from the object $\Psi(M)$. Now recall that $M_{[A, \succeq]}$ is a graded free $\widehat{S}_{k}$-module (by Lemma 4.2$)$, so $\overline{M_{[A, \succeq]}}$ is a graded free $S_{k}$-module of the same rank. But we can recover the latter module from the collection $\left\{\overline{M_{[A, \succeq]}^{\beta}}\right\}_{\beta \in R^{+}}$ (as $S_{k}=\bigcap_{\beta \in R^{+}} S_{k}^{\beta}$; cf. AJS94, Lemma 9.1]), hence from $\Psi(M)$, and we can deduce the first part of (3). The second part is then a consequence of Theorem 4.8 .

Now we prove that $\Psi$ and $\Psi^{s}$ intertwine the translation functors. Fix $M \in \mathcal{H}_{k}$, $N \in \mathcal{H}_{k}^{s}, A \in \mathscr{A}$ and $B \in \mathscr{A}^{s}$. By Lemma 3.4 we have

$$
\begin{aligned}
& \left(\vartheta_{\text {on }} M\right)^{\emptyset, B}=M^{\emptyset, B_{-}} \oplus M^{\emptyset, B_{+}}, \\
& \left(\vartheta_{\text {out }} N\right)^{\emptyset, A}=N^{\emptyset, \bar{A}} .
\end{aligned}
$$

Hence

$$
\begin{aligned}
\Psi^{s}\left(\vartheta_{\text {on }} M\right)(B) & =\mathcal{T}_{\text {on }}(\Psi M)(B), \\
\Psi\left(\vartheta_{\text {out }} N\right)(A) & =\mathcal{T}_{\text {out }}\left(\Psi^{s} N\right)(A),
\end{aligned}
$$

which proves already the generic part of the equations $\Psi^{s} \circ \vartheta_{\text {on }}=\mathcal{T}_{\text {on }} \circ \Psi$ and $\Psi \circ \vartheta_{\text {out }}=\mathcal{T}_{\text {out }} \circ \Psi^{s}$. In the following we fix $\beta \in R^{+}$and determine $\Psi \vartheta_{\text {out }} N(A, \beta)$ and $\Psi^{s} \vartheta_{o n} M(B, \beta)$. We start with $\Psi^{s} \vartheta_{o n} M(B, \beta)$.

The case $\beta \uparrow B \neq B$ : We want to determine

$$
\operatorname{im}\left(\left(\vartheta_{o n} M\right)_{\succeq B}^{\beta} \rightarrow\left(\vartheta_{o n} M\right)^{\emptyset, B} \oplus\left(\vartheta_{o n} M\right)^{\emptyset, \beta \uparrow B}\right) .
$$

Since the preimage of the set $\left\{C \in \mathscr{A}^{s} \mid C \succeq B\right\}$ under the map $\mathscr{A} \rightarrow \mathscr{A}^{s}$ is $\left\{D \in \mathscr{A} \mid D \succeq B_{-}\right\}$we have

$$
\left(\vartheta_{o n} M\right)_{\succeq B}^{\beta}=M_{\succeq B_{-}}^{\beta} .
$$

We have already shown that

$$
\left(\vartheta_{\text {on }} M\right)^{\emptyset, B}=M^{\emptyset, B_{-}} \oplus M^{\emptyset, B_{+}}
$$

and

$$
\left(\vartheta_{o n} M\right)^{\emptyset, \beta \uparrow B}=M^{\emptyset,(\beta \uparrow B)_{-}} \oplus M^{\emptyset,(\beta \uparrow B)_{+}}=M^{\emptyset, \beta \uparrow B_{-}} \oplus M^{\emptyset, \beta \uparrow B_{+}}
$$

(for the last identity we used Lemma 5.1). Hence we want to determine

$$
\operatorname{im}\left(M_{\succeq B_{-}}^{\beta} \rightarrow M^{\emptyset, B_{-}} \oplus M^{\emptyset, \beta \uparrow B_{-}} \oplus M^{\emptyset, B_{+}} \oplus M^{\emptyset, \beta \uparrow B_{+}}\right) .
$$

By Lemma 3.2 we have a canonical decomposition

$$
M^{\beta}=\bigoplus_{\Theta \in \widehat{\mathcal{W}}^{\beta} \backslash \mathscr{A}} M^{\beta, \Theta},
$$

where $M^{\beta, \Theta} \subset M^{\beta}$ is the direct summand supported on $\Theta$. The alcoves $B_{-}$and $B_{+}$lie in different $\widehat{\mathcal{W}}^{\beta}$-orbits, and hence the image above splits into the direct sum of

and

$$
\operatorname{im}\left(M_{\succeq B_{-}}^{\beta} \rightarrow M^{\emptyset, B_{-}} \oplus M^{\emptyset, \beta \uparrow B_{-}}\right)
$$

$$
\operatorname{im}\left(M_{\succeq B_{+}}^{\beta} \rightarrow M^{\emptyset, B_{+}} \oplus M^{\emptyset, \beta \uparrow B_{+}}\right) .
$$

Here we used the fact that $B_{+}$is the lowest alcove in the intersection of $\{D \in \mathscr{A} \mid$ $\left.D \succeq B_{-}\right\}$with the $\widehat{\mathcal{W}}^{\beta}$-orbit of $B_{+}$. 
After inducing from $\widehat{S}_{k}$ to $S_{k}$, the first image is $\Psi M\left(B_{-}, \beta\right)$ and the second is $\Psi M\left(B_{+}, \beta\right)$, and we deduce

$$
\begin{aligned}
\Psi^{s} \vartheta_{\text {on }} M(B, \beta) & =\Psi M\left(B_{-}, \beta\right) \oplus \Psi M\left(B_{+}, \beta\right) \\
& =\mathcal{T}_{\text {on }} \Psi M(B, \beta) .
\end{aligned}
$$

The case $\beta \uparrow B=B$ : This means that $\beta \uparrow B_{-}=B_{+}$. We want to determine

$$
\operatorname{im}\left(\left(\vartheta_{\text {on }} M\right)_{\succeq B}^{\beta} \rightarrow\left(\vartheta_{\text {on }} M\right)^{\emptyset, B}\right) \text {. }
$$

As before we have that $\left(\vartheta_{\text {on }} M\right)_{\succeq B}^{\beta}=M_{\succeq B_{-}}^{\beta}$ and $\left(\vartheta_{\text {on }} M\right)^{\emptyset, B}=M^{\emptyset, B_{-}} \oplus M^{\emptyset, B_{+}}$. So we have

$$
\operatorname{im}\left(\left(\vartheta_{\text {on }} M\right)_{\succeq B}^{\beta} \rightarrow\left(\vartheta_{\text {on }} M\right)^{\emptyset, B}\right)=\operatorname{im}\left(M_{\succeq B_{-}}^{\beta} \rightarrow M^{\emptyset, B_{-}} \oplus M^{\emptyset, B_{+}}\right) .
$$

Hence $\Psi^{s} \vartheta_{\text {on }} M(B, \beta)=\Psi M\left(B_{-}, \beta\right)=\mathcal{T}_{\text {on }} \Psi M(B, \beta)$.

This finishes part of the proof: By now we showed that the functors $\Psi^{s} \circ \vartheta_{\text {on }}$ and $\mathcal{T}_{\text {on }} \circ \Psi$ are isomorphic. Next we have to determine $\Psi \vartheta_{\text {out }} N(A, \beta)$.

The case $\beta \uparrow \bar{A} \neq \bar{A}$ : In this case the alcoves $A$ and $A s$ lie in different $\widehat{\mathcal{W}}^{\beta}$-orbits. Let us denote by $\Theta \in \widehat{\mathcal{W}}^{\beta} \backslash \mathscr{A}$ the orbit of $A$. We want to determine

$$
\begin{aligned}
& \operatorname{im}\left(\left(\vartheta_{\text {out }} N\right)_{\succeq A}^{\beta} \rightarrow\left(\vartheta_{\text {out }} N\right)^{\emptyset, A} \oplus\left(\vartheta_{\text {out }} N\right)^{\emptyset, \beta \uparrow A}\right) \\
= & \operatorname{im}\left(\left(\vartheta_{\text {out }} N\right)_{\succeq A}^{\beta, \Theta} \rightarrow\left(\vartheta_{\text {out }} N\right)^{\emptyset, A} \oplus\left(\vartheta_{\text {out }} N\right)^{\emptyset, \beta \uparrow A}\right) .
\end{aligned}
$$

By Lemma 3.5 we have $\left(\vartheta_{\text {out }} N\right)_{\succeq A}^{\beta, \Theta}=N_{\succeq \bar{A}}^{\beta, \bar{\Theta}}$, where $\bar{\Theta}$ is the image of $\Theta$ in $\widehat{\mathcal{W}}^{\beta} \backslash \mathscr{A}^{s}$. We already showed that $\left(\vartheta_{\text {out }} N\right)^{\emptyset, A}=N^{\emptyset, \bar{A}}$ and $\left(\vartheta_{\text {out }} N\right)^{\emptyset, \beta \uparrow A}=N^{\emptyset, \overline{\beta \uparrow A}}$. We have $\beta \uparrow \bar{A}=\overline{\beta \uparrow A}$ by Lemma 5.1, hence we can deduce

$$
\begin{aligned}
\operatorname{im}\left(\left(\vartheta_{\text {out }} N\right)_{\succeq A}^{\beta, \Theta} \rightarrow\left(\vartheta_{\text {out }} N\right)^{\emptyset, A} \oplus\left(\vartheta_{\text {out }} N\right)^{\emptyset, \beta \uparrow A}\right) & =\operatorname{im}\left(N_{\succeq \bar{A}}^{\beta, \bar{\Theta}} \rightarrow N^{\emptyset, \bar{A}} \oplus N^{\emptyset, \beta \uparrow \bar{A}}\right) \\
& =\operatorname{im}\left(N_{\succeq \bar{A}}^{\beta} \rightarrow N^{\emptyset, \bar{A}} \oplus N^{\emptyset, \beta \uparrow \bar{A}}\right),
\end{aligned}
$$

so $\Psi \vartheta_{\text {out }} N(A, \beta)=\Psi^{s} N(\bar{A}, \beta)=\mathcal{T}_{\text {out }} \Psi^{s} N(A, \beta)$.

The case $\beta \uparrow \bar{A}=\bar{A}$ and $A=\bar{A}_{-}$: In this case, $\bar{A}=\overline{\beta \uparrow A}=\beta \uparrow \bar{A}$. We want to determine

$$
\operatorname{im}\left(\left(\vartheta_{\text {out }} N\right)_{\succeq A}^{\beta} \rightarrow\left(\vartheta_{\text {out }} N\right)^{\emptyset, A} \oplus\left(\vartheta_{\text {out }} N\right)^{\emptyset, \beta \uparrow A}\right)
$$

The set $\{D \in \mathscr{A} \mid D \succeq A\}$ is $s$-invariant in the present case, so we can apply Lemma 3.5 and we get $\left(\vartheta_{\text {out }} N\right)_{\succeq A}^{\beta}=\vartheta_{\text {out }}\left(N_{\succeq \bar{A}}^{\beta}\right)$. By Lemma 3.2 we have $\vartheta_{\text {out }}\left(N^{\emptyset, \bar{A}}\right)=$ $\left(\vartheta_{\text {out }} N\right)^{\emptyset, A} \oplus\left(\vartheta_{\text {out }} N\right)^{\emptyset, \beta \uparrow A}$; hence

$$
\begin{aligned}
\operatorname{im}\left(\left(\vartheta_{\text {out }} N\right)_{\succeq A}^{\beta} \rightarrow\left(\vartheta_{\text {out }} N\right)^{\emptyset, A} \oplus\left(\vartheta_{\text {out }} N\right)^{\emptyset, \beta \uparrow A}\right) & =\operatorname{im}\left(\vartheta_{\text {out }}\left(N_{\succeq \bar{A}}^{\beta}\right) \rightarrow \vartheta_{\text {out }}\left(N^{\emptyset, \bar{A}}\right)\right) \\
& =\vartheta_{\text {out }}\left(\operatorname{im}\left(N_{\succeq \bar{A}}^{\beta} \rightarrow N^{\emptyset, \bar{A}}\right)\right)
\end{aligned}
$$

since $\vartheta_{\text {out }}$ is exact. Now

$$
\vartheta_{\text {out }}\left(\operatorname{im}\left(N_{\succeq \bar{A}}^{\beta} \rightarrow N^{\emptyset, \bar{A}}\right)\right)=\left\{\left(\beta_{n} x+y, y\right) \mid x, y \in \operatorname{im}\left(N_{\succeq \bar{A}}^{\beta} \rightarrow N^{\emptyset, \bar{A}}\right)\right\},
$$

where $n$ is such that $s_{\beta, n}(A)=\beta \uparrow A$. We deduce that

$$
\begin{aligned}
\Psi \vartheta_{\text {out }} N(A, \beta) & =\left\{(\beta x+y, y) \mid x, y \in \Psi^{s} N(\bar{A}, \beta)\right\} \\
& =\mathcal{T}_{\text {out }} \Psi^{s} N(A, \beta) .
\end{aligned}
$$


The case $\beta \uparrow \bar{A}=\bar{A}$ and $A=\bar{A}_{+}$: We want to determine

$$
\operatorname{im}\left(\overline{\left(\vartheta_{\text {out }} N\right)_{\succeq A}^{\beta}} \rightarrow \overline{\left(\vartheta_{\text {out }} N\right)^{\emptyset, A}} \oplus \overline{\left(\vartheta_{\text {out }} N\right)^{\emptyset, \beta \uparrow A}}\right) .
$$

By Lemma 3.2 we have a decomposition

$$
\left(\vartheta_{\text {out }} N\right)^{\beta}=\bigoplus_{\Theta \in \widehat{\mathcal{W}}^{\beta} \backslash \mathscr{A}}\left(\vartheta_{\text {out }} N\right)^{\beta, \Theta}
$$

Denote by $\Theta$ the $\widehat{\mathcal{W}}^{\beta}$-orbit of $A$. It also contains $\beta \uparrow A$, so

$$
\begin{aligned}
& \operatorname{im}\left(\left(\vartheta_{\text {out }} N\right)_{\succeq A}^{\beta} \rightarrow\left(\vartheta_{\text {out }} N\right)^{\emptyset, A} \oplus\left(\vartheta_{\text {out }} N\right)^{\emptyset, \beta \uparrow A}\right) \\
& =\operatorname{im}\left(\left(\vartheta_{\text {out }} N\right)_{\succeq A}^{\beta, \Theta} \rightarrow\left(\vartheta_{\text {out }} N\right)^{\emptyset, A} \oplus\left(\vartheta_{\text {out }} N\right)^{\emptyset, \beta \uparrow A}\right) .
\end{aligned}
$$

For notational convenience we set $N^{\prime}:=N^{\beta, \bar{\Theta}}$, where $\bar{\Theta}$ is the image of $\Theta$ inside $\widehat{\mathcal{W}}^{\beta} \backslash \mathscr{A}^{s}$. Then

$$
\left(\vartheta_{\text {out }} N\right)^{\beta, \Theta}=\vartheta_{\text {out }}\left(N^{\beta, \bar{\Theta}}\right)=\vartheta_{\text {out }} N^{\prime} .
$$

Hence we want to determine

$$
\operatorname{im}\left(\left(\vartheta_{\text {out }} N^{\prime}\right)_{\succeq A} \rightarrow\left(\vartheta_{\text {out }} N^{\prime}\right)^{\emptyset, A} \oplus\left(\vartheta_{\text {out }} N^{\prime}\right)^{\emptyset, \beta \uparrow A}\right) .
$$

Let us consider the inclusion

$$
\left(\vartheta_{\text {out }} N^{\prime}\right)_{\succeq A} /\left(\vartheta_{\text {out }} N^{\prime}\right)_{\succeq \beta \uparrow A} \subset\left(\vartheta_{\text {out }} N^{\prime}\right)_{\succeq \beta \downarrow A} /\left(\vartheta_{\text {out }} N^{\prime}\right)_{\succeq \beta \uparrow A} .
$$

The right-hand side is supported on $\{\beta \downarrow A, A\}$, and the left-hand side is its subspace of elements supported on $\{A\}$.

Since $A=\bar{A}_{+}$, the sets $\{\succeq \beta \uparrow A\}$ and $\{\succeq \beta \downarrow A\}$ are $s$-invariant. Hence, by Lemma 3.5

$$
\begin{aligned}
& \left(\vartheta_{\text {out }} N^{\prime}\right)_{\succeq \beta \downarrow A}=\vartheta_{\text {out }}\left(N_{\succeq \overline{\beta \downarrow A}}^{\prime}\right), \\
& \left(\vartheta_{\text {out }} N^{\prime}\right)_{\succeq \beta \uparrow A}=\vartheta_{\text {out }}\left(N_{\succeq \overline{\beta \uparrow A}}^{\prime}\right) .
\end{aligned}
$$

Using the exactness of the functor $\vartheta_{\text {out }}$ we identify the inclusion $(*)$ with

$$
\begin{aligned}
\left(\vartheta_{\text {out }} N^{\prime}\right)_{\succeq A} /\left(\vartheta_{\text {out }} N^{\prime}\right)_{\succeq \beta \uparrow A} & \subset \vartheta_{\text {out }}\left(N_{\succeq \overline{\beta \downarrow A}}^{\prime} / N_{\succeq \overline{\beta \uparrow A}}^{\prime}\right) \\
& =\widehat{\mathcal{Z}} \otimes_{\widehat{\mathcal{Z}}^{s}} N_{\succeq \overline{\beta \downarrow A}}^{\prime} / N_{\succeq \overline{\beta \uparrow A}}^{\prime} .
\end{aligned}
$$

Now $N_{\succeq \overline{\beta \downarrow A}}^{\prime} / N_{\succeq \overline{\beta \uparrow A}}^{\prime}$ is supported on $\{\bar{A}\}$; hence

$$
\begin{aligned}
\widehat{\mathcal{Z}} \otimes_{\widehat{\mathcal{Z}}^{s}} N_{\succeq \overline{\beta \downarrow A}}^{\prime} / N_{\succeq \overline{\beta \uparrow A}}^{\prime} & =\widehat{\mathcal{Z}}(\{A, \beta \downarrow A\}) \otimes_{\widehat{S}_{k}} N_{\succeq \overline{\beta \downarrow A}}^{\prime} / N_{\succeq \overline{\beta \uparrow A}}^{\prime} \\
& =\left\{\left(\beta_{n} x+y, y\right) \mid x, y \in N_{\succeq \overline{\beta \downarrow A}}^{\prime} / N_{\succeq \overline{\beta \uparrow A}}^{\prime}\right\} .
\end{aligned}
$$

Here $n$ is such that $s_{\beta, n}(A)=\beta \downarrow A$. Recall that the inclusion $(*)$ is the inclusion of the subspace of elements supported on $\{A\}$. We conclude

$$
\left(\vartheta_{\text {out }} N^{\prime}\right)_{\succeq A} /\left(\vartheta_{\text {out }} N^{\prime}\right)_{\succeq \beta \uparrow A}=\left(\beta_{n}, 0\right) \cdot\left(\vartheta_{\text {out }} N^{\prime}\right)_{\succeq \beta \downarrow A} /\left(\vartheta_{\text {out }} N^{\prime}\right)_{\succeq \beta \uparrow A} .
$$

Let $z=\left(z_{C}\right)_{C \in \mathscr{A}} \in \widehat{\mathcal{Z}}$ be an element with $z_{\beta \downarrow A}=0, z_{A} \equiv \beta \bmod \delta$ and $z_{\beta \uparrow A} \equiv 0$ $\bmod \delta$. Such an element exists by Lemma 2.2. From the last identity we deduce

$$
\overline{\left(\vartheta_{\text {out }} N^{\prime}\right)_{\succeq A}}=\overline{\left(\vartheta_{\text {out }} N^{\prime}\right)_{\succeq \beta \uparrow A}}+\overline{z \cdot\left(\vartheta_{\text {out }} N^{\prime}\right)_{\succeq \beta \downarrow A}} .
$$


We want to determine

$$
\operatorname{im}\left(\overline{\left(\vartheta_{\text {out }} N^{\prime}\right)_{\succeq A}} \rightarrow \overline{\left(\vartheta_{\text {out }} N^{\prime}\right)^{\emptyset, A}} \oplus \overline{\left(\vartheta_{\text {out }} N^{\prime}\right)^{\emptyset, \beta \uparrow A}}\right) .
$$

Now the image of $\overline{\left(\vartheta_{\text {out }} N^{\prime}\right)_{\succeq \beta \uparrow A}}$ inside $\overline{\left(\vartheta_{\text {out }} N^{\prime}\right)^{\emptyset, A}} \oplus \overline{\left(\vartheta_{\text {out }} N^{\prime}\right)^{\emptyset, \beta \uparrow A}}$ is contained in the second summand, while the image of $\overline{z \cdot\left(\vartheta_{\text {out }} N^{\prime}\right)_{\succeq \beta \downarrow A}}$ is contained in the first, since $z_{\beta \uparrow A} \equiv 0 \bmod \delta$.

We have

$$
\begin{aligned}
\operatorname{im}\left(\overline{\left(\vartheta_{\text {out }} N^{\prime}\right)_{\succeq \beta \uparrow A}} \rightarrow \overline{\left(\vartheta_{\text {out }} N^{\prime}\right)^{\emptyset, \beta \uparrow A}}\right) & =\operatorname{im}\left(\overline{N_{\succeq}^{\prime} \overline{\beta \uparrow A}} \rightarrow \overline{N^{\prime \emptyset, \overline{\beta \uparrow A}}}\right) \\
& =\operatorname{im}\left(\overline{N_{\succeq}^{\beta} \overline{\beta \uparrow A}} \rightarrow \overline{N^{\emptyset, \overline{\beta \uparrow A}}}\right) \\
& =\Psi^{s} N(\overline{\overline{\beta \uparrow A}}, \beta)
\end{aligned}
$$

and

$$
\begin{aligned}
\operatorname{im}\left(\overline{z \cdot\left(\vartheta_{\text {out }} N^{\prime}\right)_{\succeq \beta \downarrow A}} \rightarrow \overline{\left(\vartheta_{\text {out }} N^{\prime}\right)^{\emptyset, A}}\right) & =\beta \cdot \operatorname{im}\left(\overline{N_{\succeq \bar{A}}^{\prime}} \rightarrow \overline{N^{\prime \emptyset, \bar{A}}}\right) \\
& =\beta \cdot \operatorname{im}\left(\overline{N_{\succeq \bar{A}}^{\beta}} \rightarrow \overline{N^{\emptyset, \bar{A}}}\right) \\
& =\beta \cdot \Psi^{s} N(\bar{A}, \beta) .
\end{aligned}
$$

Hence we showed that

$$
\begin{aligned}
\Psi \vartheta_{\text {out }} N(A, \beta) & =\beta \cdot \Psi^{s} N(\bar{A}, \beta) \oplus \Psi^{s} N(\overline{\beta \uparrow A}, \beta) \\
& =\mathcal{T}_{\text {out }} \Psi^{s} N(A, \beta) .
\end{aligned}
$$

We have now considered all possible cases and hence have constructed an isomorphism $\Psi \circ \vartheta_{\text {out }} \cong \mathcal{T}_{\text {out }} \circ \Psi^{s}$ of functors. This also finishes the proof of our theorem.

5.6. The objects $M(A, \beta) \subset M(A) \oplus M(\beta \uparrow A)$. In this section we provide a result which is needed in the proof of Proposition 6.3 .

Fix $\beta \in R^{+}$. Choose $A \in \mathscr{F}$ and suppose that $\beta \uparrow A \neq A$. Let $\mathcal{K}_{k}^{\beta}(A)$ be the category whose objects are given by the data $(M, M(A), M(\beta \uparrow A))$, where $M(A)$ and $M(\beta \uparrow A)$ are $S_{k}^{\emptyset}$-modules and $M$ is an $S_{k}^{\beta}$-submodule of $M(A) \oplus M(\beta \uparrow A)$. Let the morphisms in $\mathcal{K}_{k}^{\beta}(A)$ be the obvious ones.

Let $V_{A}^{\beta} \in \mathcal{K}_{k}^{\beta}(A)$ be given by the inclusion $S_{k}^{\beta} \subset S_{k}^{\emptyset} \oplus 0$, let $V_{\beta \uparrow A}^{\beta}$ be given by the inclusion $S_{k}^{\beta} \subset 0 \oplus S_{k}^{\emptyset}$, and let $P_{A}^{\beta}$ be given by the inclusion $\{(\beta x+y, y) \mid x, y \in$ $\left.S_{k}^{\beta}\right\} \subset S_{k}^{\emptyset} \oplus S_{k}^{\emptyset}$.

If $\beta \uparrow A=A$ we define the category $\mathcal{K}_{k}^{\beta}(A)$ likewise, with objects given by the data $(M, M(A))$. We have a standard object $V_{A}^{\beta} \in \mathcal{K}_{k}^{\beta}(A)$ given by the inclusion $S_{k}^{\beta} \subset S_{k}^{\emptyset}$.

For $M \in \mathcal{M}_{k}, A \in \mathscr{A}$ and $\beta \in R^{+}$we can consider $M(A, \beta) \subset M(A) \oplus M(\beta \uparrow A)$ as an object in $\mathcal{K}_{k}^{\beta}(A)$. If $s \in \widehat{\mathcal{S}}$, the same holds for $M \in \mathcal{M}_{k}^{s}$ and $A \in \mathscr{A}^{s}$.

Lemma 5.6. Suppose $M \in \mathcal{M}_{k}$ and $A \in \mathscr{A}$. Then $M(A, \beta)$ is isomorphic to a direct sum of copies of $V_{A}^{\beta}, V_{\beta \uparrow A}^{\beta}$ and $P_{A}^{\beta}$. A similar statement holds for objects in $\mathcal{M}_{k}^{s}$. 
Proof. The statement is readily verified for $M=P_{0}$. If the lemma is true for $M$, then it is also true for each direct summand of $M$. Hence it is enough to show that if the lemma holds for $M$ and if $s \in \widehat{\mathcal{S}}$, then it also holds for $\mathcal{T}_{\text {on }}^{s}(M)\left(\mathcal{T}_{\text {out }}^{s}(M)\right.$, resp.). Now this is easily verified after a quick look at the definition of translation functors.

Lemma 5.7. Fix $M \in \mathcal{M}_{k}$ and $A \in \mathscr{A}$. Choose invertible elements $a, b \in S_{k}^{\beta}$ such that $a \equiv b \bmod \beta$. Then $(a, 1) M(A, \beta)=(b, 1) M(A, \beta)$. A similar statement holds for objects in $\mathcal{M}_{k}^{s}$.

Proof. By Lemma 5.6, $M(A, \beta)$ is isomorphic to a direct sum of copies of $V_{A}^{\beta}, V_{\beta \uparrow A}^{\beta}$ and $P_{A}^{\beta}$. For $V_{A}^{\beta}$ and $V_{\beta \uparrow A}^{\beta}$ the claim is clear. For the case of $P_{A}^{\beta}$ we observe that the inclusions

$$
\begin{aligned}
& (a, 1) \cdot\left\{(\beta x+y, y) \mid x, y \in S_{k}^{\beta}\right\} \subset S_{k}^{\emptyset} \oplus S_{k}^{\emptyset}, \\
& (b, 1) \cdot\left\{(\beta x+y, y) \mid x, y \in S_{k}^{\beta}\right\} \subset S_{k}^{\emptyset} \oplus S_{k}^{\emptyset}
\end{aligned}
$$

coincide, since the left-hand spaces are generated by $(a, 1)$ and $(\beta, 0)$, and by $(b, 1)$ and $(\beta, 0)$, resp., and since $a \equiv b \bmod \beta$.

\section{The translation functors of Andersen, Jantzen and Soergel}

The definition of the translation functors on $\mathcal{K}$ that is given by Andersen, Jantzen and Soergel in AJS94 is different from ours. In this section we will show that both versions lead to equivalent categories of special objects. For this we construct autoequivalences $\gamma: \mathcal{K}_{k} \rightarrow \mathcal{K}_{k}$ and $\gamma^{s}: \mathcal{K}_{k}^{s} \rightarrow \mathcal{K}_{k}^{s}$ for each $s \in \widehat{\mathcal{S}}$ that intertwine the sets of translation functors.

First we recall the definition of Andersen, Jantzen and Soergel. Let $R^{-}=-R^{+}$ be the system of negative roots and fix $s \in \widehat{\mathcal{S}}$. For $A \in \mathscr{A}$ and $\beta \in R^{+}$we define an element $a_{A}^{\beta} \in S_{k}^{\emptyset}$ by

$$
a_{A}^{\beta}:= \begin{cases}-\alpha_{\bar{A}} & \text { if } s_{\beta}\left(\alpha_{\bar{A}}\right) \in R^{-}, A=\bar{A}_{+}, \\ \alpha_{\bar{A}}^{-1} & \text { if } s_{\beta}\left(\alpha_{\bar{A}}\right) \in R^{-}, A=\bar{A}_{-}, \\ 1 & \text { if } s_{\beta}\left(\alpha_{\bar{A}}\right) \in R^{+} .\end{cases}
$$

(Recall that $\alpha_{\bar{A}}$ is the positive root associated to the hyperplane that contains $\bar{A}$.)

In [AJS94 the translation functors on the wall $\mathcal{T}_{\text {on }}^{\prime}=\mathcal{T}_{\text {on }}^{\prime s}: \mathcal{K}_{k} \rightarrow \mathcal{K}_{k}^{s}$ and out of the wall $\mathcal{T}_{\text {out }}^{\prime}=\mathcal{T}_{\text {out }}^{\prime s}: \mathcal{K}_{k}^{s} \rightarrow \mathcal{K}_{k}$ were defined as follows. For $M \in \mathcal{K}_{k}, B \in \mathscr{A}^{s}$ and $\beta \in R^{+}$set

$$
\begin{aligned}
\mathcal{T}_{\text {on }}^{\prime} M(B) & :=M\left(B_{-}\right) \oplus M\left(B_{+}\right), \\
\mathcal{T}_{\text {on }}^{\prime} M(B, \beta) & := \begin{cases}\left(\left(a_{B_{-}}^{\beta}\right)^{-1}, 1\right) M\left(B_{-}, \beta\right) & \text { if } \beta \uparrow B=B, \\
\left(\left(a_{B_{-}}^{\beta}\right)^{-1}, 1\right) M\left(B_{-}, \beta\right) & \\
\oplus\left(\left(a_{B_{+}}^{\beta}\right)^{-1}, 1\right) M\left(B_{+}, \beta\right) & \text { if } \beta \uparrow B \neq B .\end{cases}
\end{aligned}
$$


For $N \in \mathcal{K}_{k}^{s}, A \in \mathscr{A}$ and $\beta \in R^{+}$set

$$
\begin{aligned}
& \mathcal{T}_{\text {out }}^{\prime} N(A):= N(\bar{A}), \\
& \mathcal{T}_{\text {out }}^{\prime} N(A, \beta):= \begin{cases}\left\{\left(x+a_{A}^{\beta} y, y\right) \mid x, y \in N(\bar{A}, \beta)\right\} & \text { if } \beta \uparrow \bar{A}=\bar{A} \\
N(\bar{A}, \beta) \oplus N(\overline{\beta \uparrow A}, \beta) & \text { and } A=\bar{A}_{-}, \\
\left(a_{A}^{\beta}, 1\right) N(\bar{A}, \beta) & \text { if } \beta \uparrow \bar{A}=\bar{A} \\
\text { and } A=\bar{A}_{+},\end{cases}
\end{aligned}
$$

Set $\mathcal{T}^{\prime s}:=\mathcal{T}_{\text {out }}^{s} \circ \mathcal{T}_{\text {on }}^{s}$ and define $\mathcal{M}_{k}^{\prime} \subset \mathcal{K}_{k}$ in analogy to $\mathcal{M}_{k}$ (see Definition 5.3), i.e. by replacing the translation functors $\mathcal{T}^{s}$ by $\mathcal{T}^{\prime s}$. The main result of this section is the following.

Theorem 6.1. There is an equivalence $\gamma: \mathcal{M}_{k} \stackrel{\sim}{\rightarrow} \mathcal{M}_{k}^{\prime}$ that intertwines the translation functors on both sides; i.e., for all $s \in \widehat{\mathcal{S}}$ the following diagram commutes naturally:

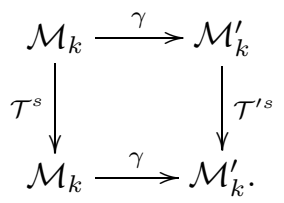

The proof of the theorem will occupy the remainder of this section.

6.1. Adding constants. Let $\mathscr{O} \subset \mathscr{F}$ be an orbit of facets, and choose for each $A \in \mathscr{O}$ and each $\beta \in R^{+}$invertible scalars $\gamma_{A}^{\beta,-}, \gamma_{A}^{\beta,+} \in S_{k}^{\emptyset}$ if $\beta \uparrow A \neq A$, and an invertible scalar $\gamma_{A}^{\beta} \in S_{k}^{\emptyset}$ if $\beta \uparrow A=A$. Denote by $\gamma_{\mathscr{O}}: \mathcal{K}_{k}(\mathscr{O}) \rightarrow \mathcal{K}_{k}(\mathscr{O})$ the following functor. For $A \in \mathscr{O}, \beta \in R^{+}$and $M \in \mathcal{K}_{k}(\mathscr{O})$ define

$$
\begin{aligned}
\gamma_{\mathscr{O}} M(A) & :=M(A), \\
\gamma_{\mathscr{O}} M(A, \beta) & := \begin{cases}\left(\gamma_{A}^{\beta,-}, \gamma_{A}^{\beta,+}\right) \cdot M(A, \beta) & \text { if } \beta \uparrow A \neq A, \\
\gamma_{A}^{\beta} \cdot M(A, \beta) & \text { if } \beta \uparrow A=A .\end{cases}
\end{aligned}
$$

If $f=\left(f_{A}\right)_{A \in \mathscr{O}}: M \rightarrow N$ is a morphism in $\mathcal{K}_{k}(\mathscr{O})$, then $f_{A} \oplus f_{\beta \uparrow A}\left(f_{A}\right.$, resp.) maps $M(A, \beta)$ into $N(A, \beta)$ if $\beta \uparrow A \neq A$ (if $\beta \uparrow A=A$, resp.) for each $A \in \mathscr{O}, \beta \in R^{+}$. Hence $f_{A} \oplus f_{\beta \uparrow A}\left(f_{A}\right.$, resp. $)$ also maps $\left(\gamma_{A}^{\beta,-}, \gamma_{A}^{\beta,+}\right) \cdot M(A, \beta)\left(\gamma_{A}^{\beta} \cdot M(A, \beta)\right.$, resp. $)$ into $\left(\gamma_{A}^{\beta,-}, \gamma_{A}^{\beta,+}\right) \cdot N(A, \beta)\left(\gamma_{A}^{\beta} \cdot M(A, \beta)\right.$, resp. $)$; hence the collection $\left(f_{A}\right)_{A \in \mathscr{O}}$ also yields a morphism $\gamma_{\mathscr{O}} f: \gamma_{\mathscr{O}} M \rightarrow \gamma_{\mathscr{O}} N$. So our choice of scalars indeed gives a functor $\gamma_{\mathscr{O}}: \mathcal{K}_{k}(\mathscr{O}) \rightarrow \mathcal{K}_{k}(\mathscr{O})$.

Proposition 6.2. The functor $\gamma_{\mathscr{O}}: \mathcal{K}_{k}(\mathscr{O}) \rightarrow \mathcal{K}_{k}(\mathscr{O})$ is an equivalence of categories.

Proof. The inverse functor $\gamma_{\mathscr{O}}^{-1}$ is given by the inverse scalars.

Now suppose we have chosen scalars as above for each alcove and each wall. This gives us functors $\gamma=\gamma_{\mathscr{A}}: \mathcal{K}_{k} \rightarrow \mathcal{K}_{k}$ and $\gamma^{s}=\gamma_{\mathscr{A}^{s}}: \mathcal{K}_{k}^{s} \rightarrow \mathcal{K}_{k}^{s}$ for each $s \in \widehat{\mathcal{S}}$. For each choice of scalars we obviously have $\gamma P_{0} \cong P_{0}$. Define

$$
\begin{array}{ccc}
\mathcal{T}_{\text {on }}^{\gamma}=\mathcal{T}_{\text {on }}^{\gamma s}:=\gamma^{s,-1} \circ \mathcal{T}_{\text {on }}^{\prime s} \circ \gamma: & \mathcal{K}_{k} \rightarrow \mathcal{K}_{k}^{s}, \\
\mathcal{T}_{\text {out }}^{\gamma}=\mathcal{T}_{\text {out }}^{\gamma s}:=\gamma^{-1} \circ \mathcal{T}_{\text {out }}^{s} \circ \gamma^{s}: & \mathcal{K}_{k}^{s} \rightarrow \mathcal{K}_{k} .
\end{array}
$$


Theorem 6.1 follows from the next statement.

Proposition 6.3. One can choose scalars as above for each alcove and each wall such that for all $s \in \widehat{\mathcal{S}}$ we have $\mathcal{T}_{\text {out }}^{\gamma s} \cong \mathcal{T}_{\text {out }}^{s}$ and $\mathcal{T}_{\text {on }}^{\gamma s} \cong \mathcal{T}_{\text {on }}^{s}$ when restricted to $\mathcal{M}_{k}$ and $\mathcal{M}_{k}^{s}$, respectively.

6.2. A choice of constants. For alcoves $A$ and $A^{\prime}$ with $A \preceq A^{\prime}$, define $H\left(A, A^{\prime}\right)$ as the set of all reflection hyperplanes that separate $A$ and $A^{\prime}$, i.e. as the set of all $H_{\alpha, n}$ with $A \subset H_{\alpha, n}^{-}$and $A^{\prime} \subset H_{\alpha, n}^{+}$. Let $\beta \in R^{+}$. We will now define a nonzero constant $d_{F}^{\beta} \in S_{k}^{\emptyset}$ for each facet $F$ that is an alcove or a wall. Suppose first that $F$ is an alcove $A \in \mathscr{A}$. Let $\alpha_{1}, \ldots, \alpha_{n} \in R^{+}$be the positive roots associated to the hyperplanes in $H(A, \beta \uparrow A)$, and set

$$
d_{A}^{\beta}:=\prod_{\substack{i=1, \ldots, n \\ s_{\beta}\left(\alpha_{i}\right) \in R^{-}}} \alpha_{i}^{-1} .
$$

For a wall $B \in \mathscr{W}$ set

$$
d_{B}^{\beta}:= \begin{cases}\alpha_{B} \cdot d_{B_{-}}^{\beta} & \text { if } s_{\beta}\left(\alpha_{B}\right) \in R^{-}, \\ d_{B_{-}}^{\beta} & \text { if } s_{\beta}\left(\alpha_{B}\right) \in R^{+} .\end{cases}
$$

Lemma 6.4. Let $A \in \mathscr{A}$ and $B \in \mathscr{W}$.

(1) $d_{A}^{\beta} \in \beta^{-1} S_{k}^{\beta}$, and $\beta \cdot d_{A}^{\beta}$ is invertible in $S_{k}^{\beta}$.

(2) If $B$ is of type $\beta$ (i.e. $\beta \uparrow B=B$ ), then $d_{B_{-}}^{\beta}=\beta^{-1}$ and $d_{B}^{\beta}=1$.

(3) If $B$ is not of type $\beta$ (i.e. $\beta \uparrow B \neq B$ ), then

$$
d_{B_{+}}^{\beta}= \begin{cases}\alpha_{B} \cdot\left(-s_{\beta}\left(\alpha_{B}\right)\right) \cdot d_{B_{-}}^{\beta} & \text { if } s_{\beta}\left(\alpha_{B}\right) \in R^{-}, \\ d_{B_{-}}^{\beta} & \text { if } s_{\beta}\left(\alpha_{B}\right) \in R^{+} .\end{cases}
$$

Proof. The alcoves $A$ and $\beta \uparrow A$ are separated by exactly one hyperplane of type $\beta$, and it follows that $d_{A}^{\beta} \in \beta^{-1} S_{k}^{\beta}$ and that $\beta \cdot d_{A}^{\beta}$ is invertible in $S_{k}^{\beta}$. Hence we have (1).

Only the hyperplane containing $B$ separates $B_{-}$and $B_{+}$, and if it is of type $\beta$, then $\beta \uparrow B_{-}=B_{+}$and we deduce that $d_{B_{-}}^{\beta}=\beta^{-1}$ and $d_{B}^{\beta}=1$ directly from the definitions. Hence we proved claim (2).

Suppose $B$ is not of type $\beta$. Then $\left\{\beta \uparrow B_{-}, \beta \uparrow B_{+}\right\}=\left\{(\beta \uparrow B)_{-},(\beta \uparrow B)_{+}\right\}$. Denote by $H_{1}$ the reflection hyperplane separating $B_{-}$and $B_{+}$and by $H_{2}$ the hyperplane separating $(\beta \uparrow B)_{-}$and $(\beta \uparrow B)_{+}$. For each reflection hyperplane $H \neq H_{1}$ we have $B_{-} \subset H^{-}$if and only if $B_{+} \subset H^{-}$, and the analogous result holds for $(\beta \uparrow B)_{-}$and $(\beta \uparrow B)_{+}$.

If $H_{1}=H_{2}$, then $B_{-}$and $\beta \uparrow B_{-}$lie on the same side of $H_{1}$ and we deduce that $H\left(B_{-}, \beta \uparrow B_{-}\right)=H\left(B_{+}, \beta \uparrow B_{+}\right)$, so $d_{B_{-}}^{\beta}=d_{B_{+}}^{\beta}$. Moreover, $s_{\beta}$ stabilizes $H_{1}$, so $s_{\beta}\left(\alpha_{B}\right)=\alpha_{B}$ (since $\alpha_{B} \neq \beta$ ), so $s_{\beta}\left(\alpha_{B}\right) \in R^{+}$; hence $d_{B_{+}}^{\beta}=d_{B_{-}}^{\beta}$ is what we claimed in (3).

If $H_{1} \neq H_{2}$, then $H\left(B_{-}, \beta \uparrow B_{-}\right)=H\left(B_{+}, \beta \uparrow B_{+}\right) \cup\left\{H_{1}, H_{2}\right\}$. By definition, $H_{1}$ is of type $\alpha_{B}$. Then the type of $H_{2}$ is the positive root in $\left\{ \pm s_{\beta}\left(\alpha_{B}\right)\right\}$. Suppose that $s_{\beta}\left(\alpha_{B}\right) \in R^{+}$. Then for the calculation of $d_{B_{-}}^{\beta}$ we do not have to consider $\alpha_{B}$, nor $s_{\beta}\left(\alpha_{B}\right)\left(\right.$ as $\left.s_{\beta}\left(s_{\beta}\left(\alpha_{B}\right)\right)=\alpha_{B} \in R^{+}\right)$. Hence $d_{B_{+}}^{\beta}=d_{B_{-}}^{\beta}$ in this case. But if $s_{\beta}\left(\alpha_{B}\right) \in R^{-}$, then, as $s_{\beta}\left(-s_{\beta}\left(\alpha_{B}\right)\right)=-\alpha_{B} \in R^{-}$, we have to consider both $\alpha_{B}$ and $-s_{\beta}\left(\alpha_{B}\right)$ for the calculation of $d_{B_{-}}^{\beta}$; hence $d_{B_{-}}^{\beta}=\alpha_{B}^{-1} \cdot\left(-s_{\beta}\left(\alpha_{B}\right)\right)^{-1} \cdot d_{B_{+}}^{\beta}$. 
Proof of Proposition 6.3. Let $\gamma: \mathcal{K}_{k} \rightarrow \mathcal{K}_{k}$ and $\gamma^{s}: \mathcal{K}_{k}^{s} \rightarrow \mathcal{K}_{k}^{s}$ for $s \in \widehat{\mathcal{S}}$ be the functors associated to the following constants. For $A \in \mathscr{A}, B \in \mathscr{W}$ and $\beta \in R^{+}$ define

$$
\begin{aligned}
& \gamma_{A}^{\beta,+}:=1, \quad \gamma_{A}^{\beta,-}:=d_{A}^{\beta}, \\
& \gamma_{B}^{\beta,+}:=1, \quad \gamma_{B}^{\beta,-}:=d_{B}^{\beta}, \quad \text { if } \beta \uparrow B \neq B \text {, } \\
& \gamma_{B}^{\beta}:=1, \quad \text { if } \beta \uparrow B=B .
\end{aligned}
$$

Now fix $s \in \widehat{\mathcal{S}}$ and choose $M \in \mathcal{M}_{k}, N \in \mathcal{M}_{k}^{s}, A \in \mathscr{A}$ and $B \in \mathscr{A}^{s}$. Then

$$
\begin{aligned}
& \mathcal{T}_{\text {on }}^{\gamma} M(B)=M\left(B_{-}\right) \oplus M\left(B_{+}\right)=\mathcal{T}_{\text {on }} M(B), \\
& \mathcal{T}_{\text {out }}^{\gamma} N(A)=N(\bar{A})=\mathcal{T}_{\text {out }} N(A) .
\end{aligned}
$$

In order to prove the proposition it is enough to show that the subspaces associated to $\beta \in R^{+}$coincide.

The case $\beta \uparrow B=B$. Then $\alpha_{B}=\beta, a_{B_{-}}^{\beta}=\beta^{-1}, \gamma_{B_{-}}^{\beta,-}=\beta^{-1}, \gamma_{B_{-}}^{\beta,+}=\gamma_{B}^{\beta}=1$. Hence

$$
\begin{aligned}
\mathcal{T}_{\text {on }}^{\gamma} M(B, \beta) & =\left(\frac{\gamma_{B_{-}}^{\beta,-}}{a_{B_{-}}^{\beta} \cdot \gamma_{B}^{\beta}}, \frac{\gamma_{B_{-}}^{\beta,+}}{\gamma_{B}^{\beta}}\right) M\left(B_{-}, \beta\right) \\
& =M\left(B_{-}, \beta\right)=\mathcal{T}_{\text {on }} M(B, \beta) .
\end{aligned}
$$

The case $\beta \uparrow B \neq B$ and $s_{\beta}\left(\alpha_{B}\right) \in R^{-}$. Then $a_{B_{-}}^{\beta}=\alpha_{B}^{-1}, a_{B_{+}}^{\beta}=-\alpha_{B}$, $\gamma_{B_{-}}^{\beta,-}=d_{B_{-}}^{\beta}, \gamma_{B_{+}}^{\beta,-}=d_{B_{+}}^{\beta}, \gamma_{B}^{\beta,-}=d_{B}^{\beta}=\alpha_{B} \cdot d_{B_{-}}^{\beta}$ and $\gamma_{B_{-}}^{\beta,+}=\gamma_{B_{+}}^{\beta,+}=\gamma_{B}^{\beta,+}=1$. Moreover, $d_{B_{+}}^{\beta}=\alpha_{B} \cdot\left(-s_{\beta}\left(\alpha_{B}\right)\right) \cdot d_{B_{-}}^{\beta}$ by Lemma 6.4 Hence

$$
\begin{aligned}
\mathcal{T}_{\text {on }}^{\gamma} M(B, \beta) & =\left(\frac{\gamma_{B_{-}}^{\beta,-}}{a_{B_{-}}^{\beta} \gamma_{B}^{\beta,-}}, \frac{\gamma_{B_{-}}^{\beta,+}}{\gamma_{B}^{\beta,+}}\right) M\left(B_{-}, \beta\right) \oplus\left(\frac{\gamma_{B_{+}}^{\beta,-}}{a_{B_{+}}^{\beta} \gamma_{B}^{\beta,-}}, \frac{\gamma_{B_{+}}^{\beta,+}}{\gamma_{B}^{\beta,+}}\right) M\left(B_{+}, \beta\right) \\
& =\left(\frac{d_{B_{-}}^{\beta}}{\alpha_{B}^{-1} \alpha_{B} d_{B_{-}}^{\beta}}, 1\right) M\left(B_{-}, \beta\right) \oplus\left(\frac{d_{B_{+}}^{\beta}}{-\alpha_{B} \alpha_{B} d_{B_{-}}^{\beta}}, 1\right) M\left(B_{+}, \beta\right) \\
& =M\left(B_{-}, \beta\right) \oplus\left(\frac{s_{\beta}\left(\alpha_{B}\right)}{\alpha_{B}}, 1\right) M\left(B_{+}, \beta\right) .
\end{aligned}
$$

Now $s_{\beta}\left(\alpha_{B}\right) \equiv \alpha_{B} \bmod \beta$; hence $s_{\beta}\left(\alpha_{B}\right) \cdot \alpha_{B}^{-1} \equiv 1 \bmod \beta$. By Lemma 5.7, $\left(\frac{s_{\beta}\left(\alpha_{B}\right)}{\alpha_{B}}, 1\right) M\left(B_{+}, \beta\right)=M\left(B_{+}, \beta\right)$; hence $\mathcal{T}_{\text {on }}^{\gamma} M(B, \beta)=\mathcal{T}_{\text {on }} M(B, \beta)$.

The case $\beta \uparrow B \neq B$ and $s_{\beta}\left(\alpha_{B}\right) \in R^{+}$. Then $a_{B_{-}}^{\beta}=a_{B_{+}}^{\beta}=1, \gamma_{B_{-}}^{\beta,-}=d_{B_{-}}^{\beta}$, $\gamma_{B_{+}}^{\beta,-}=d_{B_{+}}^{\beta}, \gamma_{B}^{\beta,-}=d_{B}^{\beta}=d_{B_{-}}^{\beta}$ and $\gamma_{B_{-}}^{\beta,+}=\gamma_{B_{+}}^{\beta,+}=\gamma_{B}^{\beta,+}=1$. Moreover, $d_{B_{+}}^{\beta}=d_{B_{-}}^{\beta}$ by Lemma 6.4. Hence

$$
\begin{aligned}
\mathcal{T}_{\text {on }}^{\gamma} M(B, \beta) & =\left(\frac{\gamma_{B_{-}}^{\beta,-}}{a_{B_{-}}^{\beta} \gamma_{B}^{\beta,-}}, \frac{\gamma_{B_{-}}^{\beta,+}}{\gamma_{B}^{\beta,+}}\right) M\left(B_{-}, \beta\right) \oplus\left(\frac{\gamma_{B_{+}}^{\beta,-}}{a_{B_{+}}^{\beta} \gamma_{B}^{\beta,-}}, \frac{\gamma_{B_{+}}^{\beta,+}}{\gamma_{B}^{\beta,+}}\right) M\left(B_{+}, \beta\right) \\
& =\left(\frac{d_{B_{-}}^{\beta}}{d_{B_{-}}^{\beta}}, 1\right) M\left(B_{-}, \beta\right) \oplus\left(\frac{d_{B_{+}}^{\beta}}{d_{B_{-}}^{\beta}}, 1\right) M\left(B_{+}, \beta\right) \\
& =M\left(B_{-}, \beta\right) \oplus M\left(B_{+}, \beta\right)=\mathcal{T}_{\text {on }} M(B, \beta) .
\end{aligned}
$$

Hence we proved the proposition for $\mathcal{T}_{\text {on }}$. 
The case $\beta \uparrow \bar{A}=\bar{A}$ and $A=\bar{A}_{-}$. Then $\alpha_{\bar{A}}=\beta, a_{A}^{\beta}=\beta^{-1}, \gamma_{A}^{\beta,-}=\beta^{-1}$, $\gamma_{A}^{\beta,+}=1$ and $\gamma_{\frac{\beta}{A}}^{\beta}=1$ by Lemma 6.4. Hence

$$
\begin{aligned}
\mathcal{T}_{\text {out }}^{\gamma} N(A, \beta) & =\left\{\left(\frac{x+a_{A}^{\beta} y}{\gamma_{A}^{\beta,-}}, \frac{y}{\gamma_{A}^{\beta,+}}\right) \mid x, y \in \gamma_{\bar{A}}^{\beta} \cdot N(\bar{A}, \beta)\right\} \\
& =\{(\beta x+y, y) \mid x, y \in N(\bar{A}, \beta)\} \\
& =\mathcal{T}_{\text {out }} N(A, \beta) .
\end{aligned}
$$

The case $\beta \uparrow \bar{A}=\bar{A}$ and $A=\bar{A}_{+}$. Then $\gamma_{A}^{\beta,-}=d_{A}^{\beta}, \gamma_{A}^{\beta,+}=1$ and $\gamma_{\frac{\beta}{A}}^{\beta}=\gamma_{\frac{\beta}{\beta \uparrow A}}=1$ by Lemma 6.4. Hence

$$
\begin{aligned}
\mathcal{T}_{\text {out }}^{\gamma} N(A, \beta) & =\frac{\gamma_{\bar{A}}^{\beta}}{\gamma_{A}^{\beta,-}} N(\bar{A}, \beta) \oplus \frac{\gamma_{\overline{\beta \uparrow A}}^{\beta}}{\gamma_{A}^{\beta,+}} N(\overline{\beta \uparrow A}, \beta) \\
& =\left(d_{A}^{\beta}\right)^{-1} \cdot N(\bar{A}, \beta) \oplus N(\overline{\beta \uparrow A}, \beta) .
\end{aligned}
$$

Now $\beta \cdot d_{A}^{\beta}$ is invertible in $S_{k}^{\beta}$ by Lemma 6.4 hence $\left(d_{A}^{\beta}\right)^{-1} \cdot N(\bar{A}, \beta)=\beta \cdot N(\bar{A}, \beta)$, so $\mathcal{T}_{\text {out }}^{\gamma} N(A, \beta)=\mathcal{T}_{\text {out }} N(A, \beta)$.

The case $\beta \uparrow \bar{A} \neq \bar{A}$ and $s_{\beta}\left(\alpha_{\bar{A}}\right) \in R^{+}$. Then $a_{A}^{\beta}=1, \gamma_{A}^{\beta,-}=d_{A}^{\beta}, \gamma_{\bar{A}}^{\beta,-}=d_{\bar{A}}^{\beta}=$ $d_{\bar{A}_{-}}^{\beta}$ and $\gamma_{A}^{\beta,+}=\gamma_{\bar{A}}^{\beta,+}=1$. Moreover, $d_{\bar{A}_{+}}^{\beta}=d_{\bar{A}_{-}}^{\beta}$; hence in either case $\left(A=\bar{A}_{+}{ }^{\prime}\right.$ or $\left.A=\bar{A}_{-}\right)$we have $d_{A}^{\beta}=d_{\bar{A}_{-}}^{\beta}$. Then

$$
\begin{aligned}
\mathcal{T}_{\text {out }}^{\gamma} N(A, \beta) & =\left(\frac{a_{A}^{\beta} \cdot \gamma_{\bar{A}}^{\beta,-}}{\gamma_{A}^{\beta,-}}, \frac{\gamma_{\bar{A}}^{\beta,+}}{\gamma_{A}^{\beta,+}}\right) N(\bar{A}, \beta) \\
& =\left(\frac{d_{\bar{A}_{-}}^{\beta}, 1}{d_{A}^{\beta}}\right) N(\bar{A}, \beta) \\
& =\mathcal{T}_{\text {out }} N(A, \beta) .
\end{aligned}
$$

The case $\beta \uparrow \bar{A} \neq \bar{A}$ and $s_{\beta}\left(\alpha_{\bar{A}}\right) \in R^{-}$. Then $a_{\bar{A}_{-}}^{\beta}=\alpha_{\bar{A}^{-1}}^{-1} a_{\bar{A}_{+}}^{\beta}=-\alpha_{\bar{A}}$, $\gamma_{A}^{\beta,-}=d_{A}^{\beta}, \gamma_{\bar{A}}^{\beta,-}=d_{\bar{A}}^{\beta}=\alpha_{\bar{A}} \cdot d_{\bar{A}_{-}}^{\beta}$ and $\gamma_{A}^{\beta,+}=\gamma_{\bar{A}}^{\beta,+}=1$. Moreover, $d_{\bar{A}_{+}}^{\beta}=$ $\alpha_{\bar{A}} \cdot\left(-s_{\beta}\left(\alpha_{\bar{A}}\right)\right) \cdot d_{\bar{A}_{-}}^{\beta}$ by Lemma 6.4. If $A=\bar{A}_{-}$, then

$$
\begin{aligned}
\mathcal{T}_{\text {out }}^{\gamma} N(A, \beta) & =\left(\frac{a_{A}^{\beta} \cdot \gamma_{\bar{A}}^{\beta,-}}{\gamma_{A}^{\beta,-}}, \frac{\gamma_{\bar{A}}^{\beta,+}}{\gamma_{A}^{\beta,+}}\right) N(\bar{A}, \beta) \\
& =\left(\frac{\alpha_{\bar{A}}^{-1} \cdot \alpha_{\bar{A}} \cdot d_{\bar{A}_{-}}^{\beta}}{d_{A}^{\beta}}, 1\right) N(\bar{A}, \beta) \\
& =N(\bar{A}, \beta)=\mathcal{T}_{\text {out }} N(A, \beta) .
\end{aligned}
$$


If $A=\bar{A}_{+}$, then

$$
\begin{aligned}
\mathcal{T}_{\text {out }}^{\gamma} N(A, \beta) & =\left(\frac{a_{A}^{\beta} \cdot \gamma_{\bar{A}}^{\beta,-}}{\gamma_{A}^{\beta,-}}, \frac{\gamma_{\bar{A}}^{\beta,+}}{\gamma_{A}^{\beta,+}}\right) N(\bar{A}, \beta) \\
& =\left(\frac{-\alpha_{\bar{A}} \cdot \alpha_{\bar{A}} \cdot d_{\bar{A}_{-}}^{\beta}}{d_{\bar{A}_{+}}^{\beta}}, 1\right) N(\bar{A}, \beta) \\
& =\left(\frac{\alpha_{\bar{A}}}{s_{\beta}\left(\alpha_{\bar{A}}\right)}, 1\right) N(\bar{A}, \beta) .
\end{aligned}
$$

Now $s_{\beta}\left(\alpha_{\bar{A}}\right) \equiv \alpha_{\bar{A}} \bmod \beta$; hence $s_{\beta}\left(\alpha_{\bar{A}}\right)^{-1} \cdot \alpha_{\bar{A}} \equiv 1 \bmod \beta$. By Lemma 5.7 , $\left(s_{\beta}\left(\alpha_{\bar{A}}\right)^{-1} \cdot \alpha_{\bar{A}}, 1\right) N(\bar{A}, \beta)=N(\bar{A}, \beta) ;$ hence $\mathcal{T}_{\text {out }}^{\gamma} N(A, \beta)=\mathcal{T}_{\text {out }} N(A, \beta)$.

\section{Sheaves on the AfFine Flag VARIETy}

In this section we introduce the affine flag variety $\widehat{\mathcal{F} 1}$ associated to the connected, simply connected complex algebraic group with root datum $R$. It carries an action of an extended torus $\widehat{T}$. We define for a field $k$ the category $\mathcal{I}_{k}$ of "special $\widehat{T}$ equivariant sheaves" of $k$-vector spaces on $\widehat{\mathcal{F}}$. (In the following we call a "sheaf" any element in the derived category of sheaves on a topological space.) To each $w \in \widehat{\mathcal{W}}$ one associates a Schubert cell $\mathcal{O}_{w} \subset \widehat{\mathcal{F} l}$ and its closure $\overline{\mathcal{O}_{w}} \subset \widehat{\mathcal{F} 1}$, the corresponding Schubert variety. Note that each Schubert variety is a finite-dimensional complex projective variety. We show that each special sheaf is supported inside a Schubert variety.

For the applications to modular representation theory, only a subcategory $\mathcal{I}_{k}^{\circ}$ of $\mathcal{I}_{k}$ with finitely many indecomposable isomorphism classes (up to shifts) plays a role. We use the decomposition theorem to show that, if $k$ is a field with zero or big enough characteristic, the indecomposable objects in $\mathcal{I}_{k}^{\circ}$ are, up to a shift, the equivariant intersection cohomology sheaves $\mathrm{IC}_{\widehat{T}, y}$ on the Schubert varieties $\overline{\mathcal{O}_{y}}$, where $y$ comes from a finite subset $\widehat{\mathcal{W}}^{\circ}$ of $\widehat{\mathcal{W}}$. By a classical theorem of Kazhdan and Lusztig, the "characters" of these sheaves are known.

Then we consider the equivariant hypercohomology functor $\mathbb{H}_{\widehat{T}}^{*}$ and show that it can be considered as a functor from $\mathcal{I}_{k}$ to $\mathcal{H}_{k}$ and that the characters of sheaves yield characters of objects in $\mathcal{H}_{k}$.

In the following section we state some theorems about the structure of affine flag varieties. For details we refer to Kumar's book Kum02.

7.1. The affine flag variety. Let $G$ be a connected simply connected complex algebraic group. Let $T \subset B \subset G$ be a maximal torus and a Borel subgroup and suppose that $R$ is the root system of $G$ and that $R^{+} \subset R$ is the set of roots of $B$.

Denote by $G((t)):=\{f: \operatorname{Spec} \mathbb{C}((t)) \rightarrow G\}$ the associated loop group, and by $G[[t]]:=\{f:$ Spec $\mathbb{C}[[t]] \rightarrow G\} \subset G((t))$ the subgroup of loops that extend to zero. Let $I \subset G[[t]]$ be the Iwahori subgroup, i.e. the preimage of $B$ under the evaluation map $G[[t]] \rightarrow G, t \mapsto 0$. The set-theoretic quotient $\widehat{\mathcal{F} 1}=G((t)) / I$ carries a natural structure of an ind-variety and is called the affine flag variety.

To a simple affine reflection $s \in \widehat{\mathcal{S}}$ corresponds a minimal parabolic subgroup $P_{s} \subset G((t))$ that contains $I$. The set-theoretic quotient $\widehat{\mathcal{F}}_{s}:=G((t)) / P_{s}$ carries 
again an ind-variety structure and is called a partial affine flag variety. The natural $\operatorname{map} \pi_{s}: \widehat{\mathcal{F} l} \rightarrow \widehat{\mathcal{F}}_{s}$ is an ind-proper map of ind-varieties.

The set of $I$-orbits in $\widehat{\mathcal{F} l}$ can canonically be identified with the affine Weyl group $\widehat{\mathcal{W}}$. Denote the orbit corresponding to $w \in \widehat{\mathcal{W}}$ by $\mathcal{O}_{w} \subset \widehat{\mathcal{F}}$. Its Zariski closure $\overline{\mathcal{O}_{w}}$ is a finite-dimensional, projective variety and is called a Schubert variety. It is the union of the orbits $\mathcal{O}_{y}$ for all $y \leq w$. The set of $I$-orbits in $\widehat{\mathcal{F l}}_{s}$ can analogously be identified with $\widehat{\mathcal{W}}^{s}$, and for each $\bar{w} \in \widehat{\mathcal{W}}^{s}$ we denote the corresponding orbit by $\mathcal{O}_{\bar{w}}$. Its closure $\overline{\mathcal{O}_{\bar{w}}}$ is called a partial Schubert variety. We have $\pi_{s}\left(\mathcal{O}_{w}\right)=\mathcal{O}_{\bar{w}}$ and $\pi_{s}^{-1}\left(\mathcal{O}_{\bar{w}}\right)=\mathcal{O}_{w} \cup \mathcal{O}_{w s}$ for all $w \in \widehat{\mathcal{W}}$.

Let $\widehat{T}=T \times \mathbb{C}^{\times}$be the extended torus, and define a $\widehat{T}$-action on $G((t))$ by letting the first factor act by left multiplication and the second by rotating the loops. Then $\widehat{T}$ also acts on $\widehat{\mathcal{F} l}$ and on $\widehat{\mathcal{F l}}_{s}$ for all $s \in \widehat{\mathcal{S}}$. Each $\mathcal{O}_{w}$ contains a unique $\widehat{T}$-fixed point $p t_{w} \in \mathcal{O}_{w}$. Denote by $i_{w}:\left\{p t_{w}\right\} \rightarrow \widehat{\mathcal{F}}$ its inclusion.

7.2. The equivariant cohomology of Schubert varieties. Let $w \in \widehat{\mathcal{W}}$, and denote by $i: \overline{\mathcal{O}}_{w}{ }^{\widehat{T}} \rightarrow \overline{\mathcal{O}_{w}}$ the inclusion of the set of $\widehat{T}$-fixed points. Then $\overline{\mathcal{O}}_{w}{ }^{\widehat{T}}$ can be identified with the set $\{\leq w\}:=\{x \in \widehat{\mathcal{W}} \mid x \leq w\}$. In particular, it is finite. Now identify the character lattice $\operatorname{Hom}\left(\widehat{T}, \mathbb{C}^{\times}\right)$of the extended torus with the extended character lattice $\widehat{X}=X \oplus \mathbb{Z}$. Then $H_{\widehat{T}}^{*}(p t, \mathbb{Z})=S(\widehat{X})=\widehat{S}_{\mathbb{Z}}$. Consider the "localization map" on equivariant topology,

$$
i^{*}: H_{\widehat{T}}^{*}\left(\overline{\mathcal{O}_{w}}, \mathbb{Z}\right) \rightarrow H_{\widehat{T}}^{*}\left(\overline{\mathcal{O}_{w}} \widehat{T}^{\prime}, \mathbb{Z}\right)=\bigoplus_{x \leq w} \widehat{S}_{\mathbb{Z}}
$$

The following statement can be deduced from the results in Chapter XI of Kum02].

Theorem 7.1. Let $w \in \widehat{\mathcal{W}}$.

(1) The localization map is injective and induces an isomorphism

$$
H_{\widehat{T}}^{*}\left(\overline{\mathcal{O}_{w}}, \mathbb{Z}\right)=\left\{\left(z_{x}\right) \in \bigoplus_{x \leq w} \widehat{S}_{\mathbb{Z}} \mid \begin{array}{c}
z_{x} \equiv z_{t x} \bmod \alpha_{t} \\
\text { for all } t \in \widehat{\mathcal{T}}, x \leq w \text { with } t x \leq w
\end{array}\right\} .
$$

(2) Let $s \in \widehat{\mathcal{S}}$ and suppose $w s<w$. The pull-back map $\pi_{s}^{*}$ on equivariant cohomology induces an identification

$$
H_{\widehat{T}}^{*}\left(\overline{\mathcal{O}_{\bar{w}}}, \mathbb{Z}\right)=H_{\widehat{T}}^{*}\left(\overline{\mathcal{O}_{w}}, \mathbb{Z}\right)^{s} .
$$

(In part (2) we denote by $H_{\widehat{T}}^{*}\left(\overline{\mathcal{O}_{w}}, \mathbb{Z}\right)^{s} \subset H_{\widehat{T}}^{*}\left(\overline{\mathcal{O}_{w}}, \mathbb{Z}\right)$ the subspace of $\sigma_{s}$-invariant elements, i.e. the set of $\left(z_{x}\right)$ with $z_{x}=z_{x s}$ for all $x \leq w$.)

Now fix a field $k$ and let $\widehat{\mathcal{Z}}_{k}$ be the structure algebra over the field $k$ introduced in Section 2.4 Let $\widehat{\mathcal{Z}}_{k}(\{\leq w\})$ be its finite version corresponding to the set of vertices $\{x \in \widehat{\mathcal{W}} \mid x \leq w\}$. A quick look at the definition of the structure algebra gives the following result:

Corollary 7.2. For each field $k$ we have natural identifications $H_{\widehat{T}}^{*}\left(\overline{\mathcal{O}_{w}}, k\right)=$ $\widehat{\mathcal{Z}}_{k}(\{\leq w\})$ for all $w \in \widehat{\mathcal{W}}$ and $H_{\widehat{T}}^{*}\left(\overline{\mathcal{O}_{\bar{w}}}, k\right)=\widehat{\mathcal{Z}}_{k}(\{\leq w\})^{s}$ for all $w \in \widehat{\mathcal{W}}$ with $w s<w$. 
7.3. Special sheaves on $\widehat{\mathcal{F l}}$. Again let $k$ be a field of characteristic $\neq 2$. For a topological space $Y$ which is acted upon continuously by $\widehat{T}$ we denote by $\mathrm{D}_{\widehat{T}}(Y, k)$ the $\widehat{T}$-equivariant derived category of sheaves of $k$-modules with cohomology bounded from below. If $f: Y \rightarrow Y^{\prime}$ is a continuous map between such spaces that commutes with the $\widehat{T}$-actions, we denote by $f^{*}: \mathrm{D}_{\widehat{T}}\left(Y^{\prime}, k\right) \rightarrow \mathrm{D}_{\widehat{T}}(Y, k)$ and by $f_{*}: \mathrm{D}_{\widehat{T}}(Y, k) \rightarrow \mathrm{D}_{\widehat{T}}\left(Y^{\prime}, k\right)$ the equivariant pull-back and push-forward functors.

Denote by $[n]: \mathrm{D}_{\widehat{T}}(\widehat{\mathcal{F} l}, k) \rightarrow \mathrm{D}_{\widehat{T}}(\widehat{\mathcal{F} l}, k)$ the shift functor for $n \in \mathbb{Z}$. Note that the support of a sheaf $F \in \mathrm{D}_{\widehat{T}}(\widehat{\mathcal{F} l}, k)$, denoted by $\operatorname{supp} F$, is defined as the support of the underlying ordinary sheaf in $\mathrm{D}(\widehat{\mathcal{F}}, k)$, i.e. the union of the supports of the cohomology sheaves of the latter.

Let $k_{e} \in \mathrm{D}_{\widehat{T}}\left(\mathcal{O}_{e}, k\right)$ be the constant equivariant sheaf of rank one on the point orbit $\mathcal{O}_{e}$, and set $F_{e}:=i_{e *} k_{e} \in \mathrm{D}_{\widehat{T}}(\widehat{\mathcal{F}} 1, k)$.

Definition 7.3. The category of special (equivariant) sheaves is the full subcategory $\mathcal{I}_{k}$ of $\mathrm{D}_{\widehat{T}}(\widehat{\mathcal{F} l}, k)$ that consists of all objects that are isomorphic to a direct summand of a direct sum of sheaves of the form $\pi_{s}^{*} \pi_{s *} \cdots \pi_{t}^{*} \pi_{t *} F_{e}[n]$ for arbitrary sequences $s, \ldots, t \in \widehat{\mathcal{S}}$ and $n \in \mathbb{Z}$.

In $\mathrm{FW}$ we give a more intrinsic definition of this category as the category of parity sheaves on the affine flag manifold.

Lemma 7.4. The support of each object of $\mathcal{I}_{k}$ is contained in a Schubert variety; i.e., for each $F \in \mathcal{I}_{k}$ there exists $w \in \widehat{\mathcal{W}}$ with $\operatorname{supp} F \subset \overline{\mathcal{O}_{w}}$.

Proof. This is certainly true for $F_{e}$. If it is true for $F$, then it is also true for each isomorphic object, for $\pi_{s}^{*} \pi_{s *} F$, for $F[n]$ and for each direct summand of $F$. If it is true for $F$ and $G$, then it is also true for $F \oplus G$. The lemma follows.

7.4. The hypercohomology of special sheaves. Let $F \in \mathcal{I}_{k}$ and suppose that the support of $F$ is contained in $\overline{\mathcal{O}_{w}}$. Then the hypercohomology $\mathbb{H}_{\widehat{T}}^{*}(F)$ is naturally a module over the equivariant cohomology $H_{\widehat{T}}^{*}\left(\overline{\mathcal{O}_{w}}, k\right)$. The natural map $\widehat{\mathcal{Z}}_{k} \rightarrow$ $\widehat{\mathcal{Z}}_{k}(\{\leq w\}) \cong H_{\widehat{T}}^{*}\left(\overline{\mathcal{O}_{w}}, k\right)$ hence induces a $\widehat{\mathcal{Z}}_{k}$-module structure on $\mathbb{H}_{\widehat{T}}^{*}(F)$, so we can consider $\mathbb{H}_{\widehat{T}}^{*}$ as a functor from $\mathcal{I}_{k}$ to $\widehat{\mathcal{Z}}_{k}$-mod.

Proposition 7.5. Choose $F \in \mathcal{I}_{k}$ and $s \in \widehat{\mathcal{S}}$. Then there is a natural isomorphism

$$
\widehat{\mathcal{Z}}_{k} \otimes_{\widehat{\mathcal{Z}}_{k}^{s}} \mathbb{H}_{\widehat{T}}^{*}(F) \cong \mathbb{H}_{\widehat{T}}^{*}\left(\pi_{s}^{*} \pi_{s *} F\right)
$$

of $\widehat{\mathcal{Z}}_{k}$-modules.

Proof. Suppose that the support of $F$ is contained in $\overline{\mathcal{O}_{w}}$ for $w \in \widehat{\mathcal{W}}$. Without loss of generality we can assume that $w s<w$. Then $\pi_{s}^{*} \pi_{s *} F$ is supported on $\overline{\mathcal{O}_{w}}$ as well. As in [Soe00] one shows that we have an isomorphism

$$
H_{\widehat{T}}^{*}\left(\overline{\mathcal{O}_{w}}\right) \otimes_{H_{\widehat{T}}^{*}\left(\overline{\mathcal{O}_{\bar{w}}}\right)} \mathbb{H}_{\widehat{T}}^{*}(F)=\mathbb{H}_{\widehat{T}}^{*}\left(\pi_{s}^{*} \pi_{s *} F\right)
$$

of $H_{\widehat{T}}^{*}\left(\overline{\mathcal{O}_{w}}\right)$-modules. Now the statement follows from Corollary 7.2

Theorem 7.6. Equivariant hypercohomology gives a functor $\mathbb{H}_{\widehat{T}}^{*}: \mathcal{I}_{k} \rightarrow \mathcal{H}_{k}$ such that the following diagram commutes naturally for all $s \in \widehat{\mathcal{S}}$ : 


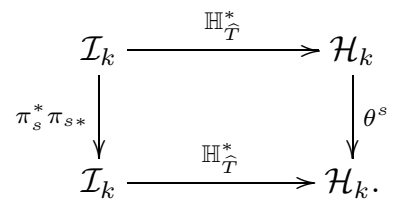

Moreover, we have $\operatorname{rk} \mathbb{H}_{\widehat{T}}^{*}\left(i_{x}^{*} F\right)=\operatorname{rk} \mathbb{H}_{\widehat{T}}^{*}(F)^{\emptyset, x}$ for each $F \in \mathcal{I}_{k}$ and $x \in \widehat{\mathcal{W}}$. (By $\mathrm{rk}$ we denote the rank of a free $\widehat{S}_{k}$-module or a free $\widehat{S}_{k}^{\emptyset}$-module.)

Proof. The first part of the theorem follows from the preceding proposition and the fact that $\mathbb{H}_{\widehat{T}}^{*}\left(F_{e}\right)=B_{e}$ as a $\widehat{\mathcal{Z}}_{k}$-module. Consider the localization map $\mathbb{H}_{\widehat{T}}^{*}(F) \rightarrow$ $\bigoplus_{x \in \widehat{\mathcal{W}}} \mathbb{H}_{\widehat{T}}^{*}\left(i_{x}^{*} F\right)$. Each $\mathbb{H}_{\widehat{T}}^{*}\left(i_{x}^{*} F\right)$ is naturally an $\widehat{S}_{k}$-module, and the right-hand side of the map above carries the obvious action of $\prod_{x \in \widehat{W}} \widehat{S}$. Moreover, the map itself is compatible with the actions of $\widehat{\mathcal{Z}}_{k}$ on the left-hand side and of $\prod_{x \in \widehat{\mathcal{W}}} \widehat{S}_{k}$ on the right, i.e. it is a $\widehat{\mathcal{Z}}_{k}$-module map. It induces an isomorphism

$$
\mathbb{H}_{\widehat{T}}^{*}(F) \otimes_{\widehat{S}_{k}} \widehat{S}_{k}^{\emptyset} \stackrel{\sim}{\rightarrow} \bigoplus_{x \in \widehat{\mathcal{W}}} \mathbb{H}_{\widehat{T}}^{*}\left(i_{x}^{*} F\right) \otimes_{\widehat{S}_{k}} \widehat{S}_{k}^{\emptyset},
$$

and the second claim follows.

7.5. Equivariant intersection cohomology sheaves. Let $\Pi^{-} \subset \mathscr{A}$ be the set of alcoves that are contained in the strip $H_{\alpha, 0}^{-} \cap H_{\alpha,-1}^{+}$for each simple root $\alpha$ (the set $-\Pi^{-}$is also called the "fundamental box"). Let $\widehat{w}_{0} \in \widehat{\mathcal{W}}$ be the element that corresponds to the smallest alcove $A_{\widehat{w}_{0}}$ in $\Pi^{-}$. Denote by $\mathscr{A}^{\circ} \subset \mathscr{A}$ the set of alcoves that correspond to the set $\widehat{\mathcal{W}}^{\circ}:=\left\{w \in \widehat{\mathcal{W}} \mid w \leq \widehat{w}_{0}\right\}$.

Definition 7.7. (1) Denote by $\mathcal{I}_{k}^{\circ} \subset \mathcal{I}_{k}$ the full subcategory that consists of direct sums of direct summands of objects of the form $\pi_{t}^{*} \pi_{t *} \circ \cdots \circ \pi_{s}^{*} \pi_{s *} F_{e}[n]$ for $s, \ldots, t \in \widehat{\mathcal{S}}$ such that $w=s \cdots t$ is a reduced expression for $w \in \widehat{\mathcal{W}}^{\circ}$.

(2) Analogously, denote by $\mathcal{H}_{k}^{\circ} \subset \mathcal{H}_{k}$ the full subcategory that consists of direct sums of direct summands of objects of the form $\theta^{t} \circ \cdots \circ \theta^{s} B_{e}[n]$ for $s, \ldots, t \in \widehat{\mathcal{S}}$ such that $w=s \cdots t$ is a reduced expression for $w \in \widehat{\mathcal{W}}^{\circ}$.

Since by Theorem 7.6 equivariant hypercohomology commutes with the translation functors on $\mathcal{I}_{k}$ and $\mathcal{H}_{k}$ we obtain an induced functor $\mathbb{H}_{\widehat{T}}^{*}: \mathcal{I}_{k}^{\circ} \rightarrow \mathcal{H}_{k}^{\circ}$.

Now suppose that $k$ is a field and denote by $\operatorname{IC}_{\widehat{T}, y} \in \mathrm{D}_{\widehat{T}}(\widehat{\mathcal{F}} 1, k)$ the equivariant intersection cohomology complex on the Schubert variety $\overline{\mathcal{O}_{y}}$ with coefficients in $k$. Choose $y \in \widehat{\mathcal{W}}^{\circ}$ and fix a reduced expression $y=s \cdots t$. If char $k=0$, then the decomposition theorem (cf. [BBD82]) together with some orbit combinatorics shows that

$$
\pi_{t}^{*} \pi_{t *} \circ \cdots \circ \pi_{s}^{*} \pi_{s *} F_{e} \cong \bigoplus_{i} \mathrm{IC}_{\widehat{T}, y_{i}}\left[n_{i}\right]
$$

for some $y_{i} \in \widehat{\mathcal{W}}^{\circ}$ and $n_{i} \in \mathbb{Z}$. Moreover, there is exactly one $i$ with $y_{i}=y$. By an equivariant version of a theorem of Kazhdan and Lusztig (cf. [KL80]) we have $\operatorname{rk} \mathbb{H}_{\widehat{T}}^{*}\left(i_{x}^{*} \mathrm{IC}_{\widehat{T}, y}\right)=h_{x, y}(1)$, again under the assumption char $k=0$.

Keep the reduced expression $s \cdots t$ fixed. Since the object $\pi_{t}^{*} \pi_{t *} \circ \cdots \circ \pi_{s}^{*} \pi_{s *} F_{e}$ as well as the intersection cohomology sheaves on Schubert varieties can be defined over $\mathbb{Z}$, we deduce that the above decomposition, as well as the character formula 
of Kazhdan and Lusztig, also holds if char $k$ is big enough, i.e. bigger than a certain number $N$ depending on the reduced expression.

Now in the definition of $\mathcal{I}_{k}^{\circ}$ only finitely many reduced expressions $s \cdots t$ occur. So we can deduce the following.

Theorem 7.8. Suppose char $k$ is either zero or big enough, i.e. bigger than a certain number $N$ depending on the root system $R$. Then the following holds.

(1) $\mathcal{I}_{k}^{\circ}$ is the full subcategory of $D_{\widehat{T}}(\widehat{\mathcal{F} 1}, k)$ that consists of objects isomorphic to a direct sum of shifted equivariant intersection cohomology sheaves $\mathrm{IC}_{\widehat{T}, y}$ with $y \in \widehat{\mathcal{W}}^{\circ}$.

(2) If $y \in \widehat{\mathcal{W}}^{\circ}$ and $y=s \cdots t$ is a reduced expression, then $\mathrm{IC}_{\widehat{T}, y}$ occurs, up to a shift, as the unique indecomposable direct summand in $\pi_{t}^{*} \pi_{t *} \cdots \pi_{s}^{*} \pi_{s *} F_{e}$ that is supported on $\overline{\mathcal{O}_{y}}$.

(3) For $y \in \widehat{\mathcal{W}}^{\circ}$ and $x \in \widehat{\mathcal{W}}$ we have $\operatorname{rk} \mathbb{H}_{\widehat{T}}^{*}\left(i_{x}^{*} \operatorname{IC}_{\widehat{T}, y}\right)=h_{x, y}(1)$.

\section{Modular Representations}

Let $k$ be an algebraically closed field of characteristic $p>h$ and let $\mathfrak{g}^{\vee}$ be the simple $k$-Lie algebra with root system $R^{\vee}$. Let $\mathfrak{h}^{\vee} \subset \mathfrak{b}^{\vee} \subset \mathfrak{g}^{\vee}$ be a Cartan and a Borel subalgebra. In the following we recall the definition and the main properties of a certain category $\mathcal{C}_{k}$ of $X^{\vee}$-graded restricted representations of $\mathfrak{g}^{\vee}$ that is equivalent to the category of rational representations of the subgroup scheme $G_{1}^{\vee} T^{\vee} \subset G^{\vee}$ that is generated by the (first) Frobenius kernel $G_{1}^{\vee} \subset G^{\vee}$ and the torus $T^{\vee}$. Our basic references for the following are the books [Jan03] and [AJS94].

8.1. Restricted representations of $\mathfrak{g}^{\vee}$. Denote by $U(\mathfrak{l})$ the universal enveloping algebra of a Lie algebra $\mathfrak{l}$. Recall that if $\mathfrak{l}$ is the Lie algebra of an algebraic group over a field of characteristic $p>0$, then $\mathfrak{l}$ is a $p$-Lie algebra, i.e. it is endowed with a $p$-th power map $D \mapsto D^{[p]}$. For each such Lie algebra let $U^{r e s}(\mathfrak{l})$ be the restricted enveloping algebra, i.e. the quotient of $U(\mathfrak{l})$ by the two-sided ideal generated by all $D^{p}-D^{[p]}$ with $D \in \mathfrak{l}$ (here $D^{p}$ denotes the $p$-th power of $D$ in $U(\mathfrak{l})$ ).

Set $U^{r e s}:=U^{r e s}\left(\mathfrak{g}^{\vee}\right)$. Then $U^{\text {res }}$ is an $X^{\vee}$-graded Lie algebra, where the grading comes from the adjoint action of the torus $T^{\vee}$. Let $U^{r e s}-\bmod _{X} \vee$ be the category of $X^{\vee}$-graded $U^{r e s}$-modules $M=\bigoplus_{\nu \in X^{\vee}} M_{\nu}$. For $\lambda \in \operatorname{Hom}\left(T^{\vee}, k^{\times}\right)$we denote by $\bar{\lambda} \in \operatorname{Hom}\left(\mathfrak{h}^{\vee}, k\right)$ its differential. As in [AJS94, Soe95] we define the full subcategory $\mathcal{C}_{k}$ of $U^{r e s}-\bmod _{X} \vee$ by the following condition on its objects:

$$
\mathcal{C}_{k}:=\left\{\begin{array}{l|c}
M \in U^{r e s}-\bmod _{X \vee} & \begin{array}{c}
M \text { is finitely generated, } \\
H m=\bar{\nu}(H) m \\
\forall H \in \mathfrak{h}^{\vee}, \nu \in X^{\vee}, m \in M_{\nu}
\end{array}
\end{array}\right\} .
$$

It is known that this category is equivalent to the category of finite-dimensional rational representations of $G_{1}^{\vee} T^{\vee}$ (cf. [Jan03]).

For each $\lambda \in X^{\vee}$ there is a standard module

$$
Z(\lambda):=U^{r e s}\left(\mathfrak{g}^{\vee}\right) \otimes_{U\left(\mathfrak{b}^{\vee}\right)} k_{\lambda}
$$

in $\mathcal{C}_{k}$, the baby Verma module with highest weight $\lambda$, which has a unique simple quotient $L(\lambda)$. The $L(\lambda)$ 's form a system of representatives for the simple objects in $\mathcal{C}_{k}$, and we denote by $[Z(\lambda): L(\mu)]$ the multiplicity of $L(\mu)$ in a Jordan-Hölder series of $Z(\lambda)$. This is a finite number, and for fixed $\lambda$ it is nonzero only for finitely many $\mu$. 
8.2. Deformed projective objects. There is a projective cover $P(\mu)$ of each simple object $L(\mu)$ in $\mathcal{C}_{k}$. Each $P(\mu)$ admits a Verma flag, i.e. a filtration such that the subquotients are isomorphic to various $Z(\lambda)$. The corresponding multiplicity $(P(\mu): Z(\lambda))$ is independent of the filtration and we have the Brauer-Humphreys reciprocity formula

$$
(P(\mu): Z(\lambda))=[Z(\lambda): L(\mu)] .
$$

Recall that we defined the algebra $S_{k}=S\left(X \otimes_{\mathbb{Z}} k\right)$ that we can now identify with $S\left(\mathfrak{h}^{\vee}\right)$. The deformed version $\widetilde{\mathcal{C}}_{k}$ of $\mathcal{C}_{k}$ carries an action of the completion $\tilde{S}_{k}$ of $S_{k}$ at the maximal ideal generated by $\mathfrak{h}^{\vee} \subset S_{k}$. We denote by $\tau: S_{k} \rightarrow \tilde{S}_{k}$ the canonical map. Following [AJS94, Section 1.2], we let $U$ be the quotient of $U\left(\mathfrak{g}^{\vee}\right)$ by the two-sided ideal generated by all $D^{[p]}-D^{p}$ with $D \in \mathfrak{g}_{\alpha \vee}^{\vee}$ for some $\alpha^{\vee} \in R^{\vee}$ (note that $U^{\text {res }}$ is a quotient of $U$ ). We consider $U \otimes_{k} \tilde{S}_{k}$ as an $X^{\vee}$-graded $\tilde{S}_{k}$-algebra and define $U \otimes_{k} \tilde{S}_{k}$-mod ${ }_{X \vee}$ as the category of $X^{\vee}$-graded $U \otimes_{k} \tilde{S}_{k}$-modules. Then $\widetilde{\mathcal{C}_{k}}$ is the full subcategory of $U \otimes_{k} \tilde{S}_{k}$-mod $X^{\vee}$ whose objects satisfy the following condition:

$$
\widetilde{\mathcal{C}}_{k}:=\left\{\begin{array}{l|c}
M \in U \otimes_{k} \tilde{S}_{k^{-}-\bmod _{X} \vee} \mid \begin{array}{c}
M \text { is finitely generated, } \\
(H \otimes 1) m=(\bar{\nu}(H) \otimes 1+1 \otimes H) m \\
\forall H \in \mathfrak{h}^{\vee}, \nu \in X^{\vee}, m \in M_{\nu}
\end{array}
\end{array}\right\} .
$$

For $\lambda \in X^{\vee}$ let $\tilde{S}_{k, \lambda}$ be the $\tilde{S}_{k}$-module that is free of rank one and on which $U\left(\mathfrak{b}^{\vee}\right)$ acts via the map $U\left(\mathfrak{b}^{\vee}\right) \rightarrow U\left(\mathfrak{h}^{\vee}\right)=S_{k} \stackrel{\lambda+\tau}{\rightarrow} \tilde{S}_{k}$. Then we can construct the deformed standard module

$$
\widetilde{Z}(\lambda):=U \otimes_{U\left(\mathfrak{b}^{\vee}\right)} \tilde{S}_{k, \lambda} .
$$

It is an object of $\widetilde{\mathcal{C}_{k}}$. For each $\mu \in X^{\vee}$ there is a deformed indecomposable projective object $\widetilde{P}(\mu)$ in $\widetilde{\mathcal{C}_{k}}$ that maps surjectively to $\widetilde{Z}(\mu)$. Again these projectives admit a finite filtration with subquotients isomorphic to deformed standard modules, and for the multiplicities we have

$$
(\widetilde{P}(\mu): \widetilde{Z}(\lambda))=(P(\mu): Z(\lambda)) .
$$

8.3. Translation functors and special representations. Define the " $p$ "-action of $\widehat{\mathcal{W}}$ on $X^{\vee}$ as follows: for $w \in \widehat{\mathcal{W}}$ and $\lambda \in X^{\vee}$ set

$$
w \cdot{ }_{p} \lambda:=p w\left(p^{-1}(\lambda+\rho)\right)-\rho,
$$

where $\rho=(1 / 2) \cdot \sum_{\alpha \in R^{+, v}} \alpha$.

The translation principle implies that in order to know the Jordan-Hölder multiplicities of all standard modules it is sufficient to calculate the Jordan-Hölder multiplicities of the modules $Z\left(x \cdot{ }_{p} 0\right)$ for $x \in \widehat{\mathcal{W}}$. The linkage principle states that $L(\mu)$ occurs as a subquotient of $Z\left(x \cdot{ }_{p} 0\right)$ only if $\mu=y \cdot{ }_{p} 0$ for some $y \in \widehat{\mathcal{W}}$. By the above, the number $\left[Z\left(x \cdot{ }_{p} 0\right): L\left(y \cdot{ }_{p} 0\right)\right]$ equals the number $\left(\widetilde{P}\left(y \cdot{ }_{p} 0\right): \widetilde{Z}\left(x \cdot{ }_{p} 0\right)\right)$ for all $x, y \in \widehat{\mathcal{W}}$. Moreover, an inherent $p X^{\vee}$-symmetry shows that it is sufficient to assume that $y \in \widehat{\mathcal{W}}^{\text {res, }-} \subset \widehat{\mathcal{W}}^{\circ}$, where $\widehat{\mathcal{W}}^{\text {res,- }}$ is the set of elements in $\widehat{\mathcal{W}}$ that correspond to alcoves in the anti-fundamental box $\Pi^{-}$.

Let us denote by $\widetilde{\mathcal{C}}_{k, 0}^{V} \subset \widetilde{\mathcal{C}}_{k}$ the full subcategory of objects in the principal block that admit a Verma flag, i.e. a filtration with subquotients isomorphic to standard modules of the form $\widetilde{Z}\left(w \cdot{ }_{p} 0\right)$ for various $w \in \widehat{\mathcal{W}}$. For $M \in \widetilde{\mathcal{C}}_{k, 0}^{V}$ we denote by 
supp $M$ its support, i.e. the multiset of $w \in \widehat{\mathcal{W}}$ such that $\widetilde{Z}\left(w \cdot{ }_{p} 0\right)$ occurs in a Verma flag of $M$ (so we count the $w$ in supp $M$ with multiplicity).

For each $s \in \widehat{\mathcal{S}}$ there is a translation functor $\theta^{s}: \widetilde{\mathcal{C}}_{k, 0}^{V} \rightarrow \widetilde{\mathcal{C}}_{k, 0}^{V}$. It has the following properties.

Proposition 8.1. (1) $\theta^{s}$ is an exact and selfadjoint functor.

(2) $\operatorname{supp} \theta^{s} M=\operatorname{supp} M \dot{\cup}(\operatorname{supp} M) s$.

(3) If $w=s \cdots t$ is a reduced expression, then $\widetilde{P}\left(w \cdot{ }_{p} 0\right)$ occurs as a direct summand in $\theta^{t} \cdots \theta^{s} \widetilde{P}(0)$ with multiplicity one.

Definition 8.2. The category of special deformed modular representations is the full subcategory $\mathcal{R}_{k}$ of $\widetilde{\mathcal{C}}_{k, 0}^{V}$ that consists of all objects that are isomorphic to a direct summand of a direct sum of objects of the form $\theta^{s} \cdots \theta^{t} \widetilde{Z}(0)$ for an arbitrary sequence $s, \ldots, t \in \widehat{\mathcal{S}}$.

8.4. A construction of deformed projectives. Denote by $w_{0} \in \mathcal{W}$ the longest element in the finite Weyl group. Note that it is the smallest element (in the Bruhat order) in $\widehat{\mathcal{W}}^{r e s,-}$. Let $y=s_{1} \cdots s_{n}$ be a reduced expression for $y \in \widehat{\mathcal{W}}^{r e s,-}$. We say that this is a reduced expression through $w_{0}$ if $w_{0}=s_{1} \cdots s_{l\left(w_{0}\right)}$. The following proposition shows that all the deformed objects that we are interested in actually appear in $\mathcal{R}_{k}$.

Proposition 8.3. Let $y \in \widehat{\mathcal{W}}^{r e s},-$ and choose a reduced expression $y=s \cdots t$ through $w_{0}$. Then $\widetilde{P}\left(y \cdot{ }_{p} 0\right)$ is the unique indecomposable direct summand in $\theta^{t} \ldots$ $\theta^{s} \widetilde{Z}(0)$ that contains $y$ in its support.

Proof. It is enough to prove the claim for $y=w_{0}$, since the general case follows from this by Proposition 8.1. (3).

So let $w_{0}=s \cdots t$ be a reduced expression. Consider a surjection $\widetilde{P}(0) \rightarrow \widetilde{Z}(0)$. Proposition 8.1 shows that we get a surjection $\theta^{t} \cdots \theta^{s} \widetilde{P}(0) \rightarrow \theta^{t} \cdots \theta^{s} \widetilde{Z}(0)$ and that the module on the left-hand side contains exactly one direct summand which is isomorphic to $\widetilde{P}\left(w_{0} \cdot p 0\right)$.

Since $s, \ldots, t \in \mathcal{W}$ we can deduce the following from Proposition 8.1

$$
\left(\theta^{t} \cdots \theta^{s} \widetilde{Z}(0): \widetilde{Z}\left(w \cdot{ }_{p} 0\right)\right)= \begin{cases}\left(\theta^{t} \cdots \theta^{s} \widetilde{P}(0): \widetilde{Z}\left(w \cdot{ }_{p} 0\right)\right) & \text { if } w \in \mathcal{W} \\ 0 & \text { if } w \notin \mathcal{W} .\end{cases}
$$

Each direct summand $P$ of $\theta^{t} \cdots \theta^{s} \widetilde{P}(0)$ has a Verma flag. By Corollary 9.6 of AJS94 we can find a Verma flag that starts with all subquotients $\widetilde{Z}\left(w \cdot{ }_{p} 0\right)$ such that $w \npreceq e$. From the following Lemma 8.4 we deduce that this Verma flag ends with all $\widetilde{Z}\left(w \cdot{ }_{p} 0\right)$ with $w \in \mathcal{W}$. From the above multiplicity result we deduce that the restriction of the map $\theta^{t} \cdots \theta^{s} \widetilde{P}(0) \rightarrow \theta^{t} \cdots \theta^{s} \widetilde{Z}(0)$ to $P$ is the quotient map modulo the biggest submodule admitting a Verma flag with subquotients $\widetilde{Z}\left(w \cdot{ }_{p} 0\right)$ and $w \notin \mathcal{W}$.

Hence the direct sum decomposition of $\theta^{t} \cdots \theta^{s} \widetilde{P}(0)$ induces a direct sum decomposition in $\theta^{t} \cdots \theta^{s} \widetilde{Z}(0)$. Since the support of $\widetilde{P}\left(w_{0} \cdot{ }_{p} 0\right)$ coincides with $\mathcal{W}$ (even with multiplicities), the proposition is proved.

Lemma 8.4. Choose $s, \ldots, t \in \mathcal{S}$ such that $w_{0}=s \cdots t$ is a reduced expression. If $w \in \operatorname{supp} \theta^{t} \cdots \theta^{s} \widetilde{P}(0)$ is such that $w \preceq e$, then $w \in \mathcal{W}$. 
Proof. Let us denote by $\Lambda^{\prime} \subset \widehat{\mathcal{W}}$ the support of $\widetilde{P}(0)$ and by $\Lambda \subset \widehat{\mathcal{W}}$ the support of $\theta^{t} \cdots \theta^{s} \widetilde{P}(0)$, both taken without multiplicities. Then $\Lambda=\Lambda^{\prime} \cdot \mathcal{W}$.

The set $\Lambda$ is partially ordered by the generic Bruhat order. By definition, there is $\lambda \in \mathbb{N} R^{\vee,+}$ such that the map $\Lambda \rightarrow \widehat{\mathcal{W}}, w \mapsto t_{\lambda} w$, preserves the order (here $\widehat{\mathcal{W}}$ carries the ordinary Bruhat order). Moreover, this map is equivariant with respect to the multiplication action of $\mathcal{W}$ on the right.

Now $\mathcal{W} \subset \widehat{\mathcal{W}}$ is a parabolic subgroup, which implies that there is an induced (Bruhat) order on $\widehat{\mathcal{W}} / \mathcal{W}$ such that $\widehat{\mathcal{W}} \rightarrow \widehat{\mathcal{W}} / \mathcal{W}$ is a map between partially ordered sets. Together with the results in the preceding paragraph we deduce that there is a partial order on $\Lambda / \mathcal{W}$ such that the map $\Lambda \rightarrow \Lambda / \mathcal{W}$ respects the orders (explicitly, we identify $\Lambda / \mathcal{W}$ with its image under the composition $\Lambda \stackrel{t_{\lambda}}{\rightarrow} \widehat{\mathcal{W}} \rightarrow \widehat{\mathcal{W}} / \mathcal{W}$ and take the induced order). Let us fix this order on $\Lambda / \mathcal{W}$.

The maps $\Lambda^{\prime} \rightarrow \Lambda \rightarrow \Lambda / \mathcal{W}$ respect the partial orders and the composition is surjective. Since $e$ is the smallest element in $\Lambda^{\prime}$, its image $\bar{e}$ in $\Lambda / \mathcal{W}$ is the smallest element in $\Lambda / \mathcal{W}$. Hence each element in $\Lambda$ that is smaller than $e$ must be mapped to $\bar{e}$, hence lies in the $\mathcal{W}$-orbit of $e$.

8.5. The quantum group case. In Lus90] Lusztig constructs the restricted quantum group $\mathbf{u}$ associated to the root system $R$, its set of positive roots $R^{+} \subset R$, an integer $l$ which is prime to the entries of the Cartan matrix of $R$, and a primitive $l$-th root of unity $\zeta$. The quantum group is a finite-dimensional $\mathbb{Z} R$-graded algebra over the field $k=\mathbb{Q}(\zeta)$.

There is a complete analog of the above definitions and structures for the representation theory of $\mathbf{u}$. In particular, one can define standard modules $Z(\lambda)$, simple modules $L(\lambda)$ and projective objects $P(\lambda)$ (in a certain category). The standard and the projective objects admit deformed versions and we can define a category $\mathcal{R}_{\mathbb{Q}(\zeta)}$ that describes the representation theory of $\mathbf{u}$. (For an overview, see And95, Soe95.)

8.6. The Andersen-Jantzen-Soergel equivalence. Recall the definition of $\mathcal{M}_{k}^{\prime}$ in Section 6. In order to compare the combinatorial category to the category $\mathcal{R}_{k}$ we need to extend scalars. So let $\widetilde{\mathcal{M}}_{k}^{\prime}$ be the combinatorial category defined in exactly the same way as $\mathcal{M}_{k}^{\prime}$, but with the algebra $S_{k}$ replaced by $\tilde{S}_{k}$. Then there is an obvious extension of scalar functors $\cdot \otimes_{S_{k}} \tilde{S}_{k}: \mathcal{M}_{k}^{\prime} \rightarrow \widetilde{\mathcal{M}}_{k}^{\prime}$ that naturally commutes with the translation functors.

The following is one of the main results in AJS94.

Theorem 8.5. Suppose that $k=\mathbb{Q}(\zeta)$ or char $k=p>h$. Then there is an equivalence

$$
\mathbb{V}: \mathcal{R}_{k} \stackrel{\sim}{\rightarrow} \widetilde{\mathcal{M}}_{k}^{\prime}
$$

such that $\mathbb{V} \circ \theta^{s} \cong \mathcal{T}^{\prime s} \circ \mathbb{V}$ for all $s \in \widehat{\mathcal{S}}$, and such that for all $M \in \mathcal{R}_{k}$ and $x \in \widehat{\mathcal{W}}$ we have

$$
\left(M: \widetilde{Z}\left(x \cdot{ }_{p} 0\right)\right)=\operatorname{rk} \mathbb{V}(M)\left(A_{x}\right) .
$$

The following partly proves the conjectures of Lusztig.

Theorem 8.6. Suppose that $k=\mathbb{Q}(\zeta)$ or that char $k=p$ is large enough. For $x \in \widehat{\mathcal{W}}$ and $y \in \widehat{\mathcal{W}}^{\text {res },-}$ we have

$$
\left[Z\left(x \cdot{ }_{p} 0\right): L\left(y \cdot{ }_{p} 0\right)\right]=p_{w_{0} x, w_{0} y}(1) .
$$


Proof. By the results in Section 8.2 the claim is equivalent to $\left(\widetilde{P}\left(y \cdot{ }_{p} 0\right): \widetilde{Z}\left(x \cdot{ }_{p} 0\right)\right)=$ $p_{w_{0} x, w_{0} y}(1)$. Consider the composition

$$
\Phi: \mathcal{I}_{k} \stackrel{\mathbb{H}_{\widehat{T}}^{*}}{\longrightarrow} \mathcal{H}_{k} \stackrel{\Psi}{\longrightarrow} \mathcal{M}_{k} \cong \mathcal{M}_{k}^{\prime} \stackrel{\cdot \otimes_{S_{k}} \tilde{S}_{k}}{\longrightarrow} \widetilde{\mathcal{M}}_{k}^{\prime} \stackrel{\mathbb{V}^{-1}}{\longrightarrow} \mathcal{R}_{k} .
$$

Using Theorem 7.6] Theorem 5.4 and Theorem 8.5 we deduce that $\Phi \circ \pi_{s}^{*} \pi_{s *} \cong \theta^{s} \circ \Phi$ and that

$$
\left(\Phi(F): \widetilde{Z}\left(x \cdot \cdot_{p} 0\right)\right)=\operatorname{rk} \mathbb{H}_{\widehat{T}}^{*}\left(i_{x}^{*} F\right)
$$

for all $F \in \mathcal{I}_{k}$.

Let $y \in \widehat{\mathcal{W}}^{r e s,-}$ and choose a reduced expression $y=s \cdots t$ through $w_{0}$. By Proposition 8.3 the object $\widetilde{P}\left(y \cdot{ }_{p} 0\right)$ occurs as the unique indecomposable direct summand of $\theta^{t} \circ \cdots \circ \theta^{s} \widetilde{Z}(0)$ that contains $y$ in its support. Suppose that $k=\mathbb{Q}(\zeta)$ or that char $k=p$ is large enough such that the assumptions in Theorem 7.8 hold. Then, on the geometric side, the sheaf $\pi_{t}^{*} \pi_{t *} \cdots \pi_{s}^{*} \pi_{s *}\left(F_{e}\right)$ decomposes into a direct sum of shifted equivariant intersection cohomology complexes on Schubert varieties, and $\mathrm{IC}_{\widehat{T}, y}$ occurs with multiplicity one. Moreover, $\operatorname{rk} \mathbb{H}_{\widehat{T}}^{*}\left(i_{x}^{*} \mathrm{IC}_{\widehat{T}, y}\right)=h_{x, y}(1)$. From the above we deduce that $\widetilde{P}\left(y \cdot_{p} 0\right)$ is a direct summand of $\Phi\left(\mathrm{IC}_{\widehat{T}, y}\right)$; hence

$$
\left(\widetilde{P}\left(y \cdot{ }_{p} 0\right): \widetilde{Z}\left(x \cdot{ }_{p} 0\right)\right) \leq h_{x, y}(1) .
$$

By a result of Lusztig in Lus80a we have $h_{x, y}(1)=p_{w_{0} x, w_{0} y}(1)$.

Now suppose that the theorem is proven for $y \in \widehat{\mathcal{W}}^{r e s,-}$ and choose $s \in \widehat{\mathcal{S}}$ with $y s \in \widehat{\mathcal{W}}^{r e s,-}$ and $y<y s$, if possible. By AJS94, pp. 238/239, the decomposition

of $\theta^{s} \widetilde{P}\left(y \cdot{ }_{p} 0\right)$ into indecomposables is at most as the Lusztig conjecture predicts; i.e., we have

$$
\left(\widetilde{P}\left(y s \cdot{ }_{p} 0\right): \widetilde{Z}\left(x \cdot{ }_{p} 0\right)\right) \geq p_{w_{0} x, w_{0} y s}(1) .
$$

Hence we deduce $\left(\widetilde{P}\left(y s \cdot{ }_{p} 0\right): \widetilde{Z}\left(x \cdot{ }_{p} 0\right)\right)=p_{w_{0} x, w_{0} y s}(1)$, which is the claim for ys.

\section{Braden-MacPherson SHEAVES ON MOMENT GRAPHS}

In this final section we give an alternative construction of the objects in $\mathcal{H}_{k}^{\circ}$ as spaces of global sections of certain sheaves on a moment graph. This allows us to apply the results in [Fie06] and [Fie08c] in the representation-theoretic context.

9.1. GKM-pairs. Apart from char $k \neq 2$ and $\neq 3$ in case $R$ is of type $G_{2}$, the main restriction on $k$ is the GKM-property.

Definition 9.1. Let $\widehat{\mathcal{G}}^{\prime} \subset \widehat{\mathcal{G}}$ be a finite full subgraph. We say that $\left(\widehat{\mathcal{G}}^{\prime}, k\right)$ is a GKM-pair if the labels at two different edges of $\widehat{\mathcal{G}}^{\prime}$ that meet at a common vertex are linearly independent in $\widehat{X} \otimes_{\mathbb{Z}} k$.

For the application to Lusztig's conjecture we recover the usual restriction on $k$. Let $\widehat{\mathcal{G}}^{\circ}$ be the full subgraph of $\widehat{\mathcal{G}}$ with vertices in $\widehat{\mathcal{W}}^{\circ}=\left\{w \in \widehat{\mathcal{W}} \mid w \leq \widehat{w}_{0}\right\}$.

Lemma 9.2. Suppose that $\operatorname{char} k=0$ or $\operatorname{char} k=p \geq h$. Then $\left(\widehat{\mathcal{G}}^{\circ}, k\right)$ is a GKM-pair.

Proof. In the characteristic 0 case the pairwise linear independence of the positive affine roots implies the statement. So suppose that char $k$ is positive and that there are $\alpha, \beta \in R^{+}, m, n \in \mathbb{Z}$ such that $\overline{\alpha_{n}}=\overline{\alpha-n \delta}$ and $\overline{\beta_{m}}=\overline{\beta-m \delta}$ are linearly 
dependent in $\widehat{X} \otimes_{\mathbb{Z}} k$. This implies that $\bar{\alpha}=\alpha \otimes 1$ and $\bar{\beta}=\beta \otimes 1$ are linearly dependent in $X \otimes_{\mathbb{Z}} k$.

Now char $k \geq h$ implies that if $\bar{\alpha}$ and $\bar{\beta}$ are linearly dependent, then $\alpha=\beta$. Then $\overline{\alpha-n \delta}$ and $\overline{\alpha-m \delta}$ are linearly dependent if and only if $n-m$ is divisible by $p$.

Recall that we defined $\mathscr{A}^{\circ} \subset \mathscr{A}$ as the set of alcoves that correspond to the set $\widehat{\mathcal{W}}^{\circ}$. We claim that for each positive root $\gamma$, the set $\mathscr{A}^{\circ}$ is contained in $H_{\gamma, h}^{-} \cap H_{\gamma,-h}^{+}$. In order to prove this we choose an alcove $A \in \mathscr{A}^{\circ}$. Our claim is $\mathcal{W}$-invariant; hence we can assume that $A$ is contained in the anti-dominant cone, i.e. $A \subset H_{\nu, 0}^{-}$for all $\nu \in R^{+}$. In particular, $A \subset H_{\gamma, h}^{-}$.

In order to finish the proof of our claim above it is enough to show that the alcove $A_{\widehat{w}_{0}}$ corresponding to the largest element $\widehat{w}_{0} \in \widehat{\mathcal{W}}^{\circ}$ (with respect to the Bruhat order) is contained in $H_{\gamma,-h}^{+}$, since $A$ is larger (with respect to the generic order) than $A_{\widehat{w}_{0}}$. But this is a direct consequence of the definition of the Coxeter number.

We finish the proof of the lemma. Suppose that there is $w \in \widehat{\mathcal{W}}^{\circ}$ and $\alpha, \beta \in R^{+}$, $n, m \in \mathbb{Z}$ with $s_{\alpha, n} w, s_{\beta, m} w \in \widehat{\mathcal{W}}^{\circ}$ such that $\overline{\alpha_{n}}$ and $\overline{\beta_{m}}$ are linearly dependent. As we have shown above, this already implies that $\alpha=\beta$ and that $p$ divides $n-m$. We have to show that $m=n$ under the assumption $p \geq h$.

Since each alcove in $\mathscr{A}^{\circ}$ is contained in $H_{\alpha,-h}^{+} \cup H_{\alpha, h}^{-}$the assumptions $w, s_{\alpha, n} w$, $s_{\alpha, m} w \in \widehat{\mathcal{W}}^{\circ}$ imply that $|m-n|<h$, from which we deduce $m=n$.

9.2. Braden-MacPherson sheaves. Let $x$ be a vertex of $\widehat{\mathcal{G}}$ and $\mathscr{F}$ a sheaf on $\widehat{\mathcal{G}}$. Denote by $\widehat{\mathcal{G}}_{>x}$ the sub-moment graph with set of vertices $\{y \in \mathcal{V} \mid y>x\}$. The restriction of $\mathscr{F}$ to $\widehat{\mathcal{G}}_{>x}$ gives a sheaf $\mathscr{F}_{>x}$ on $\widehat{\mathcal{G}}_{>x}$. Define $\mathcal{V}_{\delta x} \subset \mathcal{V}$ as the set of vertices $y$ with $y>x$ and that are connected to $x$ by an edge. Accordingly, let $\mathcal{E}_{\delta x}=\left\{E: x-y \mid y \in \mathcal{V}_{\delta x}\right\} \subset \mathcal{E}$ be the corresponding set of edges. Denote by $\mathscr{F}^{\delta x}$ the image of the map

$$
\Gamma\left(\mathscr{F}_{>x}\right) \subset \prod_{y>x} \mathscr{F}^{y} \stackrel{p}{\rightarrow} \prod_{y \in \mathcal{V}_{\delta x}} \mathscr{F}^{y} \stackrel{\rho}{\rightarrow} \prod_{E \in \mathcal{E}_{\delta x}} \mathscr{F}^{E},
$$

where $p$ is the projection along the decomposition and $\rho=\prod_{y \in \mathcal{V}_{\delta x}} \rho_{y, E}$. Define

$$
\rho_{x, \delta x}:=\left(\rho_{x, E}\right)_{E \in \mathcal{E}_{\delta x}}^{T}: \mathscr{F}^{x} \rightarrow \prod_{E \in \mathcal{E}_{\delta x}} \mathscr{F}^{E}
$$

Theorem 9.3 ([BM01]; cf. also [Fie08a]). There is an up-to-isomorphism unique $k$-sheaf $\mathscr{B}_{w}$ on $\widehat{\mathcal{G}}$ with the following properties:

(1) $\mathscr{B}_{w}^{w} \cong \widehat{S}_{k}$.

(2) If $x \stackrel{\alpha}{-} y$ is an edge and $x<y$, then the map $\rho_{y, E}: \mathscr{B}_{w}^{y} \rightarrow \mathscr{B}_{w}^{E}$ is surjective with kernel $\alpha \cdot \mathscr{B}_{w}^{y}$.

(3) For any $x \in \mathcal{V}$, the image of $\rho_{x, \delta x}$ is $\mathscr{B}_{w}^{\delta x}$, and $\rho_{x, \delta x}: \mathscr{B}_{w}^{x} \rightarrow \mathscr{B}_{w}^{\delta x}$ is a projective cover in the category of graded $\widehat{S}_{k}$-modules.

We call the sheaf $\mathscr{B}_{w}$ the Braden-MacPherson sheaf on $\widehat{\mathcal{G}}$. It is called the canonical sheaf in BM01].

We set $B_{w}:=\Gamma\left(\mathscr{B}_{w}\right) \in \widehat{\mathcal{Z}}_{k}$-mod. 
Proposition 9.4. Suppose that $\operatorname{char} k=0$ or char $k=p \geq h$. Then each indecomposable object in $\mathcal{H}_{k}^{\circ}$ is, up to a shift in degree, isomorphic to the $\widehat{\mathcal{Z}}_{k}$-module $B_{x}$ for some vertex $x$ of $\widehat{\mathcal{G}}^{\circ}$.

Proof. We have to show that for each reduced expression $w=s \cdots t$ for $w \in \widehat{\mathcal{W}}^{\circ}$, the object $\theta^{t} \circ \cdots \circ \theta^{s} B_{e}$ is isomorphic to a direct sum of shifted copies of various $B_{x}$ with $x \in \widehat{\mathcal{W}}^{\circ}$. For this it is enough to prove that $\theta^{s} B_{w}$ decomposes in such a way for each $w \in \widehat{\mathcal{W}}^{\circ}, s \in \widehat{\mathcal{S}}$ with $w s \in \widehat{\mathcal{W}}^{\circ}$ and $w<w s$.

In the following we explain some arguments developed in [Fie08a, Fie08b]. Note that the following heavily depends on the GKM-property. In Fie08a we associated to each subgraph $\widehat{\mathcal{G}}^{\prime}$ the full subcategory $\mathcal{V}\left(\widehat{\mathcal{G}}^{\prime}\right)_{k}$ of $\widehat{\mathcal{Z}}_{k}$-mod that consists of modules that admit a Verma flag and we defined on $\mathcal{V}\left(\widehat{\mathcal{G}}^{\prime}\right)_{k}$ a nonstandard exact structure. We showed in Fie08b] that the indecomposable projective objects in $\mathcal{V}\left(\widehat{\mathcal{G}}^{\prime}\right)_{k}$ are, up to shifts, represented by the set $\left\{B_{x}\right\}$, where $x$ is a vertex of $\widehat{\mathcal{G}}^{\prime}$.

We also showed in $\left[\right.$ Fie08b] that, if $w<w s$, the functor $\theta^{s}: \widehat{\mathcal{Z}}_{k}(\leq w s)$-mod $\rightarrow$ $\widehat{\mathcal{Z}}_{k}(\leq w s)$-mod preserves $\mathcal{V}\left(\widehat{\mathcal{G}}_{\leq w s}\right)_{k}$ and is exact and selfadjoint; hence it also preserves the subcategory of projective objects. In particular, we deduce that $\theta^{s} B_{w}$ is projective in $\mathcal{V}\left(\widehat{\mathcal{G}}_{\leq w s}\right)_{k}$ and hence decomposes into a direct sum of shifted copies of some $B_{x}$.

9.3. A conjecture. Let $w \in \widehat{\mathcal{W}}$ be arbitrary. We can then consider the full submoment graph $\widehat{\mathcal{G}}_{\leq w}$ of $\widehat{\mathcal{G}}$ that contains all vertices that are smaller or equal to $w$.

Conjecture 9.5. Let $w \in \widehat{\mathcal{W}}$ and suppose that $\left(\widehat{\mathcal{G}}_{\leq w}, k\right)$ is a GKM-pair. Then for all $x \in \widehat{\mathcal{W}}$ we have

$$
\operatorname{rk} \mathscr{B}_{w}^{x}=h_{x, w}(1) .
$$

By the results in the previous sections, this conjecture implies Lusztig's modular and quantum conjectures. By [Fie08a, it also implies the Kazhdan-Lusztig conjecture on the simple highest weight characters of Kac-Moody algebras.

9.4. The smooth locus of a moment graph. In Fie08a we determined the "smooth locus" of moment graphs for which the intersection sheaf is self-dual. In our situation we obtain a proof of the multiplicity-one case of Conjecture 9.5 .

Theorem 9.6. Let $w \in \widehat{\mathcal{W}}$ and suppose that $\left(\widehat{\mathcal{G}}_{\leq w}, k\right)$ is a GKM-pair. Then we have, for all $x \in \widehat{\mathcal{W}}$,

$$
\operatorname{rk} \mathscr{B}_{w}^{x}=1 \text { if and only if } h_{x, w}(1)=1 .
$$

We obtain, as a special case, part (2) of Theorem 1.1 from the introduction:

Corollary 9.7. Suppose that $p>h$. For $y \in \widehat{\mathcal{W}}^{\text {res },-}$ and $x \in \widehat{\mathcal{W}}$ we have

$$
\left[Z\left(x \cdot{ }_{p} 0\right): L\left(y \cdot{ }_{p} 0\right)\right]=1 \text { if and only if } p_{w_{0} x, w_{0} y}(1)=1 .
$$

Proof. As in the proof of Theorem 8.6 we see that our claim is equivalent to

$$
\left(\widetilde{P}\left(y \cdot{ }_{p} 0\right): \widetilde{Z}\left(x \cdot{ }_{p} 0\right)\right)=1 \text { if and only if } p_{w_{0} x, w_{0} y}(1)=1 .
$$


It is known that $\left(\widetilde{P}\left(y \cdot{ }_{p} 0\right): \widetilde{Z}\left(x \cdot{ }_{p} 0\right)\right) \geq p_{w_{0} x, w_{0} y}(1)$ in any case. Since for $x \in \widehat{\mathcal{W}}$, $y \in \widehat{\mathcal{W}}^{\circ}$ with $x \leq y$ one has $p_{w_{0} x, w_{0} y}(1) \geq 1$, we deduce that $\left(\widetilde{P}\left(y \cdot{ }_{p} 0\right): \widetilde{Z}\left(x \cdot{ }_{p} 0\right)\right)$ $=1$ implies that $p_{w_{0} x, w_{0} y}(1)=1$.

Now assume that $p_{w_{0} x, w_{0} y}(1)=h_{x, y}(1)=1$. We consider the composition

$$
\Phi^{\prime}: \mathcal{H}_{k} \stackrel{\Psi}{\longrightarrow} \mathcal{M}_{k} \cong \mathcal{M}_{k}^{\prime} \stackrel{\cdot \otimes_{S_{k}} \tilde{S}_{k}}{\longrightarrow} \widetilde{\mathcal{M}}_{k}^{\prime} \stackrel{\mathbb{V}^{-1}}{\longrightarrow} \mathcal{R}_{k}
$$

In a similar way as in the proof of Theorem 8.6 we deduce that $\widetilde{P}\left(y \cdot{ }_{p} 0\right)$ is a direct summand of $\Phi^{\prime}\left(B_{y}\right)$, and Theorem 9.6 then implies that

$$
\left(\widetilde{P}\left(y \cdot{ }_{p} 0\right): \widetilde{Z}\left(x \cdot{ }_{p} 0\right)\right) \leq 1 .
$$

The reverse inequality is stated above; hence $\left(\widetilde{P}\left(y \cdot r_{p} 0\right): \widetilde{Z}\left(x_{p_{p}} 0\right)\right)=1$.

9.5. An upper bound on the exceptional primes. Let $\mathbf{s}=\left(s_{1}, \ldots, s_{l}\right)$ be a sequence in $\widehat{\mathcal{S}}$ of length $l$. Let us define the corresponding Bott-Samelson element $\underline{H}(\mathbf{s})$ in the affine Hecke algebra by

$$
\underline{H}(\mathbf{s}):=\underline{H}_{s_{1}} \cdots \underline{H}_{s_{l}} .
$$

Let the polynomials $a_{x} \in \mathbb{Z}[v]$ be defined by

$$
\underline{H}(\mathbf{s})=\sum_{x \in \widehat{\mathcal{W}}} a_{x} \tilde{T}_{x} .
$$

Set $r_{x}=a_{x}(1)$ and set

$$
r=r(\mathbf{s}):=\max _{x}\left\{r_{x}\right\} .
$$

Let $d_{x}=\left(\frac{d}{d v} a_{x}\right)(1)$ be the sum of the exponents of $a_{x}$, and set

$$
d=d(\mathbf{s}):=\max _{x}\left\{d_{x}\right\} .
$$

We denote by $\widehat{R}_{\mathbf{s}}^{+} \subset \widehat{R}^{+}$the subset of all positive roots that appear as a label on the subgraph of $\widehat{\mathcal{G}}$ that contains all vertices that are smaller or equal to a subword of $s_{1} \cdots s_{l}$. We define the height ht $(\alpha)$ of a positive affine root $\alpha \in \widehat{R}^{+}$as the number $n$ such that $\alpha$ can be written as a sum of $n$ elements of $\widehat{\Pi}$ and we set

$$
N=N(\mathbf{s}):=\max _{\alpha \in \widehat{R}_{\mathbf{s}}^{+}}\{\mathrm{ht}(\alpha)\}
$$

Now we associate to $\mathbf{s}$ the number $U(\mathbf{s}):=r !\left(r !(r-1) ! N^{l+2 d}\right)^{r}$ and set, for $w \in \widehat{\mathcal{W}}$

$$
U(w):=\min _{\mathbf{s}} U(\mathbf{s})
$$

where the minimum is taken over all sequences $\mathbf{S}$ such that $s_{1} \cdots s_{l}$ is a reduced expressions for $w$. Note that $U\left(w^{\prime}\right) \leq U(w)$ for $w^{\prime} \leq w$. The main result in [Fie08c] is the following:

Theorem 9.8. Let $w \in \widehat{\mathcal{W}}$ and suppose that char $k=p>U(w)$. Then Conjecture 9.5 holds for the moment graph $\widehat{\mathcal{G}}_{\leq w}$.

We now obtain part (3) of Theorem 1.1 from the introduction:

Corollary 9.9. Suppose that $p>U\left(\widehat{w}_{0}\right)$. For $x \in \widehat{\mathcal{W}}$ and $y \in \widehat{\mathcal{W}}^{\text {res },-}$ we have

$$
\left[Z\left(x \cdot{ }_{p} 0\right): L\left(y \cdot{ }_{p} 0\right)\right]=p_{w_{0} x, w_{0} y}(1) .
$$




\section{ACKNowledgements}

I thank Henning Haahr Andersen, Jens Carsten Jantzen, Masaharu Kaneda, Olaf Schnürer, Wolfgang Soergel and Geordie Williamson for helpful remarks on various versions of this paper.

\section{REFERENCES}

[ABG04] Sergey Arkhipov, Roman Bezrukavnikov, and Victor Ginzburg, Quantum groups, the loop Grassmannian, and the Springer resolution, J. Amer. Math. Soc. 17 (2004), no. 3, 595-678. MR.2053952 (2005g:16055)

[AJS94] Henning Haahr Andersen, Jens Carsten Jantzen, and Wolfgang Soergel, Representations of quantum groups at a pth root of unity and of semisimple groups in characteristic $p$ : independence of p, Astérisque (1994), no. 220, 321 pp. MR.1272539 (95j:20036)

[And95] Henning Haahr Andersen, The irreducible characters for semi-simple algebraic groups and for quantum groups, Proceedings of the International Congress of Mathematicians, Vols. 1, 2 (Zürich, 1994) (Basel), Birkhäuser, 1995, pp. 732-743. MR1403973 (97m:20051)

[BBD82] Alexander Beilinson, Joseph Bernstein, and Pierre Deligne, Faisceaux pervers, Analysis and topology on singular spaces, I (Luminy, 1981), Astérisque, vol. 100, Soc. Math. France, Paris, 1982, pp. 5-171. MR751966 (86g:32015)

[BGS96] Alexander Beilinson, Victor Ginzburg, and Wolfgang Soergel, Koszul duality patterns in representation theory, J. Amer. Math. Soc. 9 (1996), no. 2, 473-527. MR1322847 (96k:17010)

[BMR08] Roman Bezrukavnikov, Ivan Mirković, and Dmitriy Rumynin, Localization of modules for a semisimple Lie algebra in prime characteristic, Ann. of Math. (2), 167, no. 3, 2008, 945-991. MR 2415389 (2009e:17031)

[BM01] Tom Braden and Robert MacPherson, From moment graphs to intersection cohomology, Math. Ann. 321 (2001), no. 3, 533-551. MR1871967|(2003g:14030)

[Fie08a] Peter Fiebig, Sheaves on moment graphs and a localization of Verma flags, Adv. Math. 217 (2008), 683-712. MR2370278 (2008m:17044)

[Fie08b] - The combinatorics of Coxeter categories, Trans. Amer. Math. Soc. 360 (2008), 4211-4233. MR.2395170 (2009g:20087)

[Fie06] - The multiplicity one case of Lusztig's conjecture, Duke Math. J. 153 (2010), no. $3,551-571$.

[Fie07] Lusztig's conjecture as a moment graph problem, to appear in Bull. London Math. Soc., preprint arXiv:0712.3909 (2007).

[Fie08c] - An upper bound on the exceptional characteristics for Lusztig's character formula, preprint arXiv:0811.1674 (2008).

[FW] Peter Fiebig and Geordie Williamson, Parity sheaves, moment graphs and the p-smooth locus of Schubert varieties, preprint 2010, arXiv:1008.0719.

[FG06] Edward Frenkel and Dennis Gaitsgory, Local geometric Langlands correspondence and affine Kac-Moody algebras, Algebraic geometry and number theory, Progr. Math., vol. 253, Birkhäuser Boston, Boston, MA, 2006, pp. 69-260. MR2263193(2008e:17023)

[GKM98] Mark Goresky, Robert Kottwitz, and Robert MacPherson, Equivariant cohomology, Koszul duality, and the localization theorem, Invent. Math. 131 (1998), no. 1, 25-83. MR:1489894 (99c:55009)

[Hum71] James E. Humphreys, Modular representations of classical Lie algebras and semisimple groups, J. Algebra 19 (1971), 51-79. MR0283038(44:271)

[Hum90] James E. Humphreys, Reflection Groups and Coxeter Groups, Cambridge Studies in Advanced Mathematics 29, Cambridge University Press, 1990. MR1066460|(92h:20002)

[Jan03] Jens Carsten Jantzen, Representations of algebraic groups, second ed., Mathematical Surveys and Monographs, vol. 107, American Mathematical Society, Providence, RI, 2003. MR2015057 (2004h:20061)

[Kat85] Shin-ichi Kato, On the Kazhdan-Lusztig polynomials for affine Weyl groups, Adv. Math. 55 (1985), no. 2, 103-130. MR772611(86d:20048)

[KL79] David Kazhdan and George Lusztig, Representations of Coxeter groups and Hecke algebras, Invent. Math. 53 (1979), no. 2, 165-184. MR560412 (81j:20066) 
[KL80] Schubert varieties and Poincaré duality, Geometry of the Laplace operator (Proc. Sympos. Pure Math., Univ. Hawaii, Honolulu, Hawaii, 1979), Proc. Sympos. Pure Math., XXXVI, Amer. Math. Soc., Providence, R.I., 1980, pp. 185-203. MR573434 (84g:14054)

[KL93] , Tensor structures arising from affine Lie algebras. I-IV, J. Amer. Math. Soc. 6 (1993), 905-947; J. Amer. Math. Soc. 6 (1993), 949-1011; J. Amer. Math. Soc. 7 (1994), 335-381; J. Amer. Math. Soc. 7 (1994), 383-453 (1993). MR1186962 (93m:17014). MR.1239506 (94g:17048) MR:1239507 (94g:17049)

[KT95] Masaki Kashiwara and Toshiyuki Tanisaki, Kazhdan-Lusztig conjecture for affine Lie algebras with negative level, Duke Math. J. 77 (1995), no. 1, 21-62. MR.1317626 (96j:17016)

[Kum02] Shrawan Kumar, Kac-Moody groups, their flag varieties and representation theory, Progress in Mathematics, vol. 204, Birkhäuser Boston Inc., Boston, MA, 2002. MR.1923198 (2003k:22022)

[Lus80a] George Lusztig, Hecke algebras and Jantzen's generic decomposition patterns, Adv. in Math. 37 (1980), no. 2, 121-164. MR.591724 (82b:20059)

[Lus80b] _ Some problems in the representation theory of finite Chevalley groups, The Santa Cruz Conference on Finite Groups (Univ. California, Santa Cruz, Calif., 1979), Proc. Sympos. Pure Math., vol. 37, Amer. Math. Soc., Providence, R.I., 1980, pp. 313317. MR604598 (82i:20014)

[Lus90] , Quantum groups at roots of 1, Geometria Dedicata 35 (1990), 89-114. MR.1066560 (91j:17018)

[Soe90] Wolfgang Soergel, Kategorie $\mathcal{O}$, perverse Garben und Moduln über den Koinvarianten zur Weylgruppe, J. Amer. Math. Soc. 3 (1990), No.2, 421-445. MR.1029692(91e:17007)

[Soe95] _ Roots of unity and positive characteristic, Representations of groups (Banff, AB, 1994), CMS Conf. Proc., vol. 16, Amer. Math. Soc., Providence, RI, 1995, pp. 315338. MR1357204 (96i:20057)

[Soe97] _ Kazhdan-Lusztig-Polynome und eine Kombinatorik für Kipp-Moduln, Represent. Theory 1 (1997), 37-68 (electronic). MR.1445511 (99d:17023)

[Soe00] - On the relation between intersection cohomology and representation theory in positive characteristic, J. Pure Appl. Algebra 152 (2000), no. 1-3, 311-335, Commutative algebra, homological algebra and representation theory (Catania/Genoa/Rome, 1998). MR1784005 (2001k:20098)

Department Mathematik, Universität Erlangen-Nürnberg, Bismarckstr. $1 \frac{1}{2}, 91054$ Erlangen, Germany

E-mail address: fiebig@mi.uni-erlangen.de 